\title{
Pricing and Resource Allocation via Game Theory for a Small-Cell Video Caching System
}

\author{
Jun Li, Member, IEEE, He Chen, Member, IEEE, Youjia Chen, Zihuai Lin, Senior Member, IEEE, \\ Branka Vucetic, Fellow, IEEE, and Lajos Hanzo, Fellow, IEEE
}

\begin{abstract}
Evidence indicates that downloading on-demand videos accounts for a dramatic increase in data traffic over cellular networks. Caching popular videos in the storage of smallcell base stations (SBS), namely, small-cell caching, is an efficient technology for reducing the transmission latency while mitigating the redundant transmissions of popular videos over back-haul channels. In this paper, we consider a commercialized small-cell caching system consisting of a network service provider (NSP), several video retailers (VRs), and mobile users (MUs). The NSP leases its SBSs to the VRs for the purpose of making profits, and the VRs, after storing popular videos in the rented SBSs, can provide faster local video transmissions to the MUs, thereby gaining more profits. We conceive this system within the framework of Stackelberg game by treating the SBSs as specific types of resources. We first model the MUs and SBSs as two independent Poisson point processes, and develop, via stochastic geometry theory, the probability of the specific event that an MU obtains the video of its choice directly from the memory of an SBS. Then, based on the probability derived, we formulate a Stackelberg game to jointly maximize the average profit of both the NSP and the VRs. In addition, we investigate the Stackelberg equilibrium by solving a non-convex optimization problem. With the aid of this game theoretic framework, we shed light on the relationship between four important factors: the optimal pricing of leasing an SBS, the SBSs allocation among the VRs, the storage size of the SBSs, and the popularity distribution of the VRs. Monte Carlo simulations show that our stochastic geometry-based analytical results closely match the empirical ones. Numerical results are also provided for quantifying the proposed game-theoretic framework by showing its efficiency on pricing and resource allocation.
\end{abstract}

Manuscript received May 28, 2015; revised November 30, 2015; accepted February 16, 2016. This work was supported in part by the National Natural Science Foundation of China under Grant 61501238, Grant 61271230, and Grant 61472190, in part by the Jiangsu Provincial Science Foundation under Project BK20150786, in part by the Specially Appointed Professor Program in Jiangsu Province, 2015, in part by the Open Research Fund of National Key Laboratory of Electromagnetic Environment under Grant 201500013 , in part by the Open Research Fund of National Mobile Communications Research Laboratory, Southeast University, under Grant 2013D02, in part by the Australian Research Council under Grant DP120100405 and Grant DP150104019, and in part by the Faculty of Engineering and IT Early Career Researcher Scheme 2016, The University of Sydney. (Corresponding author: Jun Li.)

$\mathrm{J} . \mathrm{Li}$ is with the School of Electronic and Optical Engineering, Nanjing University of Science and Technology, Nanjing 210094, China (e-mail: jun.li@njust.edu.cn).

H. Chen, Y. Chen, Z. Lin, and B. Vucetic are with the School of Electrical and Information Engineering, The University of Sydney, Sydney, NSW 2006 Australia (e-mail: he.chen@sydney.edu.au; youjia.chen@sydney.edu.au; linzihuai@ieee.org; branka.vucetic@sydney.edu.au).

L. Hanzo is with the Department of Electronics and Computer Science, University of Southampton, Southampton SO17 1BJ, U.K. (e-mail: lh@ecs.soton.ac.uk).

Color versions of one or more of the figures in this paper are available online at http://ieeexplore.ieee.org.

Digital Object Identifier 10.1109/JSAC.2016.2577278
Index Terms-Small-cell caching, cellular networks, stochastic geometry, Stackelberg game.

\section{INTRODUCTION}

W IRELESS data traffic is expected to increase exponentially in the next few years driven by a staggering proliferation of mobile users (MU) and their bandwidthhungry mobile applications. There is evidence that streaming of on-demand videos by the MUs is the major reason for boosting the tele-traffic over cellular networks [1]. According to the prediction of mobile data traffic by Cisco, mobile video streaming will account for $72 \%$ of the overall mobile data traffic by 2019. The on-demand video downloading involves repeated wireless transmission of videos that are requested multiple times by different users in a completely asynchronous manner, which is different from the transmission style of live video streaming.

Often, there are numerous repetitive requests of popular videos from the MUs, such as online blockbusters, leading to redundant video transmissions. The redundancy of data transmissions can be reduced by locally storing popular videos, known as caching, into the storage of intermediate network nodes, effectively forming a local caching system [1], [2]. The local caching brings video content closer to the MUs and alleviates redundant data transmissions via redirecting the downloading requests to the intermediate nodes.

Generally, wireless data caching consists of two stages: data placement and data delivery [3]. In the data placement stage, popular videos are cached into local storages during off-peak periods, while during the data delivery stage, videos requested are delivered from the local caching system to the MUs. Recent works advanced the caching solutions of both device-to-device (D2D) networks and wireless sensor networks [4]-[6]. Specifically, in [4] a caching scheme was proposed for a D2D based cellular network relaying on the MUs' caching of popular video content. In this scheme, the D2D cluster size was optimized for reducing the downloading delay. In [5] and [6], the authors proposed novel caching schemes for wireless sensor networks, where the protocol model of [7] was adopted.

Since small-cell embedded architectures will dominate in future cellular networks, known as heterogeneous networks (HetNet) [8]-[13], caching relying on small-cell base stations (SBS), namely, small-cell caching, constitutes a promising solution for HetNets. The advantages brought about by small-cell caching are threefold. Firstly, popular videos are placed closer to the MUs when they are cached in SBSs, hence 
reducing the transmission latency. Secondly, redundant transmissions over SBSs' back-haul channels, which are usually expensive [14], can be mitigated. Thirdly, the majority of video traffic is offloaded from macro-cell base stations to SBSs.

In [15], a small-cell caching scheme, named 'Femtocaching', is proposed for a cellular network having embedded SBSs, where the data placement at the SBSs is optimized in a centralized manner for the sake of reducing the transmission delay imposed. However, [15] considers an idealized system, where neither the interference nor the impact of wireless channels is taken into account. The associations between the MUs and the SBSs are pre-determined without considering the specific channel conditions encountered. In [16], small-cell caching is investigated in the context of stochastic networks. The average performance is quantified with the aid of stochastic geometry [17], [18], where the distribution of network nodes is modeled by Poisson point process (PPP). However, the caching strategy of [16] assumes that the SBSs cache the same content, hence leading to a sub-optimal solution.

As detailed above, current research on wireless caching mainly considers the data placement issue optimized for reducing the downloading delay. However, the entire caching system design involves numerous issues apart from data placement. From a commercial perspective, it will be more interesting to consider the topics of pricing for video streaming, the rental of local storage, and so on. A commercialized caching system may consist of video retailers (VR), network service providers (NSP) and MUs. The VRs, e.g., Youtube, purchase copyrights from video producers and publish the videos on their web-sites. The NSPs are typically operators of cellular networks, who are in charge of network facilities, such as macro-cell base stations and SBSs.

In such a commercial small-cell caching system, the VRs' revenue is acquired from providing video streaming for the MUs. As the central servers of the VRs, which store the popular videos, are usually located in the backbone networks and far away from the MUs, an efficient solution is to locally cache these videos, thereby gaining more profits from providing faster local transmissions. In turn, these local caching demands raised by the VRs offer the NSPs profitable opportunities from leasing their SBSs. Additionally, the NSPs can save considerable costs due to reduced redundant video transmissions over SBSs' back-haul channels. In this sense, both the VRs and NSPs are the beneficiaries of the local caching system. However, each entity is selfish and wishes to maximize its own benefit, raising a competition and optimization problem among these entities, which can be effectively solved within the framework of game theory.

We note that game theory has been successfully applied to wireless communications for solving resource allocation problems. In [19], the authors propose a dynamic spectrum leasing mechanism via power control games. In [20], a price-based power allocation scheme is proposed for spectrum sharing in Femto-cell networks based on Stackelberg game. Game theoretical power control strategies for maximizing the utility in spectrum sharing networks are studied in [21] and [22].
In this paper, we propose a commercial small-cell caching system consisting of an NSP, multiple VRs and MUs. We optimize such a system within the framework of Stackelberg game by viewing the SBSs as a specific type of resources for the purpose of video caching. Generally speaking, Stackelberg game is a strategic game that consists of a leader and several followers competing with each other for certain resources [23]. The leader moves first and the followers move subsequently. Correspondingly, in our game theoretic caching system, we consider the NSP to be the leader and the VRs as the followers. The NSP sets the price of leasing an SBS, while the VRs compete with each other for renting a fraction of the SBSs.

To the best of the authors' knowledge, our work is the first of its kind that optimizes a caching system with the aid of game theory. Compared to many other game theory based resource allocation schemes, where the power, bandwidth and time slots are treated as the resources, our work has a totally different profit model, established based on our coverage derivations. In particular, our contributions are as follows.

1) By following the stochastic geometry framework of [17] and [18], we model the MUs and SBSs in the network as two different ties of a Poisson point process (PPP) [24]. Under this network model, we define the concept of a successful video downloading event when an MU obtains the requested video directly from the storage of an SBS. Then we quantify the probability of this event based on stochastic geometry theory.

2) Based on the probability derived, we develop a profit model of our caching system and formulate the profits gained by the NSP and the VRs from SBSs leasing and renting.

3) A Stackelberg game is proposed for jointly maximizing the average profit of the NSP and the VRs. Given this game theoretic framework, we investigate a non-uniform pricing scheme, where the price charged to different VRs varies.

4) Then we investigate the Stackelberg equilibrium of this scheme via solving a non-convex optimization problem. It is interesting to observe that the optimal solution is related both to the storage size of each SBS and to the popularity distribution of the VRs.

5) Furthermore, we consider an uniform pricing scheme. We find that although the uniform pricing scheme is inferior to the non-uniform one in terms of maximizing the NSP's profit, it is capable of reducing more backhaul costs compared with the latter and achieves the maximum sum profit of the NSP and the VRs.

The rest of this paper is organized as follows. We describe the system model in Section II and establish the related profit model in Section III. We then formulate Stackelberg game for our small-cell caching system in Section IV. In Section V, we investigate Stackelberg equilibrium for the non-uniform pricing scheme by solving a non-convex optimization problem, while in Section VI, we further consider the uniform pricing scheme. Our simulations and numerical results are detailed in Section VII, while our conclusions are provided in Section VIII. 


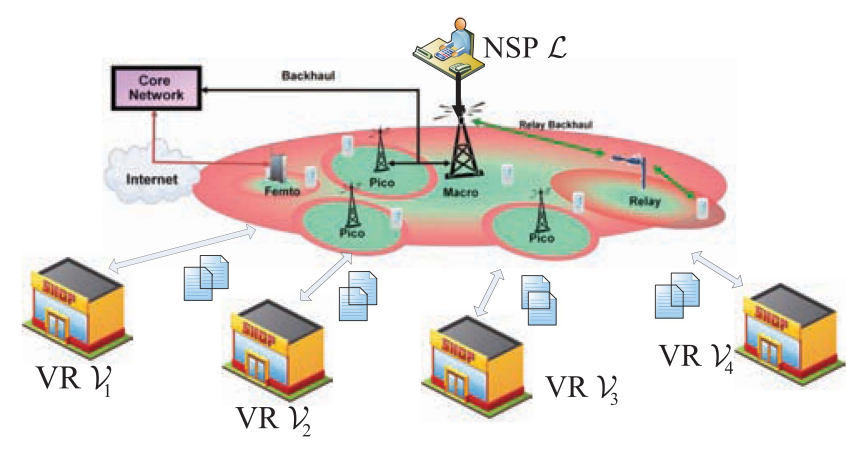

Fig. 1. An example of the small-cell caching system with four VRs.

\section{SYSTEM MODEL}

We consider a commercial small-cell caching system consisting of an NSP, $V$ VRs, and a number of MUs. Let us denote by $\mathcal{L}$ the NSP, by $\mathcal{V}=\left\{\mathcal{V}_{1}, \mathcal{V}_{2}, \cdots, \mathcal{V}_{V}\right\}$ the set of the VRs, and by $\mathcal{M}$ one of the MUs. Fig. 1 shows an example of our caching system relying on four VRs. In such a system, the VRs wish to rent the SBSs from $\mathcal{L}$ for placing their videos. Both the NSP and each VR aim for maximizing their profits.

There are three stages in our system. In the first stage, the VRs purchase the copyrights of popular videos from video producers and publish them on their web-sites. In the second stage, the VRs negotiate with the NSP on the rent of SBSs for caching these popular videos. In the third stage, the MUs connect to the SBSs for downloading the desired videos. We will particulary focus our attention on the second and third stages within this game theoretic framework.

\section{A. Network Model}

Let us consider a small-cell based caching network composed of the MUs and the SBSs owned by $\mathcal{L}$, where each SBS is deployed with a fixed transmit power $P$ and the storage of $Q$ video files. Let us assume that the SBSs transmit over the channels that are orthogonal to those of the macro-cell base stations, and thus there is no interference incurred by the macro-cell base stations. Also, assume that these SBSs are spatially distributed according to a homogeneous PPP (HPPP) $\Phi$ of intensity $\lambda$. Here, the intensity $\lambda$ represents the number of the SBSs per unit area. Furthermore, we model the distribution of the MUs as an independent HPPP $\Psi$ of intensity $\zeta$.

The wireless down-link channels spanning from the SBSs to the MUs are independent and identically distributed (i.i.d.), and modeled as the combination of path-loss and Rayleigh fading. Without loss of generality, we carry out our analysis for a typical MU located at the origin. The path-loss between an SBS located at $x$ and the typical MU is denoted by $\|x\|^{-\alpha}$, where $\alpha$ is the path-loss exponent. The channel power of the Rayleigh fading between them is denoted by $h_{x}$, where $h_{x} \sim \exp (1)$. The noise at an MU is Gaussian distributed with a variance $\sigma^{2}$.

We consider the steady-state of a saturated network, where all the SBSs keep on transmitting data in the entire frequency band allocated. This modeling approach for saturated networks characterizes the worst-case scenario of the real systems, which has been adopted by numerous studies on PPP analysis, such as [18]. Hence, the received signal-to-interference-plusnoise ratio (SINR) at the typical MU from an SBS located at $x$ can be expressed as

$$
\rho(x)=\frac{P h_{x}\|x\|^{-\alpha}}{\sum_{x^{\prime} \in \Phi \backslash x} P h_{x^{\prime}}\left\|x^{\prime}\right\|^{-\alpha}+\sigma^{2}} .
$$

The typical MU is considered to be "covered" by an SBS located at $x$ as long as $\rho(x)$ is no lower than a pre-set SINR threshold $\delta$, i.e.,

$$
\rho(x) \geq \delta .
$$

Generally, an MU can be covered by multiple SBSs. Note that the SINR threshold $\delta$ defines the highest delay of downloading a video file. Since the quality and code rate of a video clip have been specified within the video file, the download delay will be the major factor predetermining the QoS perceived by the mobile users. Therefore, we focus our attention on the coverage and SINR in the following derivations.

\section{B. Popularity and Preferences}

We now model the popularity distribution, i.e., the distribution of request probabilities, among the popular videos to be cached. Let us denote by $\mathcal{F}=\left\{\mathcal{F}_{1}, \mathcal{F}_{2}, \cdots, \mathcal{F}_{N}\right\}$ the file set consisting of $N$ video files, where each video file contains an individual movie or video clip that is frequently requested by MUs. The popularity distribution of $\mathcal{F}$ is represented by a vector $\mathbf{t}=\left[t_{1}, t_{2}, \cdots, t_{N}\right]$. That is, the MUs make independent requests of the $n$-th video $\mathcal{F}_{n}, n=1, \cdots, N$, with the probability of $t_{n}$. Generally, $\mathbf{t}$ can be modeled by the Zipf distribution [25] as

$$
t_{n}=\frac{1 / n^{\beta}}{\sum_{j=1}^{N} 1 / j^{\beta}}, \quad \forall n,
$$

where the exponent $\beta$ is a positive value, characterizing the video popularity. A higher $\beta$ corresponds to a higher content reuse, where the most popular files account for the majority of download requests. From Eq. (3), the file with a smaller $n$ corresponds to a higher popularity.

Note that each SBS can cache at most $Q$ video files, and usually $Q$ is no higher than the number of videos in $\mathcal{F}$, i.e., we have $Q \leq N$. Without loss of generality, we assume that $N / Q$ is an integer. The $N$ files in $\mathcal{F}$ are divided into $F=N / Q$ file groups (FG), with each FG containing $Q$ video files. The $n$-th video, $\forall n \in\{(f-1) Q+1, \cdots, f Q\}$, is included in the $f$-th FG, $f=1, \cdots, F$. Denote by $\mathcal{G}_{f}$ the $f$-th FG, and by $p_{f}$ the probability of the MUs' requesting a file in $\mathcal{G}_{f}$, and we have

$$
p_{f}=\sum_{n=(f-1) Q+1}^{f Q} t_{n}, \quad \forall f .
$$

File caching is then carried out on the basis of FGs, where each SBS caches one of the $F$ FGs.

At the same time, the MUs have unbalanced preferences with regard to the $V$ VRs, i.e., some VRs are more popular than others. For example, the majority of the MUs may tend to access Youtube for video streaming. The preference distribution among the VRs is denoted by $\mathbf{q}=\left[q_{1}, q_{2}, \cdots, q_{V}\right]$, 
where $q_{v}, v=1, \cdots, V$, represents the probability that the MUs prefer to download videos from $\mathcal{V}_{v}$. The preference distribution $\mathbf{q}$ can also be modeled by the Zipf distribution. Hence, we have

$$
q_{v}=\frac{1 / v^{\gamma}}{\sum_{j=1}^{V} 1 / j^{\gamma}}, \quad \forall v,
$$

where $\gamma$ is a positive value, characterizing the preference of the VRs. A higher $\gamma$ corresponds to a higher probability of accessing the most popular VRs.

\section{Video Placement and Download}

Next, we introduce the small-cell caching system with its detailed parameters. In the first stage, each VR purchases the $N$ popular videos in $\mathcal{F}$ from the producers and publishes these videos on its web-site. In the second stage, upon obtaining these videos, the VRs negotiate with the NSP $\mathcal{L}$ for renting its SBSs. As $\mathcal{L}$ leases its SBSs to multiple VRs, we denote by $\boldsymbol{\tau}=\left[\tau_{1}, \tau_{2}, \cdots, \tau_{V}\right]$ the fraction vector, where $\tau_{v}$ represents the fraction of the SBSs that are assigned to $\mathcal{V}_{v}, \forall v$. We assume that the SBSs rented by each VR are uniformly distributed. Hence, the SBSs that are allocated to $\mathcal{V}_{v}$ can be modeled as a "thinned" HPPP $\Phi_{v}$ with intensity $\tau_{v} \lambda$.

The data placements of the second stage commence during network off-peak time after the VRs obtain access to the SBSs. During the placements, each SBS will be allocated with one of the $F$ FGs. Generally, we assume that the VRs do not have the a priori information regarding the popularity distribution of $\mathcal{F}$. This is because the popularity of videos is changing periodically, and can only be obtained statistically after these videos quit the market. It is clear that each VR may have more or less some statistical information on the popularity distribution of videos based on the MUs' downloading history. However, this information will be biased due to limited sampling. In this case, the VRs will uniformly assign the $F$ FGs to the SBSs with equal probability of $\frac{1}{F}$ for simplicity. We are interested in investigating the uniform assignment of video files for drawing a bottom line of the system performance. As the FGs are randomly assigned, the SBSs in $\Phi_{v}$ that cache the FG $\mathcal{G}_{f}$ can be further modeled as a "more thinned" HPPP $\Phi_{v, f}$ with an intensity of $\frac{1}{F} \tau_{v} \lambda$.

In the third stage, the MUs start to download videos. When an MU $\mathcal{M}$ requires a video of $\mathcal{G}_{f}$ from $\mathcal{V}_{v}$, it searches the SBSs in $\Phi_{v, f}$ and tries to connect to the nearest SBS that covers $\mathcal{M}$. Provided that such an SBS exists, the MU $\mathcal{M}$ will obtain this video directly from this SBS, and we thereby define this event by $\mathcal{E}_{v, f}$. By contrast, if such an SBS does not exist, $\mathcal{M}$ will be redirected to the central servers of $\mathcal{V}_{v}$ for downloading the requested file. Since the servers of $\mathcal{V}_{v}$ are located at the backbone network, this redirection of the demand will trigger a transmission via the back-haul channels of the NSP $\mathcal{L}$, hence leading to an extra cost.

\section{Profit Modeling}

We now focus on modeling the profit of the NSP and the VRs obtained from the small-cell caching system. The average profit is developed based on stochastically geometrical distributions of the network nodes in terms of per unit area times unit period $(/ U A P)$, e.g., /month $\cdot \mathrm{km}^{2}$.

\section{A. Average Profit of the NSP}

For the NSP $\mathcal{L}$, the revenue gained from the caching system consists of two parts: 1) the income gleaned from leasing SBSs to the VRs and 2) the cost reduction due to reduced usage of the SBSs' back-haul channels. First, the leasing income/U AP of $\mathcal{L}$ can be calculated as

$$
S^{R T}=\sum_{j=1}^{V} \tau_{j} \lambda s_{j},
$$

where $s_{j}$ is the price per unit period charged to $\mathcal{V}_{j}$ for renting an SBS. Then we formulate the saved cost/UAP due to reduced back-haul channel transmissions. When an MU demands a video in $\mathcal{G}_{f}$ from $\mathcal{V}_{v}$, we derive the probability $\operatorname{Pr}\left(\mathcal{E}_{v, f}\right)$ as follows.

Theorem 1: The probability of the event $\mathcal{E}_{v, f}, \forall v, f$, can be expressed as

$$
\operatorname{Pr}\left(\mathcal{E}_{v, f}\right)=\frac{\tau_{v}}{C(\delta, \alpha)\left(F-\tau_{v}\right)+A(\delta, \alpha) \tau_{v}+\tau_{v}},
$$

where we have $A(\delta, \alpha) \triangleq \frac{2 \delta}{\alpha-2} \quad{ }_{2} F_{1}\left(1,1-\frac{2}{\alpha} ; 2-\frac{2}{\alpha} ;-\delta\right)$ and $C(\delta, \alpha) \triangleq \frac{2}{\alpha} \delta^{\frac{2}{\alpha}} B\left(\frac{2}{\alpha}, 1-\frac{2}{\alpha}\right)$. Furthermore, ${ }_{2} F_{1}(\cdot)$ in the function $A(\delta, \alpha)$ is the hypergeometric function, while the Beta function in $C(\delta, \alpha)$ is formulated as $B(x, y)=$ $\int_{0}^{1} t^{x-1}(1-t)^{y-1} \mathrm{~d} t$

Proof: Please refer to Appendix A.

Remark 1: From Theorem 1, it is interesting to observe that the probability $\operatorname{Pr}\left(\mathcal{E}_{v, f}\right)$ is independent of both the transmit power $P$ and the intensity $\lambda$ of the SBSs. Furthermore, since $Q$ is inversely proportional to $F$, we can enhance $\operatorname{Pr}\left(\mathcal{E}_{v, f}\right)$ by increasing the storage size $Q$.

We assume that there are on average $K$ video requests from each MU within unit period, and that the average back-haul cost for a video transmission is $s^{b h}$. Based on $\operatorname{Pr}\left(\mathcal{E}_{v, f}\right)$ in Eq. (7), we obtain the cost reduction/UAP for the back-haul channels of $\mathcal{L}$ as

$$
S^{B H}=\sum_{j_{1}=1}^{F} \sum_{j_{2}=1}^{V} p_{j_{1}} q_{j_{2}} \zeta K \operatorname{Pr}\left(\mathcal{E}_{j_{2}, j_{1}}\right) s^{b h} .
$$

By combining the above two items, the overall profit/UAP for $\mathcal{L}$ can be expressed as

$$
S^{N S P}=S^{R T}+S^{B H} .
$$

\section{B. Average Profit of the VRs}

Note that the MUs can download the videos either from the memories of the SBSs directly or from the servers of the VRs at backbone networks via back-haul channels. In the first case, the MUs will be levied by the VRs an extra amount of money in addition to the videos' prices because of the higher-rate local streaming, namely, local downloading surcharge (LDS). We assume that the LDS of each video is set as $s^{l d}$. Then the revenue $/ U A P$ for a VR $V_{v}$ gained from the LDS can be calculated as

$$
S_{v}^{L D}=\sum_{j=1}^{F} p_{j} q_{v} \zeta K \operatorname{Pr}\left(\mathcal{E}_{v, j}\right) s^{l d} .
$$


Additionally, $\mathcal{V}_{v}$ pays for renting the SBSs from $\mathcal{L}$. The related cost/UAP can be written as

$$
S_{v}^{R T}=\tau_{v} \lambda s_{v} .
$$

Upon combining the two items, the profit/UAP for $\mathcal{V}_{v}, \forall v$, can be expressed as

$$
S_{v}^{V R}=S_{v}^{L D}-S_{v}^{R T} .
$$

\section{Problem Formulation}

In this section, we first present the Stackelberg game formulation for our price-based SBS allocation scheme. Then the equilibrium of the proposed game is investigated.

\section{A. Stackelberg Game Formulation}

Again, Stackelberg game is a strategic game that consists of a leader and several followers competing with each other for certain resources [23]. The leader moves first and the followers move subsequently. In our small-cell caching system, we model the NSP $\mathcal{L}$ as the leader, and the $V$ VRs as the followers. The NSP imposes a price vector $\mathbf{s}=\left[s_{1}, s_{2}, \cdots, s_{V}\right]$ for the lease of its SBSs, where $s_{v}, \forall v$, has been defined in the previous section as the price per unit period charged on $\mathcal{V}_{v}$ for renting an SBS. After the price vector $\mathbf{s}$ is set, the VRs update the fraction $\tau_{v}, \forall v$, that they tend to rent from $\mathcal{L}$.

1) Optimization Formulation of the Leader: Observe from the above game model that the NSP's objective is to maximize its profit $S^{N S P}$ formulated in Eq. (9). Note that for $\forall v$, the fraction $\tau_{v}$ is a function of the price $s_{v}$ under the Stackelberg game formulation. This means that the fraction of the SBSs that each VR is willing to rent depends on the specific price charged to them for renting an SBS. Consequently, the NSP has to find the optimal price vector $\mathbf{s}$ for maximizing its profit. This optimization problem can be summarized as follows.

Problem 1: The optimization problem of maximizing $\mathcal{L}$ 's profit can be formulated as

$$
\begin{aligned}
& \max _{\mathbf{s} \geq \mathbf{0}} S^{N S P}(\mathbf{s}, \boldsymbol{\tau}), \\
& \text { s.t. } \sum_{j=1}^{V} \tau_{j} \leq 1 .
\end{aligned}
$$

2) Optimization Formulation of the Followers: The profit gained by the VR $\mathcal{V}_{v}$ in Eq. (12) can be further written as

$$
\begin{aligned}
S_{v}^{V R}\left(\tau_{v}, s_{v}\right)= & \sum_{j=1}^{F} p_{j} q_{v} \zeta K \operatorname{Pr}\left(\mathcal{E}_{v, j}\right) s^{l d}-\tau_{v} \lambda s_{v} \\
= & \sum_{j=1}^{F} \frac{p_{j} q_{v} \zeta K s^{l d} \tau_{v}}{(A(\delta, \alpha)-C(\delta, \alpha)+1) \tau_{v}+C(\delta, \alpha) F} \\
& -\lambda s_{v} \tau_{v} .
\end{aligned}
$$

We can see from Eq. (14) that once the price $s_{v}$ is fixed, the profit of $\mathcal{V}_{v}$ depends on $\tau_{v}$, i.e., the fraction of SBSs that are rented by $\mathcal{V}_{v}$. If $\mathcal{V}_{v}$ increases the fraction $\tau_{v}$, it will gain more revenue by levying surcharges from more MUs, while at the same time, $\mathcal{V}_{v}$ will have to pay for renting more SBSs.
Therefore, $\tau_{v}$ has to be optimized for maximizing the profit of $\mathcal{V}_{v}$. This optimization can be formulated as follows.

Problem 2: The optimization problem of maximizing $\mathcal{V}_{v}$ 's profit can be written as

$$
\max _{\tau_{v} \geq 0} S_{v}^{V R}\left(\tau_{v}, s_{v}\right) .
$$

Problem 1 and Problem 2 together form a Stackelberg game. The objective of this game is to find the Stackelberg Equilibrium (SE) points from which neither the leader (NSP) nor the followers (VRs) have incentives to deviate. In the following, we investigate the SE points for the proposed game.

\section{B. Stackelberg Equilibrium}

For our Stackelberg game, the SE is defined as follows.

Definition 1: Let $\mathbf{s}^{\star} \triangleq\left[s_{1}^{\star}, s_{2}^{\star}, \cdots, s_{V}^{\star}\right]$ be a solution for Problem 1, and $\tau_{v}^{\star}$ be a solution for Problem 2, $\forall v$. Define $\boldsymbol{\tau}^{\star} \triangleq\left[\tau_{1}^{\star}, \tau_{2}^{\star}, \cdots, \tau_{V}^{\star}\right]$. Then the point $\left(\mathbf{s}^{\star}, \boldsymbol{\tau}^{\star}\right)$ is an SE for the proposed Stackelberg game if for any $(\mathbf{s}, \boldsymbol{\tau})$ with $\mathbf{s} \succeq \mathbf{0}$ and $\boldsymbol{\tau} \succeq \mathbf{0}$, the following conditions are satisfied:

$$
\begin{aligned}
S^{N S P}\left(\mathbf{s}^{\star}, \boldsymbol{\tau}^{\star}\right) & \geq S^{N S P}\left(\mathbf{s}, \boldsymbol{\tau}^{\star}\right), \\
S_{v}^{V R}\left(s_{v}^{\star}, \tau_{v}^{\star}\right) & \geq S_{v}^{V R}\left(s_{v}^{\star}, \tau_{v}\right), \quad \forall v .
\end{aligned}
$$

Generally speaking, the SE of a Stackelberg game can be obtained by finding its perfect Nash Equilibrium (NE). In our proposed game, we can see that the VRs strictly compete in a non-cooperative fashion. Therefore, a non-cooperative subgame on controlling the fractions of rented SBSs is formulated at the VRs' side. For a non-cooperative game, the $\mathrm{NE}$ is defined as the operating points at which no players can improve utility by changing its strategy unilaterally. At the NSP's side, since there is only one player, the best response of the NSP is to solve Problem 1. To achieve this, we need to first find the best response functions of the followers, based on which, we solve the best response function for the leader.

Therefore, in our game, we first solve Problem 2 given a price vector $\mathbf{s}$. Then with the obtained best response function $\boldsymbol{\tau}^{\star}$ of the VRs, we solve Problem 1 for the optimal price $\mathbf{s}^{\star}$. In the following, we will have an in-depth investigation on this game theoretic optimization.

\section{GAME Theoretic Optimization}

In this section, we will solve the optimization problem in our game under the non-uniform pricing scheme, where the NSP $\mathcal{L}$ charges the VRs with different prices $s_{1}, \cdots, s_{V}$ for renting an SBS. In this scheme, we first solve Problem 2 at the VRs, and rewrite Eq. (14) as

$$
S_{v}^{V R}\left(\tau_{v}, s_{v}\right)=\frac{\Gamma_{v} s^{l d} \tau_{v}}{\Theta \tau_{v}+\Lambda}-\lambda s_{v} \tau_{v} .0
$$

where $\Gamma_{v} \triangleq \sum_{j=1}^{F} p_{j} q_{v} \zeta K, \Theta \triangleq A(\delta, \alpha)-C(\delta, \alpha)+1$, and $\Lambda \triangleq C(\delta, \alpha) F$. We observe that Eq. (17) is a concave function over the variable $\tau_{v}$. Thus, we can obtain the optimal solution by solving the Karush-Kuhn-Tucker (KKT) conditions, and we have the following lemma. 
Lemma 1: For a given price $s_{v}$, the optimal solution of Problem 2 is

$$
\tau_{v}^{\star}=\left(\sqrt{\frac{\Gamma_{v} \Lambda s^{l d}}{\Theta^{2} \lambda}} \sqrt{\frac{1}{s_{v}}}-\frac{\Lambda}{\Theta}\right)^{+},
$$

where $(\cdot)^{+} \triangleq \max (\cdot, 0)$.

Proof: The optimal solution $\tau_{v}^{\star}$ of $\mathcal{V}_{v}$ can be obtained by deriving $S_{v}^{V R}$ with respect to $\tau_{v}$ and solving $\frac{\mathrm{d} S_{v}^{V R}}{\mathrm{~d} \tau_{v}}=0$ under the constraint that $\tau_{v} \geq 0$.

We can see from Lemma 1 that if the price $s_{v}$ is set too high, i.e., $s_{v} \geq \frac{\Gamma_{v} s^{l d}}{\Lambda \lambda}$, the VR $\mathcal{V}_{v}$ will opt out for renting any SBS from $\mathcal{L}$ due the high price charged. Consequently, the VR $\mathcal{V}_{v}$ will not participate in the game.

In the following derivations, we assume that the LDS on each video $s^{l d}$ is set by the VRs to be the cost of a video transmission via back-haul channels $s^{b h}$. The rational behind this assumption is as follows. Since a local downloading reduce a back-haul transmission, this saved back-haul transmission can be potentially utilized to provide extra services (equivalent to the value of $s^{b h}$ ) for the MUs. In addition, the MUs enjoy the benefit from faster local video transmissions. In light of this, it is reasonable to assume that the MUs are willing to accept the price $s^{b h}$ for a local video transmission.

Substituting the optimal $\tau_{v}^{\star}$ of Eq. (18) into Eq. (9) and carry out some further manipulations, we arrive at

$$
\begin{array}{r}
S^{N S P}=\sum_{j=1}^{V} \lambda s_{j}\left(\sqrt{\frac{\Gamma_{j} \Lambda s^{b h}}{\Theta^{2} \lambda}} \sqrt{\frac{1}{s_{j}}}-\frac{\Lambda}{\Theta}\right)^{+} \\
+\frac{\sum_{i=1}^{F} p_{i} q_{j} \zeta K s^{b h}\left(\sqrt{\frac{\Gamma_{j} \Lambda s^{b h}}{\Theta^{2} \lambda}} \sqrt{\frac{1}{s_{j}}}-\frac{\Lambda}{\Theta}\right)^{+}}{\Theta\left(\sqrt{\frac{\Gamma_{j} \Lambda s^{b h}}{\Theta^{2} \lambda}} \sqrt{\frac{1}{s_{j}}}-\frac{\Lambda}{\Theta}\right)^{+}+\Lambda}
\end{array}
$$

$=\sum_{j=1}^{V} \frac{\xi_{i}}{\Theta}\left(-\Lambda \lambda s_{j}+\left(\sqrt{s^{b h}}-\frac{s^{b h}}{\sqrt{s^{b h}}}\right) \sqrt{\Gamma_{j} \Lambda \lambda s_{j}}+\Gamma_{j} s^{b h}\right)$

$=\sum_{j=1}^{V} \frac{\xi_{i}}{\Theta}\left(-\Lambda \lambda s_{j}+\Gamma_{j} s^{b h}\right)$,

where $\xi_{j}$ is the indicator function, with $\xi_{j}=1$ if $s_{j}<\frac{\Gamma_{j} s^{b h}}{\Lambda \lambda}$ and $\xi_{j}=0$ otherwise. Upon defining the binary vector $\xi \triangleq$ $\left[\xi_{1}, \xi_{2}, \cdots, \xi_{V}\right]$, we can rewrite Problem 1 as follows.

Problem 3: Given the optimal solutions $\tau_{v}^{\star}, \forall v$, gleaned from the followers, we can rewrite Problem 1 as

$$
\begin{aligned}
& \min _{\boldsymbol{\xi}, \mathbf{s} \geq \mathbf{0}} \sum_{j=1}^{V} \xi_{j}\left(\Lambda \lambda s_{j}-\Gamma_{j} s^{b h}\right), \\
& \text { s.t. } \sum_{j=1}^{V} \xi_{j}\left(\sqrt{\frac{\Gamma_{j} \Lambda s^{b h}}{\lambda s_{j}}}-\Lambda\right) \leq \Theta .
\end{aligned}
$$

Observe from Eq. (20) that Problem 3 is non-convex due to $\xi$. However, for a given $\xi$, this problem can be solved by satisfying the KKT conditions. In the following, we commence with the assumption that $\boldsymbol{\xi}=\mathbf{1}$, i.e., $\xi_{v}=1, \forall v$, and then we extend this result to the general case.

\section{A. Special Case: $\xi_{v}=1, \forall v$}

In this case, all the VRs are participating in the game, and we have the following optimization problem.

Problem 4: Assuming $\xi_{v}=1, \forall v$, we rewrite Problem 3 as

$$
\begin{aligned}
& \min _{\mathbf{s} \geq \mathbf{0}} \sum_{j=1}^{V} s_{j}, \\
& \text { s.t. } \sum_{j=1}^{V} \sqrt{\frac{\Gamma_{j}}{s_{j}}} \leq(V \Lambda+\Theta) \sqrt{\frac{\lambda}{\Lambda s^{b h}}} .
\end{aligned}
$$

The optimal solution of Problem 4 is derived and given in the following lemma.

Lemma 2: The optimal solution to Problem 4 can be derived as $\hat{\mathbf{s}} \triangleq\left[\hat{s}_{1}, \cdots, \hat{s}_{V}\right]$, where

$$
\hat{s}_{v}=\frac{\Lambda s^{b h}\left(\sum_{j=1}^{V} \sqrt[3]{\Gamma_{j}}\right)^{2} \sqrt[3]{\Gamma_{v}}}{\lambda(V \Lambda+\Theta)^{2}}, \quad \forall v .
$$

Proof: Please refer to Appendix B.

Note that the solution given in Lemma 2 is found under the assumption that $\xi_{v}=1, \forall v$. That is, $\hat{s}_{v}$ given in Eq. (22) should ensure that $\tau_{v}^{\star}>0, \forall v$, in Eq. (18), i.e.,

$$
\frac{\Lambda s^{b h}\left(\sum_{j=1}^{V} \sqrt[3]{\Gamma_{j}}\right)^{2} \sqrt[3]{\Gamma_{v}}}{\lambda(V \Lambda+\Theta)^{2}}<\frac{\Gamma_{v} s^{b h}}{\Lambda \lambda}
$$

Given the definitions of $\Gamma_{v}, \Lambda$, and $\Theta$, it is interesting to find that the inequality (23) can be finally converted to a constraint on the storage size $Q$ of each SBS, which is formulated as

$$
Q>\max \left\{\frac{N C(\delta, \alpha)\left(\sum_{j=1}^{V} \sqrt[3]{\frac{q_{j}}{q_{v}}}-V\right)}{A(\delta, \alpha)-C(\delta, \alpha)+1}, \forall v\right\} .
$$

The constraint imposed on $Q$ can be expressed in a concise manner in the following theorem.

Theorem 2: To make sure that $\hat{s}_{v}$ in Eq. (22) does become the optimal solution of Problem 4 when $\xi_{v}=1, \forall v$, the sufficient and necessary condition to be satisfied is

$$
Q>Q_{\text {min }} \triangleq \frac{N C(\delta, \alpha)\left(\sum_{j=1}^{V} \sqrt[3]{\frac{q_{j}}{q_{V}}}-V\right)}{A(\delta, \alpha)-C(\delta, \alpha)+1},
$$

where $q_{V}$ is the minimum value in $\mathbf{q}$ according to Eq. (5).

Proof: Please refer to Appendix C.

Remark 2: Observe from Eq. (25) that since $\frac{q_{j}}{q_{V}}$ increases exponentially with $\gamma$ according to Eq. (5), the value of $Q_{\min }$ ensuring $\xi_{v}=1, \forall v$, will increase exponentially with $\gamma / 3$.

Note that we have $Q \leq N$. In the case that $Q_{\min }$ in Eq. (25) is larger than $N$ for a high VR popularity exponent $\gamma$, some VRs with the least popularity will be excluded from the game.

\section{B. Further Discussion on $Q$}

We define a series of variables $U_{v}, \forall v$, as follows:

$$
U_{v} \triangleq \frac{N C(\delta, \alpha)\left(\sum_{j=1}^{v} \sqrt[3]{\frac{q_{j}}{q_{v}}}-v\right)}{A(\delta, \alpha)-C(\delta, \alpha)+1},
$$

and formulate the following lemma. 
Lemma 3: $U_{v}$ is a strictly monotonically-increasing function of $v$, i.e., we have $U_{V}>U_{V-1}>\cdots>U_{1}$.

Proof: Please refer to Appendix D.

For the special case of the previous subsection, the optimal solution for $\xi_{v}=1, \forall v$, is found under the condition that the storage size obeys $Q>U_{V}$. In other words, $Q$ should be large enough such that every VR can participate in the game. However, when $Q$ reduces, some VRs have to leave the game as a result of the increased competition. Then we have the following lemma.

Lemma 4: When $U_{v}<Q \leq U_{v+1}$, the NSP can only retain at most the $v$ VRs of $\mathcal{V}_{1}, \mathcal{V}_{2}, \cdots, \mathcal{V}_{v}$ in the game for achieving its optimal solution.

Proof: Please refer to Appendix E.

From Lemma 4, when we have $U_{v}<Q \leq U_{v+1}$, and given that there are $u$ VRs, $u \leq v$, in the game, we can have an optimal solution for $\mathbf{s}$.

Problem 5: When $U_{v}<Q \leq U_{v+1}$ is satisfied, and given that there are $u, u \leq v$, VRs in the game, we can formulate the following optimization problem as

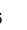

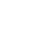

$$
\begin{aligned}
& \min _{\mathbf{s} \succeq \mathbf{0}} \sum_{j=1}^{u} s_{j}, \\
& \text { s.t. } \sum_{j=1}^{u} \sqrt{\frac{\Gamma_{j}}{s_{j}}} \leq(u \Lambda+\Theta) \sqrt{\frac{\lambda}{\Lambda s^{b h}}} .
\end{aligned}
$$

Similar to the solution of Problem 4, we arrive at the optimal solution for the above problem as $\hat{\mathbf{s}}_{u} \triangleq$ $\left[\hat{s}_{1, u}, \cdots, \hat{s}_{i, u}, \cdots, \hat{s}_{V, u}\right]$, where

$$
\hat{s}_{i, u}= \begin{cases}\frac{\Lambda s^{b h}\left(\sum_{j=1}^{u} \sqrt[3]{\Gamma_{j}}\right)^{2} \sqrt[3]{\Gamma_{i}}}{\lambda(u \Lambda+\Theta)^{2}}, & i=1, \cdots, u, \\ \infty, & i=u+1, \cdots, V .\end{cases}
$$

\section{General Case}

Let us now focus our attention on the general solution of the original optimization problem, i.e., of Problem 3. Without loss of generality, we consider the case of $U_{v}<Q \leq U_{v+1}$. Then Problem 3 is equivalent to the following problem.

Problem 6: When $U_{v}<Q \leq U_{v+1}$, there are at most $v$ VRs in the game. Then Problem 3 can be converted to

$$
\begin{aligned}
& \min _{\xi, \mathbf{s} \geq \mathbf{0}} \sum_{j=1}^{v} \xi_{j}\left(\Lambda \lambda s_{j}-\Gamma_{j} s^{b h}\right), \\
& \text { s.t. } \sum_{j=1}^{v} \xi_{j}\left(\sqrt{\frac{\Gamma_{j} \Lambda s^{b h}}{\lambda s_{j}}}-\Lambda\right) \leq \Theta .
\end{aligned}
$$

The problem in Eq. (29) is again non-convex due to the uncertainty of $\xi_{u}, u=1, \cdots, v$. We have to consider the cases, where there are $u, \forall u$, most popular VRs in the game. We observe that for a given $u$, Problem 6 converts to Problem 5. Therefore, to solve Problem 6, we first solve Problem 5 with a given $u$ and obtain $\hat{\mathbf{s}}_{u}$ according to Eq. (28).
TABLE I

The Centralized Algorithm at THE NSP FOR OBTAINING THE OPTIMAL SOLUTION $\mathbf{S}^{\star}$

\section{Algorithm 1 :}

Input: $\quad$ Storage size $Q$, number of videos $N$, VRs' preference distribution q, channel exponent $\alpha$, and pre-set threshold $\delta$.

Output: Optimal pricing vector $\mathbf{s}^{\star}$.

Steps:

1: Based on $N, \mathbf{q}, \alpha$, and $\delta$, the NSP calculates $U_{v}, \forall v$, according to Eq. (26);

2: By comparing $Q$ to $U_{v}$, the NSP obtains the value of the integer $T$ in Eq. (33);

3: Calculate $S_{u}, u=1,2, \cdots, T$, according to Eq. (33);

4: Compare among $S_{1}, \cdots, S_{T}$ for finding the index $\hat{u}$ of the minimum $S_{\hat{u}}$;

5: Based on $\hat{u}, N, \mathbf{q}, \alpha$, and $\delta$, the NSP obtains the optimal solution $\mathbf{s}^{\star}$ according to Eq. (31).

Then we choose the optimal solution, denoted by $\mathbf{s}_{v}^{\star}$, among $\hat{\mathbf{s}}_{1}, \cdots, \hat{\mathbf{s}}_{v}$ as the solution to Problem 6, which is formulated as $\mathbf{s}_{v}^{\star}$

$$
=\arg \min _{\hat{\mathbf{s}}_{u}}\left\{\min \left(\sum_{j=1}^{u}\left(\Lambda \lambda s_{j}-\Gamma_{j} s^{b h}\right)\right), u=1, \cdots, v\right\} .
$$

Based on the above discussions, we can see that the optimal solution $\mathbf{s}^{\star}$ of Problem 3 is a piece-wise function of $Q$, i.e., $\mathbf{s}^{\star}=\mathbf{s}_{v}^{\star}$ when $U_{v}<Q \leq U_{v+1}$. Now, we formulate the solution $\mathbf{s}^{\star}=\left[s_{1}^{\star}, \cdots, s_{V}^{\star}\right]$ to Problem 3 in a general manner as follows.

$$
s_{v}^{\star}=\left\{\begin{array}{cl}
\frac{\Lambda s^{b h}\left(\sum_{j=1}^{\hat{u}} \sqrt[3]{\Gamma_{j}}\right)^{2} \sqrt[3]{\Gamma_{v}}}{\lambda(\hat{u} \Lambda+\Theta)^{2}}, & v=1, \cdots, \hat{u}, \\
\infty, & v=\hat{u}+1, \cdots, V,
\end{array}\right.
$$

where regarding $\hat{u}$, we have

$$
\hat{u}=\arg \min _{u}\left\{S_{u}: u=1,2, \cdots, T\right\},
$$

with $S_{u}$ formulated as

$$
\begin{aligned}
S_{u} & =\sum_{j_{1}=1}^{u}\left(\frac{\Lambda^{2} s^{b h}\left(\sum_{j_{2}}^{u}=1 \sqrt[3]{\Gamma_{j_{2}}}\right)^{2} \sqrt[3]{\Gamma_{j_{1}}}}{(u \Lambda+\Theta)^{2}}-\Gamma_{j_{1}} s^{b h}\right), \\
T & = \begin{cases}1, & U_{1}<Q \leq U_{2} \\
\cdots, & \\
v, & U_{v}<Q \leq U_{v+1} \\
\cdots, & \\
V, & U_{V}<Q\end{cases}
\end{aligned}
$$

To gain a better understanding of the optimal solution in Eq. (31), we propose a centralized algorithm at $\mathcal{L}$ in Table I for obtaining $\mathbf{s}^{\star}$.

Remark 3: The optimal solution $\mathbf{s}^{\star}$ in Eq. (31), combined with the solution of $\boldsymbol{\tau}^{\star}$ given by Eq. (18) in Lemma 1, constitutes the SE for the Stackelberg game. 
Furthermore, by substituting the optimal $\mathbf{s}^{\star}$ into the expression of $S^{N S P}$ in Eq. (19), we get

$$
\begin{aligned}
& S^{N S P}\left(\mathbf{s}^{\star}, \boldsymbol{\tau}^{\star}\right) \\
& =\frac{1}{\Theta} \sum_{j_{1}=1}^{\hat{u}}\left(\Gamma_{j_{1}} s^{b h}-\frac{\Lambda^{2} s^{b h}\left(\sum_{j_{2}=1}^{\hat{u}} \sqrt[3]{\Gamma_{j_{2}}}\right)^{2} \sqrt[3]{\Gamma_{j_{1}}}}{(\hat{u} \Lambda+\Theta)^{2}}\right) .
\end{aligned}
$$

Remark 4: Since we have $\Gamma_{v} \propto q_{v}, \forall v$, and $q_{v}$ increases exponentially with the VR preference parameter $\gamma$ according to Eq. (5), $S^{N S P}\left(\mathbf{s}^{\star}, \boldsymbol{\tau}^{\star}\right)$ also increases exponentially with $\gamma$.

\section{Discussions OF OTHER SCHEMES}

Let us now consider two other schemes, namely, an uniform pricing scheme and a global optimization scheme.

\section{A. Uniform Pricing Scheme}

In contrast to the non-uniform pricing scheme of the previous section, the uniform pricing scheme deliberately imposes the same price on the VRs in the game. We denote the fixed price by $s$. In this case, similar to Lemma 1, Problem 2 can be solved by

$$
\tau_{v}^{\star}=\left(\sqrt{\frac{\Gamma_{v} \Lambda s^{b h}}{\Theta^{2} \lambda}} \sqrt{\frac{1}{s}}-\frac{\Lambda}{\Theta}\right)^{+} .
$$

We first focus our attention on the special case of $\xi_{v}=1, \forall v$. Then Problem 4 can be converted to that of minimizing $s$ subject to the constraint $\sum_{j=1}^{V} \sqrt{\frac{\Gamma_{j}}{s}} \leq(V \Lambda+$ $\Theta) \sqrt{\frac{\lambda}{\Lambda s^{b h}}}$. We then obtain the optimal $\hat{s}$ for this special case as

$$
\hat{s}=\frac{\Lambda s^{b h}\left(\sum_{j=1}^{V} \sqrt{\Gamma_{j}}\right)^{2}}{\lambda(V \Lambda+\Theta)^{2}} .
$$

To guarantee that all the VRs are capable of participating in the game, i.e., $\xi_{v}=1, \forall v$, with the optimal price $\hat{s}$, we let $\hat{s}<\frac{\Gamma_{s} s^{b h}}{\Lambda \lambda}$. Then we have the following constraint on the storage $Q$ as

$$
Q>Q_{\text {min }}^{\prime} \triangleq \frac{N C(\delta, \alpha)\left(\sum_{j=1}^{V} \sqrt{\frac{q_{j}}{q_{V}}}-V\right)}{A(\delta, \alpha)-C(\delta, \alpha)+1} .
$$

We can see that the we require a larger storage size $Q$ in Eq. (37) than that in Eq. (25) under the non-uniform pricing scheme to accommodate all the VRs, since we have $\sum_{j=1}^{V} \sqrt{\frac{q_{j}}{q_{V}}}>\sum_{j=1}^{V} \sqrt[3]{\frac{q_{j}}{q_{V}}}$. Following Remark 2, we conclude that $Q_{\min }^{\prime}$ of the uniform pricing scheme will increase exponentially with $\gamma / 2$.

Then based on this special case, the optimal $\mathbf{s}^{\star}=$ $\left[s_{1}^{\star}, \cdots, s_{V}^{\star}\right]$ in the uniform pricing scheme can be readily obtained by following a similar method to that in the previous section. That is,

$$
s_{v}^{\star}= \begin{cases}\frac{\Lambda s^{b h}\left(\sum_{j=1}^{\hat{u}} \sqrt{\Gamma_{j}}\right)^{2}}{\lambda(\hat{u} \Lambda+\Theta)^{2}}, & v=1, \cdots, \hat{u}, \\ \infty, & v=\hat{u}+1, \cdots, V,\end{cases}
$$

where regarding $\hat{u}$, we have

$$
\hat{u}=\arg \min _{u}\left\{S_{u}: u=1,2, \cdots, T\right\},
$$

with

$$
\begin{aligned}
& S_{u}= \frac{u \Lambda^{2} s^{b h}\left(\sum_{j=1}^{u} \sqrt{\Gamma_{j}}\right)^{2}}{(u \Lambda+\Theta)^{2}}-\sum_{j=1}^{u} \Gamma_{j} s^{b h}, \\
& T= \begin{cases}1, & \bar{U}_{1}<Q \leq \bar{U}_{2}, \\
\cdots, & \bar{U}_{v}<Q \leq \bar{U}_{v+1}, \\
v, & \\
\cdots, & \bar{U}_{V}<Q .\end{cases}
\end{aligned}
$$

Note that $\bar{U}_{v}$ in Eq. (40) is defined as

$$
\bar{U}_{v} \triangleq \frac{N C(\delta, \alpha)\left(\sum_{j=1}^{v} \sqrt{\frac{q_{j}}{q_{v}}}-v\right)}{A(\delta, \alpha)-C(\delta, \alpha)+1} .
$$

It is clear that the uniform pricing scheme is inferior to the non-uniform pricing scheme in terms of maximizing $S^{N S P}$. However, we will show in the following problem that the uniform pricing scheme offers the optimal solution to maximizing the back-haul cost reduction $S^{B H}$ at the NSP in conjunction with $\tau_{v}^{\star}, \forall v$, from the followers.

Problem 7: With the aid of the optimal solutions $\tau_{v}^{\star}, \forall v$, from the followers, the maximization on $S^{B H}$ is achieved by solving the following problem:

$$
\begin{aligned}
& \min _{\xi, \mathbf{s} \geq \mathbf{0}} \sum_{j=1}^{V} \xi_{j}\left(\sqrt{s^{b h}} \sqrt{\Gamma_{j} \Lambda \lambda} \sqrt{s_{j}}-\Gamma_{j} s^{b h}\right), \\
& \text { s.t. } \sum_{j=1}^{V} \xi_{j}\left(\sqrt{\frac{\Gamma_{j} \Lambda s^{b h}}{\lambda s_{j}}}-\Lambda\right) \leq \Theta .
\end{aligned}
$$

The optimal solution to Problem 7 can be readily shown to be $\mathbf{s}^{\star}$ given in Eq. (38). This proof follows the similar procedure of the optimization method presented in the previous section. Thus it is skipped for brevity. In this sense, the uniform pricing scheme is superior to the non-uniform scheme in terms of reducing more cost on back-haul channel transmissions.

\section{B. Global Optimization Scheme}

In the global optimization scheme, we are interested in the sum profit of the NSP and VRs, which can be expressed as

$$
\begin{aligned}
S^{G L B} & =S^{N S P}+\sum_{j=1}^{V} S_{j}^{V R} \\
& =\sum_{j_{1}=1}^{V} \sum_{j_{2}=1}^{F} \frac{2 p_{j_{2}} q_{j_{1}} \zeta K s^{b h} \tau_{j_{1}}}{(A(\delta, \alpha)-C(\delta, \alpha)+1) \tau_{j_{1}}+C(\delta, \alpha) F} \\
& =2 S^{B H} .
\end{aligned}
$$

Observe from Eq. (43), we can see that the sum profit $S^{G L B}$ is twice the back-haul cost reduction $S^{B H}$, where the vector $\tau$ is the only variable of this maximization problem. 667 
Problem 8: The optimization of the sum profit $S^{G L B}$ can be formulated as

$$
\max _{\boldsymbol{\tau} \geq \mathbf{0}} \sum_{j_{1}=1}^{V} \frac{\tau_{j_{1}} \sum_{j_{2}=1}^{F} p_{j_{2}} q_{j_{1}} \zeta K s^{b h}}{(A(\delta, \alpha)-C(\delta, \alpha)+1) \tau_{j_{1}}+C(\delta, \alpha) F},
$$$$
\text { s.t. } \sum_{j=1}^{V} \tau_{j} \leq 1 \text {. }
$$

Problem 8 is a typical water-filling optimization problem. By relying on the classic Lagrangian multiplier, we arrive at the optimal solution as

$$
\hat{\tau}_{v}=\left(\frac{\frac{\sqrt{q_{v}}}{\eta}-C(\delta, \alpha) F}{A(\delta, \alpha)-C(\delta, \alpha)+1}\right)^{+}, \quad \forall v,
$$

where we have $\eta=\frac{\sum_{j=1}^{\bar{v}} \sqrt{q_{j}}}{\bar{v} C(\delta, \alpha) F+A(\delta, \alpha)-C(\delta, \alpha)+1}$, and $\bar{v}$ satisfies the constraint of $\hat{\tau}_{v}>0$.

\section{Comparisons}

Let us now compare the optimal SBS allocation variable $\tau_{v}$ in the context of the above two schemes. First, we investigate $\tau_{v}^{\star}$ in the uniform pricing scheme. By substituting Eq. (38) into Eq. (35), we have

$$
\begin{aligned}
\tau_{v}^{\star} & =\left(\sqrt{\frac{\Gamma_{v} \Lambda s^{b h}}{\Theta^{2} \lambda}} \sqrt{\frac{1}{s_{v}^{\star}}}-\frac{\Lambda}{\Theta}\right)^{+} \\
& = \begin{cases}\frac{\frac{\sqrt{q_{v}}}{\eta^{\prime}}-C(\delta, \alpha) F}{A(\delta, \alpha)-C(\delta, \alpha)+1}, & v=1, \cdots, \hat{u} \\
0, & v=\hat{u}+1, \cdots, V,\end{cases}
\end{aligned}
$$

where $\eta^{\prime}=\frac{\sum_{j=1}^{\hat{u}} \sqrt{q_{j}}}{\hat{u} C(\delta, \alpha) F+A(\delta, \alpha)-C(\delta, \alpha)+1}$, and $\hat{u}$ ensures $\tau_{v}^{\star}>0$.

Then, comparing $\tau_{v}^{\star}$ given in Eq. (46) to the optimal solution $\hat{\tau}$ of the global optimization scheme given by Eq. (45), we can see that these two solutions are the same. In other words, the uniform pricing scheme in fact represents the global optimization scheme in terms of maximizing the sum profit $S^{G L B}$ and maximizing the back-haul cost reduction $S^{B H}$.

\section{NUMERICAL RESULTS}

In this section, we provide both numerical as well as Monte-Carlo simulation results for evaluating the performance of the proposed schemes. The physical layer parameters of our simulations, such as the path-loss exponent $\alpha$, transmit power $P$ of the SBSs and the noise power $\sigma^{2}$ are similar to those of the 3GPP standards. The unit of noise power and transmit power is Watt, while the SBS and MU intensities are expressed in terms of the numbers of the nodes per square kilometer.

Explicitly, we set the path-loss exponent to $\alpha=4$, the SBS transmit power to $P=2$ Watt, the noise power to $\sigma^{2}=10^{-10}$ Watt, and the pre-set SINR threshold to $\delta=0.01$. For the file caching system, we set the number of files in $\mathcal{F}$ to $N=500$ and set the number of VRs to $V=15$. For the network deployments, we set the intensity of the

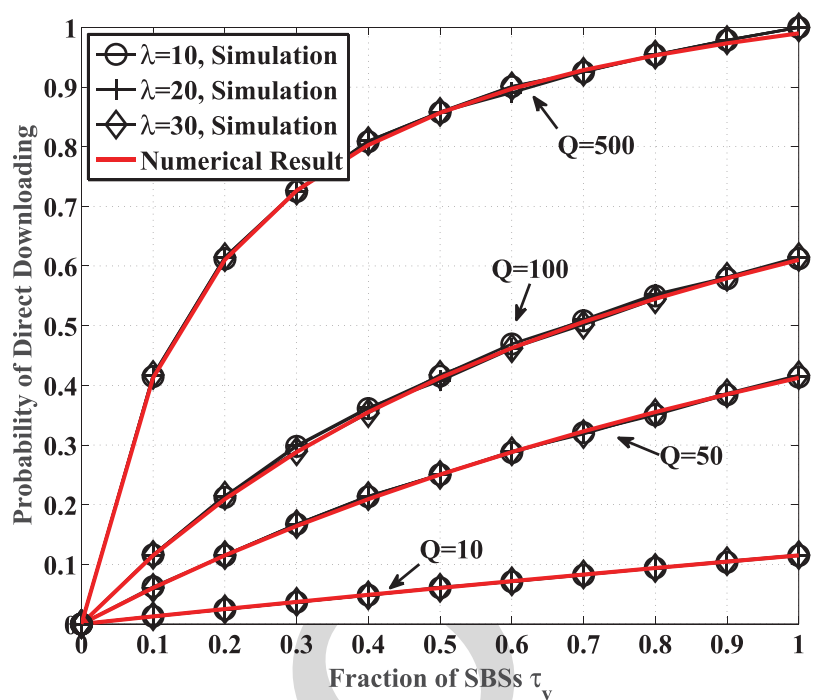

Fig. 2. Comparisons between the simulations and analytical results on $\operatorname{Pr}\left(\mathcal{E}_{v}, f\right)$. We consider four kinds of storage size $Q$ in each SBS, i.e., $Q=10,50,100,500$, and three kinds of SBS intensity, i.e., $\lambda=10,20,30$.

MUs to $\zeta=50 / \mathrm{km}^{2}$, and investigate three cases of the SBS deployments as $\lambda=10 / \mathrm{km}^{2}, 20 / \mathrm{km}^{2}$ and $30 / \mathrm{km}^{2}$.

For the pricing system, the profit/UAP is considered to be the profit gained per month within an area of one square kilometer, i.e., /month $\cdot \mathrm{km}^{2}$. We note that the profits gained by the NSP and by the VRs are proportional to the cost $s^{b h}$ of back-haul channels for transmitting a video. Hence, without loss of generality, we set $s^{b h}=1$ for simplicity. Additionally, we set $K=10 /$ month, which is the average number of video requests from an MU per month.

We first verify our derivation of $\operatorname{Pr}\left(\mathcal{E}_{v, f}\right)$ by comparing the analytical results of Theorem 1 to the Monte-Carlo simulation results. Upon verifying $\operatorname{Pr}\left(\mathcal{E}_{v}, f\right)$, we will investigate the optimization results within the framework of the proposed Stackelberg game by providing numerical results.

\section{A. Performance Evaluation on $\operatorname{Pr}\left(\mathcal{E}_{v, f}\right)$}

For the Monte-Carlo simulations of this subsection, all the average performances are evaluated over a thousand network scenarios, where the distributions of the SBSs and the MUs change from case to case according the PPPs characterized by $\Phi$ and $\Psi$, respectively.

Note that $\operatorname{Pr}\left(\mathcal{E}_{v, f}\right)$ in Theorem 1 is the probability that an MU can obtain its requested video directly from the memory of an SBS rented by $\mathcal{V}_{v}$. We can see from the expression of $\operatorname{Pr}\left(\mathcal{E}_{v}, f\right)$ in Eq. (7) that it is a function of the fraction $\tau_{v}$ of the SBSs that are rented by $\mathcal{V}_{v}$. Although $\tau_{v}$ should be optimized according to the price charged by the NSP, here we investigate a variety of $\tau_{v}$ values, varying from 0 to 1 , to verify the derivation of $\operatorname{Pr}\left(\mathcal{E}_{v, f}\right)$.

Fig. 2 shows our comparisons between the simulations and analytical results on $\operatorname{Pr}\left(\mathcal{E}_{v}, f\right)$. We consider four different storage sizes $Q$ in each SBS by setting $Q=10,50,100,500$. Correspondingly, we have four values for the number of file groups, i.e., $F=50,10,5,1$. Furthermore, we consider the SBS intensities of $\lambda=10,20,30$. From Fig. 2, we can 


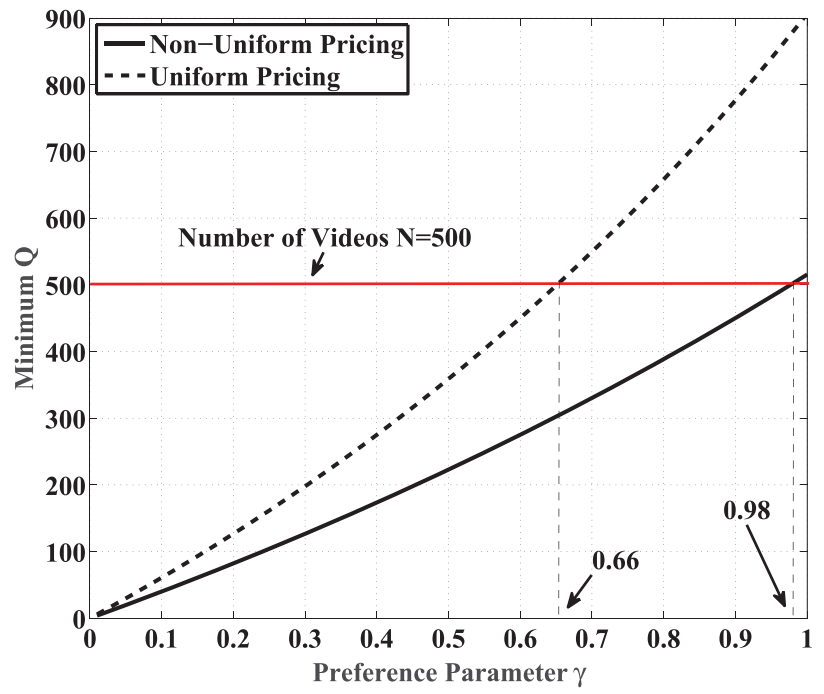

Fig. 3. The minimum number of $Q$ that allows all the VRs to participate in the game under different preference parameter $\gamma$. In the case that the minimum $Q$ is larger than $N$, it means that some VRs will be inevitable excluded from the game.

see that the simulations results closely match the analytical results derived in Theorem 1. Our simulations show that the intensity $\lambda$ does not affect $\operatorname{Pr}\left(\mathcal{E}_{v, f}\right)$, which is consistent with our analytical results. Furthermore, a larger $Q$ leads to a higher value of $\operatorname{Pr}\left(\mathcal{E}_{v}, f\right)$. Hence, enlarging the storage size is helpful for achieving a higher probability of direct downloading.

\section{B. Impact of the VR Preference Parameter $\gamma$}

The preference distribution $\mathbf{q}$ of the VRs defined in Eq. (5) is an important factor in predetermining the system performance. Indeed, we can see from Eq. (5) that this distribution depends on the parameter $\gamma$. Generally, we have $0<\gamma \leq 1$, with a larger $\gamma$ representing a more uneven popularity among the VRs. First, we find the minimum $Q$ that can keep all the VRs in the game. This minimum $Q$ for the non-uniform pricing scheme (NUPS) is given by Eq. (25), while the minimum $Q$ for the uniform-pricing scheme (UPS) is given by Eq. (37). From the two equations, this minimum $Q$ increases exponentially with $\gamma / 3$ in the NUPS, while it also increases exponentially with a higher exponent of $\gamma / 2$ in the UPS. Fig. 3 shows this minimum $Q$ for different values of the VR preference parameter $\gamma$.

We can see that the UPS needs a larger $Q$ than the NUPS for keeping all the VRs. This gap increases rapidly with the growth of $\gamma$. For example, for $\gamma=0.3$, the uniform pricing scheme requires almost 80 more storages, while for $\gamma=0.6$, it needs 200 more. We can also observe in Fig. 3 that for $\gamma>0.66$ in the UPS and for $\gamma>0.98$ in the NUPS, the minimum $Q$ becomes larger than the overall number of videos $N$. In both cases, since we have $Q \leq N$ ( $Q>N$ results in the same performance as $Q=N$ ), some unpopular VRs will be excluded from the game.

Next, we study the number of VR participants that stay in the game for the two schemes upon increasing $\gamma$. We can see from Fig. 4 that the number of VR participants keeps going down upon increasing $\gamma$ in the both schemes. The NUPS

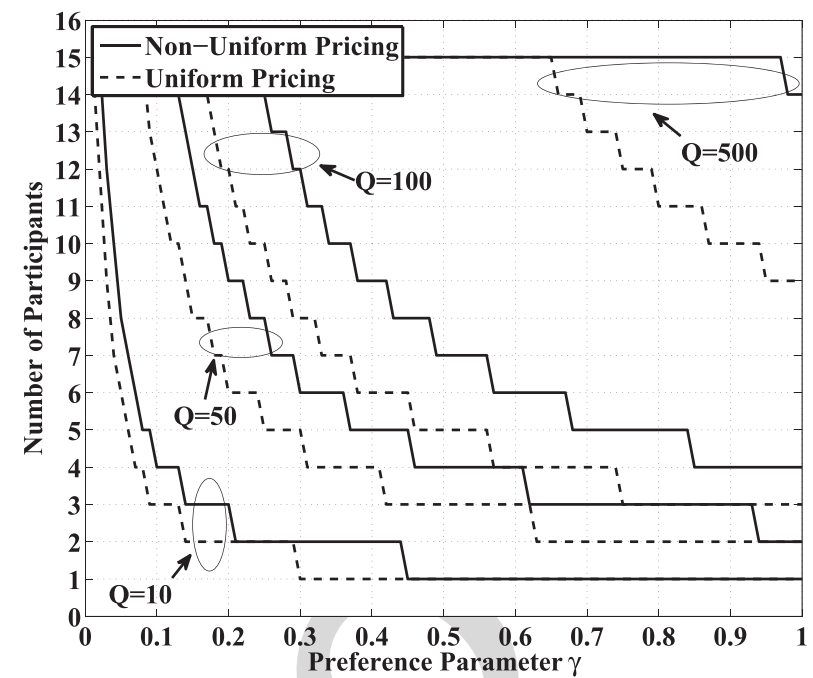

Fig. 4. Number of participants, i.e., the VRs that are in the game, vs. the preference parameter $\gamma$, under the two schemes. We also consider four different values of the storage size $Q$, i.e., $10,50,100,500$.

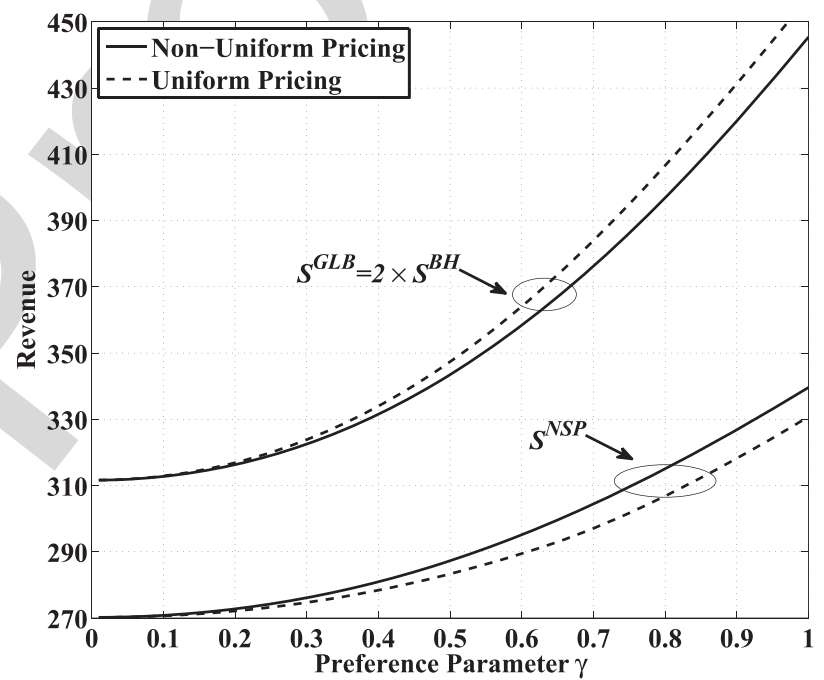

Fig. 5. Various revenues, including $S^{N S P}$ and $S^{G L B}$, vs. the preference parameter $\gamma$, under the two schemes.

always keeps more VRs in the game than the UPS under the same $\gamma$. At the same time, by considering $Q=$ $10,50,100,500$, it is shown that for a given $\gamma$, a higher $Q$ will keep more VRs in the game.

Fig. 5 shows two kinds of revenues gained by the two schemes for a given storage of $Q=500$, namely, the global profit $S^{G L B}$ defined in Eq. (43) and the profit of the NSP $S^{N S P}$ defined in Eq. (9). Recall that we have $S^{G L B}=2 S^{B H}$ according to Eq. (43). We can see that the revenues of both schemes increase exponentially upon increasing $\gamma$, as stated in Remark 4. As our analytical result shows, the profit $S^{N S P}$ gained by the NUPS is optimal and thus it is higher than that gained by the UPS, while the UPS maximizes both $S^{G L B}$ and $S^{B H}$. Fig. 5 verifies the accuracy of our derivations.

\section{Impact of the Storage Size $Q$}

Since $\gamma$ is a network parameter that is relatively fixed, the NSP can adapt the storage size $Q$ for controlling 


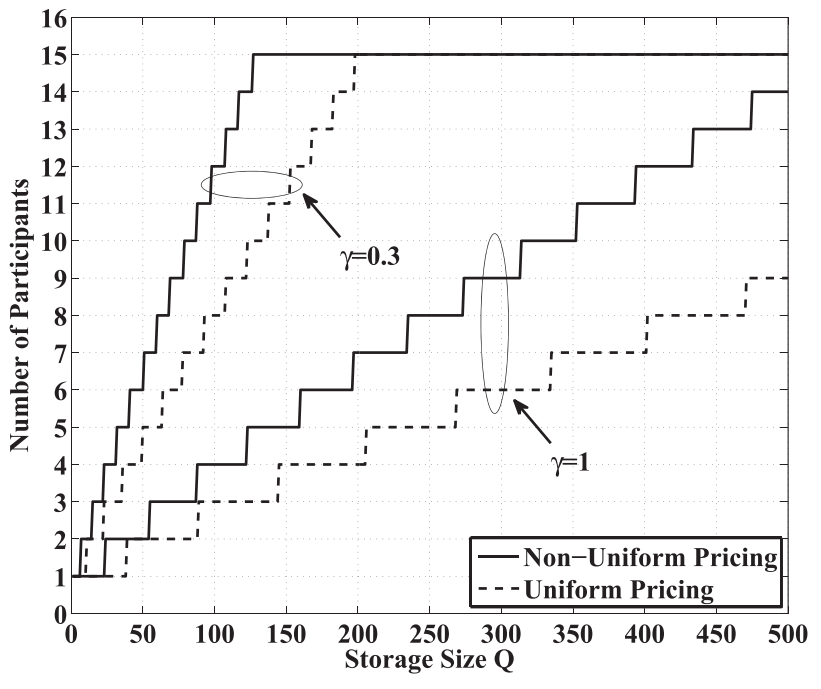

Fig. 6. Number of participants vs. the storage size $Q$, under the two schemes. We also consider two different values of $\gamma$, i.e., $\gamma=0.3,1$.

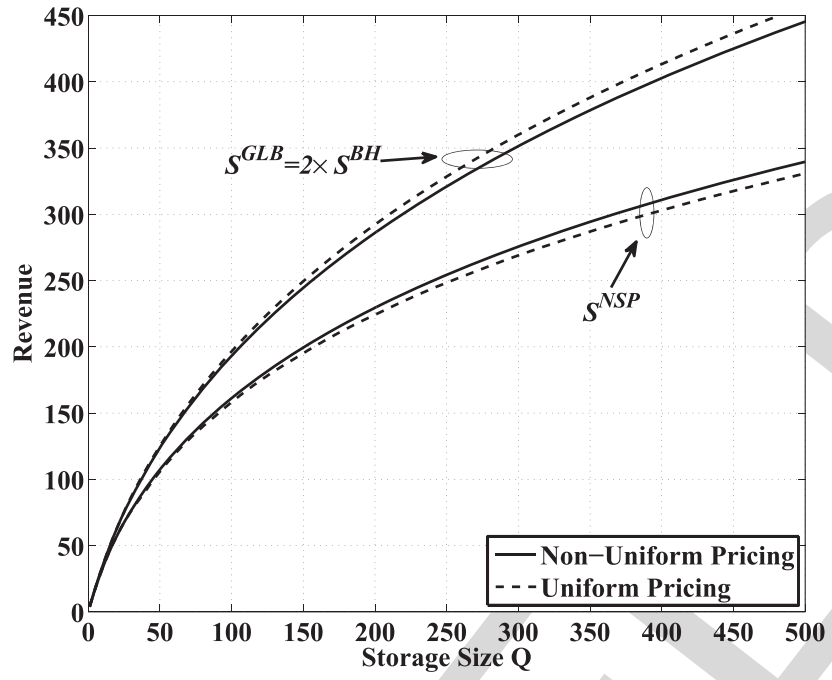

Fig. 7. Various revenues, including $S^{N S P}$ and $S^{G L B}$, vs. the storage size $Q$, under the two schemes. its performance. In this subsection, we investigate the performance as a function of $Q$. Fig. 6 shows the number of participants in the game versus $Q$, where $\gamma=0.3$ and 1 are considered. It is shown that for a larger $Q$, more VRs are able to participate in the game. Again, the NUPS outperforms the UPS owing to its capability of accommodating more VRs for a given $Q$. By comparing the scenarios of $\gamma=0.3$ and 1 , we find that for $\gamma=0.3$, a given increase of $Q$ can accommodate more VRs in the game than $\gamma=1$.

Fig. 7 shows both $S^{N S P}$ and $S^{G L B}$ versus $Q$ for the two schemes for a given $\gamma=1$. We can see that the revenues of both schemes increase with the growth of $Q$. It is shown that the profit $S^{N S P}$ gained by the NUPS is higher than the one gained by the UPS, while the UPS outperforms the NUPS in terms of both $S^{G L B}$ and $S^{B H}$.

\section{Individual VR Performance}

In this subsection, we investigate the performance of each individual VR, including the price charged to them for renting

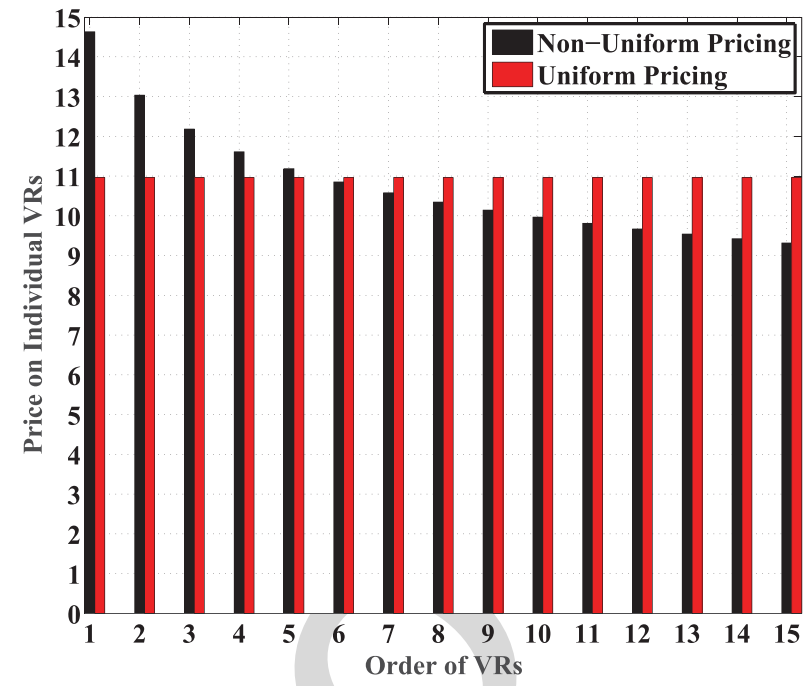

Fig. 8. Price charged on each VR for renting an SBS per month.

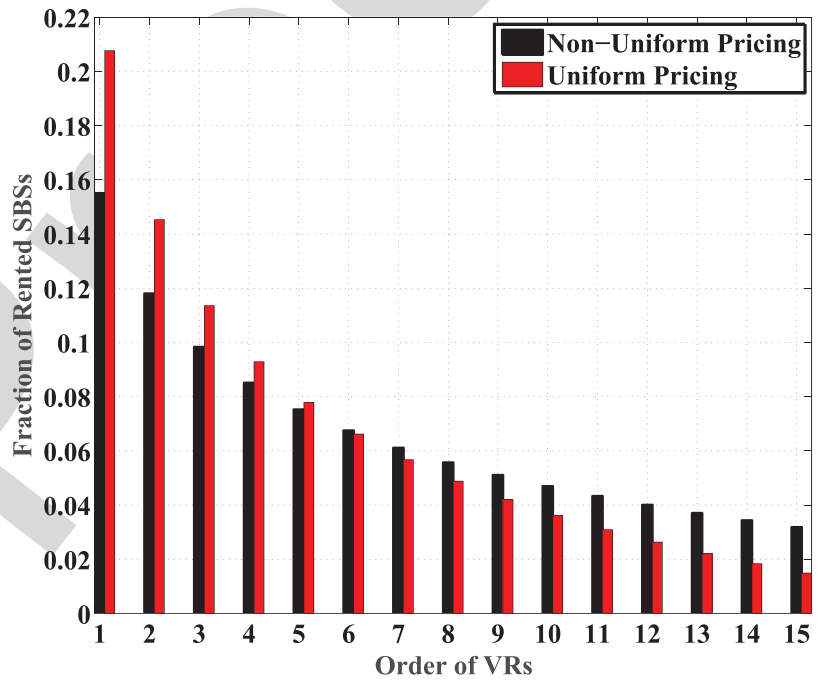

Fig. 9. The fraction of SBSs that are rented by each VR.

an SBS per month, and the fractions of the SBSs they rent from the NSP. We fix $\gamma=0.5$ and choose a large storage size of $Q=500$ for ensuring that all the VRs can be included. Fig. 8 shows the price charged to each VR for renting an SBS. The VRs are arranged according to their popularity order, ranging from $\mathcal{V}_{1}$ to $\mathcal{V}_{15}$, with $\mathcal{V}_{1}$ having the highest popularity and $\mathcal{V}_{15}$ the lowest one. We can see from the figure that in the NUPS, the price for renting an SBS is higher for the VRs having a higher popularity than those with a lower popularity. By contrast, in the UPS, this price is fixed for all the VRs. Fig. 9 shows the specific fraction of the rented SBSs at each VR. In both schemes, the VRs associated with a high popularity tend to rent more SBSs. The UPS in fact represents an instance of the water-filling algorithm. Furthermore, the UPS seems more aggressive than the NUPS, since the less popular VRs of the UPS are more difficult to rent an SBS, and thus these VRs are likely to be excluded from the game with a higher probability.

\section{CONCLUSIONS}

In this paper, we considered a commercial small-cell caching system consisting of an NSP and multiple VRs, where 
the NSP leases its SBSs to the VRs for gaining profits and for reducing the costs of back-haul channel transmissions, while the VRs, after storing popular videos to the rented SBSs, can provide faster transmissions to the MUs, hence gaining more profits. We proposed a Stackelberg game theoretic framework by viewing the SBSs as a type of resources. We first modeled the MUs and SBSs using two independent PPPs with the aid of stochastic geometry, and developed the probability expression of direct downloading. Then, based on the probability derived, we formulated a Stackelberg game for maximizing the average profit of the NSP as well as individual VRs. Next, we investigate the Stackelberg equilibrium by solving the associated nonconvex optimization problem. We considered a non-uniform pricing scheme and an uniform pricing scheme. In the former scheme, the prices charged to each VR for renting an SBS are different, while the latter imposes the same price for each VR. We proved that the non-uniform pricing scheme can effectively maximize the profit of the NSP, while the uniform one maximizes the sum profit of the NSP and the VRs. Furthermore, we derived a relationship between the optimal pricing of renting an SBS, the fraction of SBSs rented by each VR, the storage size of each SBS and the popularity of the VRs. We verified by Monte-Carlo simulations that the direct downloading probability under our PPP model is consistent with our derived results. Then we provided several numerical results for showing that the proposed schemes are effective in both pricing and SBSs allocation.

\section{APPENDIX A}

PROOF OF THEOREM 1

Recall that the SBSs allocated to the VR $\mathcal{V}_{v}$ and cache $\mathcal{G}_{f}$ are modeled as a "thinned" HPPP $\Phi_{v, f}$ having the intensity of $\frac{1}{F} \tau_{v} \lambda$. We consider a typical MU $\mathcal{M}$ who wishes to connect to the nearest SBS $\mathcal{B}$ in $\Phi_{v, f}$. The event $\mathcal{E}_{v, f}$ represents that this SBS can support $\mathcal{M}$ with an SINR no lower than $\delta$, and thus $\mathcal{M}$ can obtain the desired file from the cache of $\mathcal{B}$.

We carry out the analysis on $\operatorname{Pr}\left(\mathcal{E}_{v}, f\right)$ for the typical MU $\mathcal{M}$ located at the origin. Since the network is interference dominant, we neglect the noise in the following. We denote by $z$ the distance between $\mathcal{M}$ and $\mathcal{B}$, by $x_{Z}$ the location of $\mathcal{B}$, and by $\rho\left(x_{Z}\right)$ the received SINR at $\mathcal{M}$ from $\mathcal{B}$. Then the average probability that $\mathcal{M}$ can download the desired video from $\mathcal{B}$ is

$\operatorname{Pr}\left(\rho\left(x_{Z}\right) \geq \delta\right)$

$=\int_{0}^{\infty} \operatorname{Pr}\left(\frac{h_{x_{Z}} z^{-\alpha}}{\sum_{x \in \Phi \backslash\left\{x_{Z}\right\}} h_{x}\|x\|^{-\alpha}} \geq \delta \mid z\right) f_{Z}(z) \mathrm{d} z$

$=\int_{0}^{\infty} \operatorname{Pr}\left(h_{x_{Z}} \geq \frac{\delta\left(\sum_{x \in \Phi \backslash\left\{x_{Z}\right\}} h_{x}\|x\|^{-\alpha}\right)}{z^{-\alpha}} \mid z\right)$

$2 \pi \frac{1}{F} \tau_{v} \lambda z \exp \left(-\pi \frac{1}{F} \tau_{v} \lambda z^{2}\right) \mathrm{d} z$
$=\int_{0}^{\infty} \mathbb{E}_{I}\left(\exp \left(-z^{\alpha} \delta I\right)\right) 2 \pi \frac{1}{F} \tau_{v} \lambda z \exp \left(-\pi \frac{1}{F} \tau_{v} \lambda z^{2}\right) \mathrm{d} z$ where we have $I \triangleq \sum_{x \in \Phi \backslash\left\{x_{Z}\right\}} h_{x}\|x\|^{-\alpha}$, and the PDF of $z$, i.e., $\quad 907$ $f_{Z}(z)$, is derived by the null probability of the HPPP $\Phi_{v, f}$ with the intensity of $\frac{1}{F} \tau_{v} \lambda$. More specifically in $\Phi_{v, f}$, since the number of the SBSs $k$ in an area of $A$ follows the Poisson distribution, the probability of the event that there is no SBS in the area with the radius of $z$ can be calculated as [17]

$$
\operatorname{Pr}\left(k=0 \mid A=\pi z^{2}\right)=e^{-A \frac{1}{F} \tau_{v} \lambda} \frac{\left(A \frac{1}{F} \tau_{v} \lambda\right)^{k}}{k !}=e^{-\pi z^{2} \frac{1}{F} \tau_{v} \lambda} .
$$

By using the above expression, we arrive at $f_{Z}(z)=$ $2 \pi \frac{1}{F} \tau_{v} \lambda z \exp \left(-\pi \frac{1}{F} \tau_{v} \lambda z^{2}\right)$. Note that the interference $I$ consists of $I_{1}$ and $I_{2}$, where $I_{1}$ emanates from the SBSs in $\Phi$ excluding $\Phi_{v, f}$, while $I_{2}$ is from the SBSs in $\Phi_{v, f}$ excluding $\mathcal{B}$. The SBSs contributing to $I_{1}$, denoted by $\Phi \overline{v, f}$, have the intensity of $\left(1-\frac{1}{F} \tau_{v}\right) \lambda$, while those contributing to $I_{2}$ have the intensity of $\frac{1}{F} \tau_{v} \lambda$.

Correspondingly, the calculation of $\mathbb{E}_{I}\left(\exp \left(-z^{\alpha} \delta I\right)\right)$ will be split into the product of two expectations over $I_{1}$ and $I_{2}$. The expectation over $I_{1}$ is calculated as

$$
\begin{aligned}
& \mathbb{E}_{I_{1}}\left(\exp \left(-z^{\alpha} \delta I_{1}\right)\right) \\
& \stackrel{(a)}{=} \mathbb{E}_{\Phi_{\overline{v, f}}}\left(\prod_{x \in \Phi_{\bar{v}}} \int_{0}^{\infty} \exp \left(-z^{\alpha} \delta h_{x}\|x\|^{-\alpha}\right) \exp \left(-h_{x}\right) \mathrm{d} h_{x}\right) \\
& \stackrel{(b)}{=} \exp \left(-\left(1-\frac{1}{F} \tau_{v}\right) \lambda \int_{\mathbb{R}^{2}}\left(1-\frac{1}{1+z^{\alpha} \delta\left\|x_{k}\right\|^{-\alpha}}\right) \mathrm{d} x_{k}\right) \\
& =\exp \left(-2 \pi\left(1-\frac{1}{F} \tau_{v}\right) \lambda \frac{1}{\alpha} z^{2} \delta^{\frac{2}{\alpha}} B\left(\frac{2}{\alpha}, 1-\frac{2}{\alpha}\right)\right) \\
& =\exp \left(-\pi\left(1-\frac{1}{F} \tau_{v}\right) \lambda C(\delta, \alpha) z^{2}\right)
\end{aligned}
$$

where $(a)$ is based on the independence of channel fading, while $(b)$ follows from $\mathbb{E}\left(\prod_{x} u(x)\right)=$ $\exp \left(-\lambda \int_{\mathbb{R}^{2}}(1-u(x)) \mathrm{d} x\right)$, where $x \in \Phi$ and $\Phi$ is an PPP in $\mathbb{R}^{2}$ with the intensity $\lambda[24]$, and $C(\delta, \alpha)$ has been defined as $\frac{2}{\alpha} \delta^{\frac{2}{\alpha}} B\left(\frac{2}{\alpha}, 1-\frac{2}{\alpha}\right)$.

The expectation over $I_{2}$ has to take into account $z$ as the distance from the nearest interfering SBS. Then we have

$$
\begin{aligned}
& \mathbb{E}_{I_{2}}\left(\exp \left(-z^{\alpha} \delta I_{2}\right)\right) \\
& =\exp \left(-\frac{1}{F} \tau_{v} \lambda 2 \pi \int_{z}^{\infty}\left(1-\frac{1}{1+z^{\alpha} \delta r^{-\alpha}}\right) r \mathrm{~d} r\right) \\
& \stackrel{(a)}{=} \exp \left(-\frac{1}{F} \tau_{v} \lambda \pi \delta^{\frac{2}{\alpha}} z^{2} \frac{2}{\alpha} \int_{\delta^{-1}}^{\infty} \frac{\kappa^{\frac{2}{\alpha}-1}}{1+\kappa} \mathrm{d} x\right) \\
& \stackrel{(b)}{=} \exp \left(-\frac{1}{F} \tau_{v} \lambda \pi \delta z^{2} \frac{2}{\alpha-2}{ }_{2} F_{1}\left(1,1-\frac{2}{\alpha} ; 2-\frac{2}{\alpha} ;-\delta\right)\right)
\end{aligned}
$$

where $(a)$ defines $\kappa \triangleq \delta^{-1} z^{-\alpha} r^{\alpha}$, and ${ }_{2} F_{1}(\cdot)$ in $(b)$ is the hypergeometric function. As we defined $A(\delta, \alpha)=\frac{2 \delta}{\alpha-2} \quad 2 F_{1}\left(1,1-\frac{2}{\alpha} ; 2-\frac{2}{\alpha} ;-\delta\right)$, by

.

.

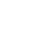

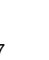


substituting (49) and (50) into (47), we have

$$
\begin{aligned}
& \operatorname{Pr}\left(\rho\left(x_{Z}\right) \geq \delta\right) \\
& =\int_{0}^{\infty} \exp \left(-\pi\left(1-\frac{1}{F} \tau_{v}\right) \lambda C(\delta, \alpha) z^{2}\right) \\
& \quad \exp \left(-\pi \frac{1}{F} \tau_{v} \lambda z^{2} A(\delta, \alpha)\right) 2 \pi \frac{1}{F} \tau_{v} \lambda z \exp \left(-\pi \frac{1}{F} \tau_{v} \lambda z^{2}\right) \mathrm{d} z \\
& =\frac{\frac{1}{F} \tau_{v}}{C(\delta, \alpha)\left(1-\frac{1}{F} \tau_{v}\right)+A(\delta, \alpha) \frac{1}{F} \tau_{v}+\frac{1}{F} \tau_{v}}
\end{aligned}
$$

This completes the proof.

\section{APPENDIX B}

\section{PROOF OF LEMMA 2}

By applying Lagrangian multipliers to the objective function, we have

$L(\mathbf{s}, \mu, \boldsymbol{v})$

$=\sum_{j=1}^{V} s_{j}+\mu\left(\sum_{j=1}^{V} \sqrt{\frac{\Gamma_{j}}{s_{j}}}-(V \Lambda+\Theta) \sqrt{\frac{\lambda}{\Lambda s^{b h}}}\right)-\sum_{j=1}^{V} v_{j} s_{j}$,

where $\mu$ and $v_{j}$ are non-negative multipliers associated with the constraints $\sum_{j=1}^{V} \sqrt{\frac{\Gamma_{j}}{s_{j}}}-(V \Lambda+\Theta) \sqrt{\frac{\lambda}{\Lambda s^{b h}}} \leq 0$ and $s_{j} \geq 0$, respectively. Then the KKT conditions can be written as

$$
\begin{array}{r}
\frac{\partial L(\mathbf{s}, \mu, \boldsymbol{v})}{\partial s_{j}}=0, \quad \forall j=1, \cdots, V, \\
\mu\left(\sum_{j=1}^{V} \sqrt{\frac{\Gamma_{j}}{s_{j}}}-(V \Lambda+\Theta) \sqrt{\frac{\lambda}{\Lambda s^{b h}}}\right)=0, \text { and } v_{j} s_{j}=0, \quad \forall j .
\end{array}
$$

From the first line of Eq. (53), we have

$$
s_{j}=\sqrt[3]{\frac{\mu^{2} \Gamma_{j}}{4\left(1-v_{j}\right)^{2}}} .
$$

Obviously, we have $s_{j} \neq 0, \forall j$, otherwise the constraint $\sum_{j=1}^{V} \sqrt{\frac{\Gamma_{j}}{s_{j}}}-(V \Lambda+\Theta) \sqrt{\frac{\lambda}{\Lambda s^{b h}}} \leq 0$ cannot be satisfied. Thus, we have $v_{j}=0, \forall j$. Furthermore, we have $\mu \neq 0$ according to Eq. (54) since $s_{j}$ is non-zero. This means that $\sum_{j=1}^{V} \sqrt{\frac{\Gamma_{j}}{s_{j}}}-(V \Lambda+\Theta) \sqrt{\frac{\lambda}{\Lambda s^{b h}}}=0$.

By substituting Eq. (54) into this constraint, we have

$$
\sqrt[3]{\mu}=\frac{\sqrt{\Lambda s^{b h}} \sum_{j=1}^{V} \sqrt[3]{2 \Gamma_{j}}}{\sqrt{\lambda}(V \Lambda+\Theta)} .
$$

Then it follows that

$$
s_{j}=\frac{\Lambda s^{b h}\left(\sum_{v=1}^{V} \sqrt[3]{\Gamma_{v}}\right)^{2} \sqrt[3]{\Gamma_{j}}}{\lambda(V \Lambda+\Theta)^{2}} .
$$

This completes the proof.

APPENDIX C Proof of THEOREM 2

As discussed in Eq. (23) and Eq. (24), we have proved that $Q>\frac{N C(\delta, \alpha)\left(\sum_{j=1}^{V} \sqrt[3]{\frac{q_{j}}{q_{V}}}-V\right)}{A(\delta, \alpha)-C(\delta, \alpha)+1}$ is a sufficient condition for the optimal solution in Eq. (22). In other words, as long as $Q$ is satisfied, we have the conclusion that the solution in Eq. (22) is optimal and $\xi_{v}=1, \forall v$.

Next, we prove the necessary aspect. Without loss of ${ }_{983}$ generality, we assume that

$$
\begin{gathered}
\frac{N C(\delta, \alpha)\left(\sum_{j=1}^{V-1} \sqrt[3]{\frac{q_{j}}{q_{V-1}}}-V+1\right)}{A(\delta, \alpha)-C(\delta, \alpha)+1}<Q \\
\leq \frac{N C(\delta, \alpha)\left(\sum_{j=1}^{V} \sqrt[3]{\frac{q_{j}}{q_{V}}}-V\right)}{A(\delta, \alpha)-C(\delta, \alpha)+1} .
\end{gathered}
$$

This leads to $s_{V} \geq \frac{\Gamma_{v} s h}{\Lambda \lambda}$, and the VR $\mathcal{V}_{V}$ will be excluded from the game. In this case, we have $\xi_{j}=1, j=1, \cdots, V-1$, and Problem 4 will be rewritten as follows.

Problem 9: We rewrite Problem 4 as

$$
\begin{aligned}
& \min _{\mathbf{s} \geq \mathbf{0}} \sum_{j=1}^{V-1} s_{j}, \\
& \text { s.t. } \sum_{j=1}^{V-1} \sqrt{\frac{\Gamma_{j}}{s_{j}}} \leq((V-1) \Lambda+\Theta) \sqrt{\frac{\lambda}{\Lambda s^{b h}}} .
\end{aligned}
$$

Similar to the proof of Lemma 2, and combined with the constraint of $Q$ in Eq. (57), the optimal solution of Problem 9 is given by

$$
\hat{s}_{v}= \begin{cases}\frac{\Lambda s^{b h}\left(\sum_{j=1}^{V-1} \sqrt[3]{\Gamma_{j}}\right)^{2} \sqrt[3]{\Gamma_{v}}}{\lambda((V-1) \Lambda+\Theta)^{2}}, & v=1, \cdots, V-1, \\ \infty, & v=V .\end{cases}
$$

We can see that the optimal solution given in Eq. (59) contradicts to the optimal solution of Problem 4 given in Eq. (22). Hence, $Q>\frac{N C(\delta, \alpha)\left(\sum_{j=1}^{V} \sqrt[3]{\frac{q_{j}}{q_{V}}}-V\right)}{A(\delta, \alpha)-C(\delta, \alpha)+1}$ is a necessary condition for finding the optimal solution in Eq. (22). This completes the proof.

\section{APPENDIX D}

PROOF OF LEMMA 3

Consider $v_{1}, v_{2}=1, \cdots, V$ and $v_{1}=v_{2}+1$. Then we prove that $U_{v_{1}}>U_{v_{2}}$. We have

$$
\begin{aligned}
& U_{v_{1}}=\frac{N C(\delta, \alpha)\left(\sum_{j=1}^{v_{1}} \sqrt[3]{\frac{q_{j}}{q_{v_{1}}}}-v_{1}\right)}{A(\delta, \alpha)-C(\delta, \alpha)+1} \\
& =\frac{N C(\delta, \alpha)\left(\sum_{j=1}^{v_{2}} \sqrt[3]{\frac{q_{j}}{q_{v_{1}}}}-v_{2}+\sum_{j=v_{2}+1}^{v_{1}} \sqrt[3]{\frac{q_{j}}{q_{v_{1}}}}-\left(v_{1}-v_{2}\right)\right)}{A(\delta, \alpha)-C(\delta, \alpha)+1} \\
& =\frac{N C(\delta, \alpha)\left(\sum_{j=1}^{v_{2}} \sqrt[3]{\frac{q_{j}}{q_{v_{1}}}}-v_{2}\right)}{A(\delta, \alpha)-C(\delta, \alpha)+1} \\
& \stackrel{\text { (a) }}{>} \frac{N C(\delta, \alpha)\left(\sum_{j=1}^{v_{2}} \sqrt[3]{\frac{q_{j}}{q_{v_{2}}}}-v_{2}\right)}{A(\delta, \alpha)-C(\delta, \alpha)+1}=U_{v_{2}},
\end{aligned}
$$

992 
where $(a)$ comes from the fact that $q_{v_{1}}<q_{v_{2}}$. This completes the proof.

\section{APPENDIX E \\ PROOF OF LEMMA 4}

It is plausible that if $\mathcal{L}$ can only keep at most $v$ VRs, it has to retain the $v$ most popular VRs to maximize its profit. Let us now prove that if $\mathcal{L}$ keeps $(v+w)$ VRs, $w=1, \cdots, V-v$, in the game, it cannot achieve the optimal solution for $U_{v}<Q \leq U_{v+1}$.

Problem 10: In the case that $\mathcal{L}$ keeps $(v+w)$ VRs, we have the optimization problem of

2

$$
\begin{aligned}
& \min _{\mathbf{s} \succeq \mathbf{0}} \sum_{j=1}^{v+w} s_{j}, \\
& \text { s.t. } \sum_{j=1}^{v+w} \sqrt{\frac{\Gamma_{j}}{s_{j}}} \leq((v+w) \Lambda+\Theta) \sqrt{\frac{\lambda}{\Lambda s^{b h}}} .
\end{aligned}
$$

Similar to the proof of Theorem 2, we obtain that $Q>$ $\frac{N C(\delta, \alpha)\left(\sum_{j=1}^{v+w} \sqrt[3]{\frac{q_{j}}{q_{v}+w}}-(v+w)\right)}{A(\delta, \alpha)-C(\delta, \alpha)+1}=U_{v+w}$ is the necessary condition for the $(v+w)$ VRs to participate in the game. This contradicts to the premise $U_{v}<Q \leq U_{v+1}$, since we have $Q>U_{v+1}$ according to Lemma 3. Let us now consider the cases of $w^{\prime}=0,-1, \cdots, 1-v$. To ensure there are $\left(v+w^{\prime}\right)$ VRs in the game, $Q$ has to satisfy the condition that $Q>U_{v+w^{\prime}}$. Since $Q>U_{v} \geq U_{v+w^{\prime}}$, this implies that given $\left(v+w^{\prime}\right)$ VRs in the game, the NSP can achieve an optimal solution. This completes the proof.

\section{REFERENCES}

[1] N. Golrezaei, A. F. Molisch, A. G. Dimakis, and G. Caire, "Femtocaching and device-to-device collaboration: A new architecture for wireless video distribution," IEEE Commun. Mag., vol. 51, no. 4, pp. 142-149, Apr. 2013.

[2] X. Wang, M. Chen, T. Taleb, A. Ksentini, and V. C. M. Leung, "Cache in the air: Exploiting content caching and delivery techniques for $5 \mathrm{G}$ systems," IEEE Commun. Mag., vol. 52, no. 2, pp. 131-139, Feb. 2014.

[3] M. A. Maddah-Ali and U. Niesen, "Decentralized coded caching attains order-optimal memory-rate tradeoff," in Proc. 51st Annu. Allerton Conf. Commun., Control, Comput. (Allerton), Oct. 2013, pp. 421-427.

[4] N. Golrezaei, P. Mansourifard, A. F. Molisch, and A. G. Dimakis, "Basestation assisted device-to-device communications for high-throughput wireless video networks," IEEE Trans. Wireless Commun., vol. 13, no. 7 , pp. 3665-3676, Jul. 2014.

[5] M. Ji, G. Caire, and A. F. Molisch. (May 2013). "Wireless device-todevice caching networks: Basic principles and system performance." [Online]. Available: http://arxiv.org/abs/1305.5216

[6] M. Ji, G. Caire, and A. F. Molisch, "Optimal throughput-outage tradeoff in wireless one-hop caching networks," in Proc. IEEE Int. Symp. Inf. Theory (ISIT), Jul. 2013, pp. 1461-1465.

[7] P. Gupta and P. R. Kumar, "The capacity of wireless networks," IEEE Trans. Inf. Theory, vol. 46, no. 2, pp. 388-404, Mar. 2000.

[8] F. Boccardi, R. W. Heath, A. Lozano, T. L. Marzetta, and P. Popovski, "Five disruptive technology directions for 5G," IEEE Commun. Mag., vol. 52, no. 2, pp. 74-80, Feb. 2014

[9] A. Damnjanovic et al., "A survey on 3GPP heterogeneous networks," IEEE Wireless Commun., vol. 18, no. 3, pp. 10-21, Jun. 2011.

[10] J. Akhtman and L. Hanzo, "Heterogeneous networking: An enabling paradigm for ubiquitous wireless communications," Proc. IEEE, vol. 98, no. 2, pp. 135-138, Feb. 2010.
[11] S. Bayat, R. H. Y. Louie, Z. Han, B. Vucetic, and Y. Li, "Distributed user association and femtocell allocation in heterogeneous wireless networks," IEEE Trans. Commun., vol. 62, no. 8, pp. 3027-3043, Aug. 2014.

[12] M. Mirahmadi, A. Al-Dweik, and A. Shami, "Interference modeling and performance evaluation of heterogeneous cellular networks," IEEE Trans. Commun., vol. 62, no. 6, pp. 2132-2144, Jun. 2014.

[13] A. K. Gupta, H. S. Dhillon, S. Vishwanath, and J. G. Andrews, "Downlink multi-antenna heterogeneous cellular network with load balancing," IEEE Trans. Commun., vol. 62, no. 11, pp. 4052-4067, Nov. 2014.

[14] M. Liebsch, S. Schmid, and J. Awano, "Reducing backhaul costs for mobile content delivery-An analytical study," in Proc. IEEE Int. Conf. Commun. (ICC), Jun. 2012, pp. 2895-2900.

[15] K. Shanmugam, N. Golrezaei, A. G. Dimakis, A. F. Molisch, an G. Caire, "FemtoCaching: Wireless content delivery through distributed caching helpers," IEEE Trans. Inf. Theory, vol. 59, no. 12, pp. 8402-8413, Dec. 2013.

[16] E. Baştuğ, M. Bennis, and M. Debbah, "Cache-enabled small cell networks: Modeling and tradeoffs," in Proc. 11th Int. Symp. Wireles. Commun. Syst. (ISWCS), Aug. 2014, pp. 649-653.

[17] D. Stoyan, W. S. Kendall, and M. Mecke, Stochastic Geometry and Its Applications. 2nd ed. New York, NY, USA: Wiley, 2003.

[18] M. Haenggi, J. G. Andrews, F. Baccelli, O. Dousse, and M. Franceschetti, "Stochastic geometry and random graphs for the analysis and design of wireless networks," IEEE J. Sel. Areas Commun. vol. 27, no. 7, pp. 1029-1046, Sep. 2009.

[19] G. Vazquez-Vilar, C. Mosquera, and S. K. Jayaweera, "Primary user enters the game: Performance of dynamic spectrum leasing in cognitive radio networks," IEEE Trans. Wireless Commun., vol. 9, no. 12, pp. 3625-3629, Dec. 2010.

[20] X. Kang, R. Zhang, and M. Motani, "Price-based resource allocation for spectrum-sharing femtocell networks: A Stackelberg game approach,' IEEE J. Sel. Areas Commun., vol. 30, no. 3, pp. 538-549, Apr. 2012.

[21] D. Niyato and E. Hossain, "Competitive spectrum sharing in cognitive radio networks: A dynamic game approach," IEEE Trans. Wireless Commun., vol. 7, no. 7, pp. 2651-2660, Jul. 2008

[22] D. Niyato, E. Hossain, and Z. Han, "Dynamics of multiple-seller and multiple-buyer spectrum trading in cognitive radio networks: A gametheoretic modeling approach," IEEE Trans. Mobile Comput., vol. 8, no. 8, pp. 1009-1022, Aug. 2009.

[23] D. Fudenberg and J. Tirole, Game Theory. Cambridge, MA, USA: MIT Press, 1993.

[24] D. J. Daley and D. Vere-Jones, An Introduction to the Theory of Point Processes: Elementary Theory and Methods, vol. 1. Springer, 1996.

[25] M. Cha, H. Kwak, P. Rodriguez, Y.-Y. Ahn, and S. Moon, "iTube, You Tube, everybody tubes: Analyzing the world's largest user generated content video system," in Proc. 7th ACM SIGCOMM Conf. Internet Meas., 2007, pp. 1-14.

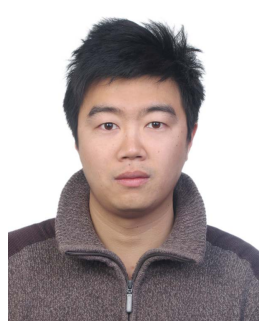

Jun Li (M'09) received the Ph.D. degree in electronics engineering from Shanghai Jiao Tong University, Shanghai, China, in 2009. In 2009, he was with the Department of Research and Innovation, Alcatel Lucent Shanghai Bell, as a Research Scientist. From 2009 to 2012, he was a Post-Doctoral Fellow with the School of Electrical Engineering and Telecommunications, University of New South Wales, Australia. From 2012 to 2015, he was a Research Fellow with the School of Electrical Engineering, The University of Sydney, Australia. Since 2015, he has been a Professor with the School of Electronic and Optical Engineering, Nanjing University of Science and Technology, Nanjing, China. His research interests include network information theory, channel coding theory, wireless network coding, and cooperative communications. 

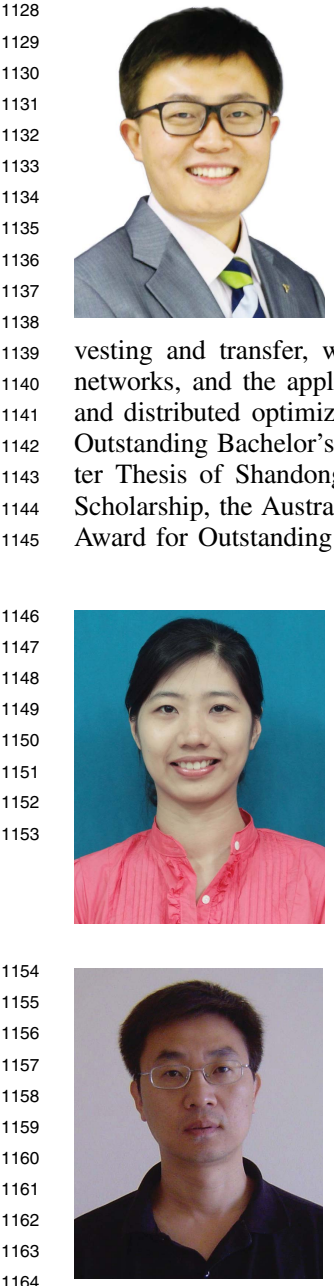

He (Henry) Chen (S'10-M'16) received the B.E. degree in communication engineering and the M.E. degree in communication and information systems from Shandong University, Jinan, China, in 2008 and 2011, respectively, and the Ph.D. degree in electrical engineering from The University of Sydney, Sydney, Australia, in 2015. He is currently a Research Fellow with the School of Electrical and Information Engineering, The University of Sydney. His current research interests include millimeterwave wireless communications, wireless energy harvesting and transfer, wireless network virtualization, cooperative and relay networks, and the applications of game theory, variational inequality theory, and distributed optimization theory in these areas. He was a recipient of the Outstanding Bachelor's Thesis of Shandong University, the Outstanding Master Thesis of Shandong Province, the International Post-Graduate Research Scholarship, the Australian Postgraduate Award, and the Chinese Government Award for Outstanding Self-Financed Students Abroad.

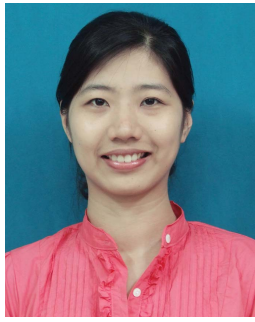

Youjia Chen received the B.S. and M.S. degrees in communication engineering from Nanjing University, Nanjing, China, in 2005 and 2008, respectively. She is currently pursuing the $\mathrm{Ph} . \mathrm{D}$. degree in wireless engineering with The University of Sydney, Sydney, Australia. Her current research interests include resource management, load balancing, and caching strategy in heterogeneous cellular networks.

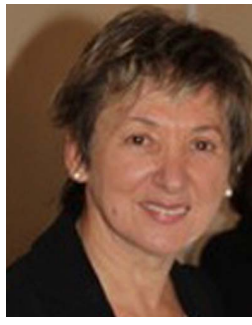

Branka Vucetic (M'83-SM'00-F'03) has held various research and academic positions in Yugoslavia, Australia, U.K., and China. During her career, she co-authored 4 books and more than 400 papers in telecommunications journals and conference proceedings. She currently holds the Peter Nicol Russel Chair of Telecommunications Engineering with The University of Sydney. Her research interests include wireless communications, coding, digital communication theory, and machine-to-machine communications.
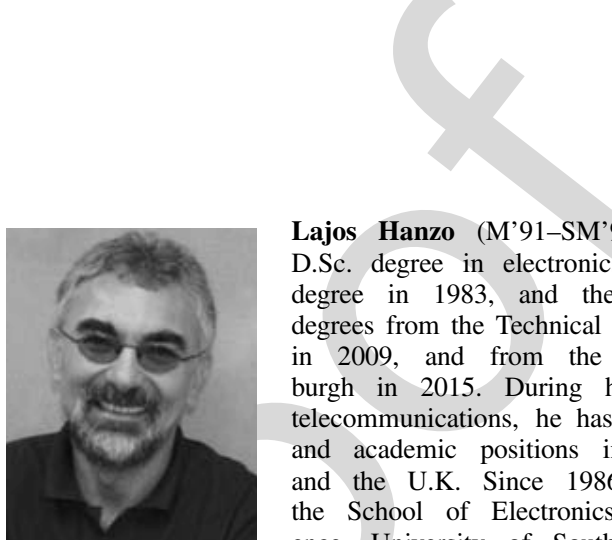

Lajos Hanzo (M'91-SM'92-F'04) received the D.Sc. degree in electronics in 1976, the Ph.D. degree in 1983, and the Honorary Doctorate degrees from the Technical University of Budapest in 2009, and from the University of Edinburgh in 2015. During his 38-year career in telecommunications, he has held various research and academic positions in Hungary, Germany, and the U.K. Since 1986, he has been with the School of Electronics and Computer Science, University of Southampton, U.K., where he holds the Chair in Telecommunications. He has successfully supervised about 100 Ph.D. students, co-authored 20 John Wiley/IEEE Press books on mobile radio communications totaling in excess of 10000 pages, published over 1500 research entries at the IEEE Xplore, acted both as a TPC and General Chair of the IEEE conferences, presented keynote lectures, and has received a number of distinctions. He is currently directing 60 strong academic research teams, working on a range of research projects in the field of wireless multimedia communications sponsored by industry, the Engineering and Physical Sciences Research Council, U.K., the European Research Council's Advanced Fellow Grant, an the Royal Society's Wolfson Research Merit Award. He has 24000 citations. $\mathrm{He}$ is an enthusiastic supporter of industrial and academic liaison. He offers a range of industrial courses. He is also a Governor of the IEEE VTS. From 2008 to 2012, he was the Editor-in-Chief of the IEEE Press and a Chaired Professor with Tsinghua University, Beijing. He is a fellow of REng, IET, and EURASIP.
1179 AQ:6 


\section{AUTHOR QUERIES}

\section{AUTHOR PLEASE ANSWER ALL QUERIES}

PLEASE NOTE: We cannot accept new source files as corrections for your paper. If possible, please annotate the PDF proof we have sent you with your corrections and upload it via the Author Gateway. Alternatively, you may send us your corrections in list format. You may also upload revised graphics via the Author Gateway.

AQ:1 = Please be advised that per instructions from the Communications Society this proof was formatted in Times Roman font and therefore some of the fonts will appear different from the fonts in your originally submitted manuscript. For instance, the math calligraphy font may appear different due to usage of the usepackage[mathcal]euscript. We are no longer permitted to use Computer Modern fonts.

AQ:2 = Please confirm the postal codes for "The University of Sydney and University of Southampton."

AQ:3 = Note that if you require corrections/changes to tables or figures, you must supply the revised files, as these items are not edited for you.

AQ:4 = Please provide the publisher location for ref. [24].

AQ:5 = Please confirm the article title for ref. [25].

AQ:6 = Please confirm whether the edits made in the sentence "Lajos Hanzo received $\ldots$ of Edinburgh in 2015." are OK. 


\title{
Pricing and Resource Allocation via Game Theory for a Small-Cell Video Caching System
}

\author{
Jun Li, Member, IEEE, He Chen, Member, IEEE, Youjia Chen, Zihuai Lin, Senior Member, IEEE, \\ Branka Vucetic, Fellow, IEEE, and Lajos Hanzo, Fellow, IEEE
}

\begin{abstract}
Evidence indicates that downloading on-demand videos accounts for a dramatic increase in data traffic over cellular networks. Caching popular videos in the storage of smallcell base stations (SBS), namely, small-cell caching, is an efficient technology for reducing the transmission latency while mitigating the redundant transmissions of popular videos over back-haul channels. In this paper, we consider a commercialized small-cell caching system consisting of a network service provider (NSP), several video retailers (VRs), and mobile users (MUs). The NSP leases its SBSs to the VRs for the purpose of making profits, and the VRs, after storing popular videos in the rented SBSs, can provide faster local video transmissions to the MUs, thereby gaining more profits. We conceive this system within the framework of Stackelberg game by treating the SBSs as specific types of resources. We first model the MUs and SBSs as two independent Poisson point processes, and develop, via stochastic geometry theory, the probability of the specific event that an MU obtains the video of its choice directly from the memory of an SBS. Then, based on the probability derived, we formulate a Stackelberg game to jointly maximize the average profit of both the NSP and the VRs. In addition, we investigate the Stackelberg equilibrium by solving a non-convex optimization problem. With the aid of this game theoretic framework, we shed light on the relationship between four important factors: the optimal pricing of leasing an SBS, the SBSs allocation among the VRs, the storage size of the SBSs, and the popularity distribution of the VRs. Monte Carlo simulations show that our stochastic geometry-based analytical results closely match the empirical ones. Numerical results are also provided for quantifying the proposed game-theoretic framework by showing its efficiency on pricing and resource allocation.
\end{abstract}

Manuscript received May 28, 2015; revised November 30, 2015; accepted February 16, 2016. This work was supported in part by the National Natural Science Foundation of China under Grant 61501238, Grant 61271230, and Grant 61472190, in part by the Jiangsu Provincial Science Foundation under Project BK20150786, in part by the Specially Appointed Professor Program in Jiangsu Province, 2015, in part by the Open Research Fund of National Key Laboratory of Electromagnetic Environment under Grant 201500013 in part by the Open Research Fund of National Mobile Communications Research Laboratory, Southeast University, under Grant 2013D02, in part by the Australian Research Council under Grant DP120100405 and Grant DP150104019, and in part by the Faculty of Engineering and IT Early Career Researcher Scheme 2016, The University of Sydney. (Corresponding author: Jun Li.)

$\mathrm{J}$. Li is with the School of Electronic and Optical Engineering, Nanjing University of Science and Technology, Nanjing 210094, China (e-mail: jun.li@njust.edu.cn).

H. Chen, Y. Chen, Z. Lin, and B. Vucetic are with the School of Electrical and Information Engineering, The University of Sydney, Sydney, NSW 2006, Australia (e-mail: he.chen@sydney.edu.au; youjia.chen@sydney.edu.au; linzihuai@ieee.org; branka.vucetic@sydney.edu.au).

L. Hanzo is with the Department of Electronics and Computer Science, University of Southampton, Southampton SO17 1BJ, U.K. (e-mail: lh@ecs.soton.ac.uk).

Color versions of one or more of the figures in this paper are available online at http://ieeexplore.ieee.org.

Digital Object Identifier 10.1109/JSAC.2016.2577278
Index Terms-Small-cell caching, cellular networks, stochastic geometry, Stackelberg game.

\section{INTRODUCTION}

$\mathbf{W}$ IRELESS data traffic is expected to increase exponentially in the next few years driven by a staggering proliferation of mobile users (MU) and their bandwidthhungry mobile applications. There is evidence that streaming of on-demand videos by the MUs is the major reason for boosting the tele-traffic over cellular networks [1]. According to the prediction of mobile data traffic by Cisco, mobile video streaming will account for $72 \%$ of the overall mobile data traffic by 2019. The on-demand video downloading involves repeated wireless transmission of videos that are requested multiple times by different users in a completely asynchronous manner, which is different from the transmission style of live video streaming.

Often, there are numerous repetitive requests of popular videos from the MUs, such as online blockbusters, leading to redundant video transmissions. The redundancy of data transmissions can be reduced by locally storing popular videos, known as caching, into the storage of intermediate network nodes, effectively forming a local caching system [1], [2]. The local caching brings video content closer to the MUs and alleviates redundant data transmissions via redirecting the downloading requests to the intermediate nodes.

Generally, wireless data caching consists of two stages: data placement and data delivery [3]. In the data placement stage, popular videos are cached into local storages during off-peak periods, while during the data delivery stage, videos requested are delivered from the local caching system to the MUs. Recent works advanced the caching solutions of both device-to-device (D2D) networks and wireless sensor networks [4]-[6]. Specifically, in [4] a caching scheme was proposed for a D2D based cellular network relaying on the MUs' caching of popular video content. In this scheme, the D2D cluster size was optimized for reducing the downloading delay. In [5] and [6], the authors proposed novel caching schemes for wireless sensor networks, where the protocol model of [7] was adopted.

Since small-cell embedded architectures will dominate in future cellular networks, known as heterogeneous networks (HetNet) [8]-[13], caching relying on small-cell base stations (SBS), namely, small-cell caching, constitutes a promising solution for HetNets. The advantages brought about by small-cell caching are threefold. Firstly, popular videos are placed closer to the MUs when they are cached in SBSs, hence 
reducing the transmission latency. Secondly, redundant transmissions over SBSs' back-haul channels, which are usually expensive [14], can be mitigated. Thirdly, the majority of video traffic is offloaded from macro-cell base stations to SBSs.

In [15], a small-cell caching scheme, named 'Femtocaching', is proposed for a cellular network having embedded SBSs, where the data placement at the SBSs is optimized in a centralized manner for the sake of reducing the transmission delay imposed. However, [15] considers an idealized system, where neither the interference nor the impact of wireless channels is taken into account. The associations between the MUs and the SBSs are pre-determined without considering the specific channel conditions encountered. In [16], small-cell caching is investigated in the context of stochastic networks. The average performance is quantified with the aid of stochastic geometry [17], [18], where the distribution of network nodes is modeled by Poisson point process (PPP). However, the caching strategy of [16] assumes that the SBSs cache the same content, hence leading to a sub-optimal solution.

As detailed above, current research on wireless caching mainly considers the data placement issue optimized for reducing the downloading delay. However, the entire caching system design involves numerous issues apart from data placement. From a commercial perspective, it will be more interesting to consider the topics of pricing for video streaming, the rental of local storage, and so on. A commercialized caching system may consist of video retailers (VR), network service providers (NSP) and MUs. The VRs, e.g., Youtube, purchase copyrights from video producers and publish the videos on their web-sites. The NSPs are typically operators of cellular networks, who are in charge of network facilities, such as macro-cell base stations and SBSs.

In such a commercial small-cell caching system, the VRs' revenue is acquired from providing video streaming for the MUs. As the central servers of the VRs, which store the popular videos, are usually located in the backbone networks and far away from the MUs, an efficient solution is to locally cache these videos, thereby gaining more profits from providing faster local transmissions. In turn, these local caching demands raised by the VRs offer the NSPs profitable opportunities from leasing their SBSs. Additionally, the NSPs can save considerable costs due to reduced redundant video transmissions over SBSs' back-haul channels. In this sense, both the VRs and NSPs are the beneficiaries of the local caching system. However, each entity is selfish and wishes to maximize its own benefit, raising a competition and optimization problem among these entities, which can be effectively solved within the framework of game theory.

We note that game theory has been successfully applied to wireless communications for solving resource allocation problems. In [19], the authors propose a dynamic spectrum leasing mechanism via power control games. In [20], a price-based power allocation scheme is proposed for spectrum sharing in Femto-cell networks based on Stackelberg game. Game theoretical power control strategies for maximizing the utility in spectrum sharing networks are studied in [21] and [22].
In this paper, we propose a commercial small-cell caching system consisting of an NSP, multiple VRs and MUs. We optimize such a system within the framework of Stackelberg game by viewing the SBSs as a specific type of resources for the purpose of video caching. Generally speaking, Stackelberg game is a strategic game that consists of a leader and several followers competing with each other for certain resources [23]. The leader moves first and the followers move subsequently. Correspondingly, in our game theoretic caching system, we consider the NSP to be the leader and the VRs as the followers. The NSP sets the price of leasing an SBS, while the VRs compete with each other for renting a fraction of the SBSs.

To the best of the authors' knowledge, our work is the first of its kind that optimizes a caching system with the aid of game theory. Compared to many other game theory based resource allocation schemes, where the power, bandwidth and time slots are treated as the resources, our work has a totally different profit model, established based on our coverage derivations. In particular, our contributions are as follows.

1) By following the stochastic geometry framework of [17] and [18], we model the MUs and SBSs in the network as two different ties of a Poisson point process (PPP) [24]. Under this network model, we define the concept of a successful video downloading event when an MU obtains the requested video directly from the storage of an SBS. Then we quantify the probability of this event based on stochastic geometry theory.

2) Based on the probability derived, we develop a profit model of our caching system and formulate the profits gained by the NSP and the VRs from SBSs leasing and renting.

3) A Stackelberg game is proposed for jointly maximizing the average profit of the NSP and the VRs. Given this game theoretic framework, we investigate a non-uniform pricing scheme, where the price charged to different VRs varies.

4) Then we investigate the Stackelberg equilibrium of this scheme via solving a non-convex optimization problem. It is interesting to observe that the optimal solution is related both to the storage size of each SBS and to the popularity distribution of the VRs.

5) Furthermore, we consider an uniform pricing scheme. We find that although the uniform pricing scheme is inferior to the non-uniform one in terms of maximizing the NSP's profit, it is capable of reducing more backhaul costs compared with the latter and achieves the maximum sum profit of the NSP and the VRs.

The rest of this paper is organized as follows. We describe the system model in Section II and establish the related profit model in Section III. We then formulate Stackelberg game for our small-cell caching system in Section IV. In Section V, we investigate Stackelberg equilibrium for the non-uniform pricing scheme by solving a non-convex optimization problem, while in Section VI, we further consider the uniform pricing scheme. Our simulations and numerical results are detailed in Section VII, while our conclusions are provided in Section VIII. 


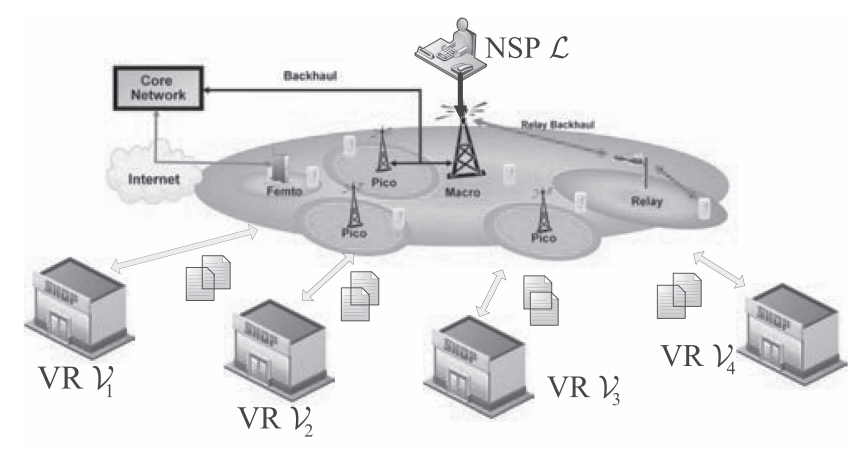

Fig. 1. An example of the small-cell caching system with four VRs.

\section{System Model}

We consider a commercial small-cell caching system consisting of an NSP, $V$ VRs, and a number of MUs. Let us denote by $\mathcal{L}$ the NSP, by $\mathcal{V}=\left\{\mathcal{V}_{1}, \mathcal{V}_{2}, \cdots, \mathcal{V}_{V}\right\}$ the set of the VRs, and by $\mathcal{M}$ one of the MUs. Fig. 1 shows an example of our caching system relying on four VRs. In such a system, the VRs wish to rent the SBSs from $\mathcal{L}$ for placing their videos. Both the NSP and each VR aim for maximizing their profits.

There are three stages in our system. In the first stage, the VRs purchase the copyrights of popular videos from video producers and publish them on their web-sites. In the second stage, the VRs negotiate with the NSP on the rent of SBSs for caching these popular videos. In the third stage, the MUs connect to the SBSs for downloading the desired videos. We will particulary focus our attention on the second and third stages within this game theoretic framework.

\section{A. Network Model}

Let us consider a small-cell based caching network composed of the MUs and the SBSs owned by $\mathcal{L}$, where each SBS is deployed with a fixed transmit power $P$ and the storage of $Q$ video files. Let us assume that the SBSs transmit over the channels that are orthogonal to those of the macro-cell base stations, and thus there is no interference incurred by the macro-cell base stations. Also, assume that these SBSs are spatially distributed according to a homogeneous PPP (HPPP) $\Phi$ of intensity $\lambda$. Here, the intensity $\lambda$ represents the number of the SBSs per unit area. Furthermore, we model the distribution of the MUs as an independent HPPP $\Psi$ of intensity $\zeta$.

The wireless down-link channels spanning from the SBSs to the MUs are independent and identically distributed (i.i.d.), and modeled as the combination of path-loss and Rayleigh fading. Without loss of generality, we carry out our analysis for a typical MU located at the origin. The path-loss between an SBS located at $x$ and the typical MU is denoted by $\|x\|^{-\alpha}$, where $\alpha$ is the path-loss exponent. The channel power of the Rayleigh fading between them is denoted by $h_{x}$, where $h_{x} \sim \exp (1)$. The noise at an MU is Gaussian distributed with a variance $\sigma^{2}$.

We consider the steady-state of a saturated network, where all the SBSs keep on transmitting data in the entire frequency band allocated. This modeling approach for saturated networks characterizes the worst-case scenario of the real systems, which has been adopted by numerous studies on PPP analysis, such as [18]. Hence, the received signal-to-interference-plusnoise ratio (SINR) at the typical MU from an SBS located at $x$ can be expressed as

$$
\rho(x)=\frac{P h_{x}\|x\|^{-\alpha}}{\sum_{x^{\prime} \in \Phi \backslash x} P h_{x^{\prime}}\left\|x^{\prime}\right\|^{-\alpha}+\sigma^{2}} .
$$

The typical MU is considered to be "covered" by an SBS located at $x$ as long as $\rho(x)$ is no lower than a pre-set SINR threshold $\delta$, i.e.,

$$
\rho(x) \geq \delta .
$$

Generally, an MU can be covered by multiple SBSs. Note that the SINR threshold $\delta$ defines the highest delay of downloading a video file. Since the quality and code rate of a video clip have been specified within the video file, the download delay will be the major factor predetermining the QoS perceived by the mobile users. Therefore, we focus our attention on the coverage and SINR in the following derivations.

\section{B. Popularity and Preferences}

We now model the popularity distribution, i.e., the distribution of request probabilities, among the popular videos to be cached. Let us denote by $\mathcal{F}=\left\{\mathcal{F}_{1}, \mathcal{F}_{2}, \cdots, \mathcal{F}_{N}\right\}$ the file set consisting of $N$ video files, where each video file contains an individual movie or video clip that is frequently requested by MUs. The popularity distribution of $\mathcal{F}$ is represented by a vector $\mathbf{t}=\left[t_{1}, t_{2}, \cdots, t_{N}\right]$. That is, the MUs make independent requests of the $n$-th video $\mathcal{F}_{n}, n=1, \cdots, N$, with the probability of $t_{n}$. Generally, $\mathbf{t}$ can be modeled by the Zipf distribution [25] as

$$
t_{n}=\frac{1 / n^{\beta}}{\sum_{j=1}^{N} 1 / j^{\beta}}, \quad \forall n,
$$

where the exponent $\beta$ is a positive value, characterizing the video popularity. A higher $\beta$ corresponds to a higher content reuse, where the most popular files account for the majority of download requests. From Eq. (3), the file with a smaller $n$ corresponds to a higher popularity.

Note that each SBS can cache at most $Q$ video files, and usually $Q$ is no higher than the number of videos in $\mathcal{F}$, i.e., we have $Q \leq N$. Without loss of generality, we assume that $N / Q$ is an integer. The $N$ files in $\mathcal{F}$ are divided into $F=N / Q$ file groups (FG), with each FG containing $Q$ video files. The $n$-th video, $\forall n \in\{(f-1) Q+1, \cdots, f Q\}$, is included in the $f$-th FG, $f=1, \cdots, F$. Denote by $\mathcal{G}_{f}$ the $f$-th FG, and by $p_{f}$ the probability of the MUs' requesting a file in $\mathcal{G}_{f}$, and we have

$$
p_{f}=\sum_{n=(f-1) Q+1}^{f Q} t_{n}, \quad \forall f .
$$

File caching is then carried out on the basis of FGs, where each SBS caches one of the $F$ FGs.

At the same time, the MUs have unbalanced preferences with regard to the $V$ VRs, i.e., some VRs are more popular than others. For example, the majority of the MUs may tend to access Youtube for video streaming. The preference distribution among the VRs is denoted by $\mathbf{q}=\left[q_{1}, q_{2}, \cdots, q_{V}\right]$, 
where $q_{v}, v=1, \cdots, V$, represents the probability that the MUs prefer to download videos from $\mathcal{V}_{v}$. The preference distribution $\mathbf{q}$ can also be modeled by the Zipf distribution. Hence, we have

$$
q_{v}=\frac{1 / v^{\gamma}}{\sum_{j=1}^{V} 1 / j^{\gamma}}, \quad \forall v,
$$

where $\gamma$ is a positive value, characterizing the preference of the VRs. A higher $\gamma$ corresponds to a higher probability of accessing the most popular VRs.

\section{Video Placement and Download}

Next, we introduce the small-cell caching system with its detailed parameters. In the first stage, each VR purchases the $N$ popular videos in $\mathcal{F}$ from the producers and publishes these videos on its web-site. In the second stage, upon obtaining these videos, the VRs negotiate with the NSP $\mathcal{L}$ for renting its SBSs. As $\mathcal{L}$ leases its SBSs to multiple VRs, we denote by $\boldsymbol{\tau}=\left[\tau_{1}, \tau_{2}, \cdots, \tau_{V}\right]$ the fraction vector, where $\tau_{v}$ represents the fraction of the SBSs that are assigned to $\mathcal{V}_{v}, \forall v$. We assume that the SBSs rented by each VR are uniformly distributed. Hence, the SBSs that are allocated to $\mathcal{V}_{v}$ can be modeled as a "thinned" HPPP $\Phi_{v}$ with intensity $\tau_{v} \lambda$.

The data placements of the second stage commence during network off-peak time after the VRs obtain access to the SBSs. During the placements, each SBS will be allocated with one of the $F$ FGs. Generally, we assume that the VRs do not have the a priori information regarding the popularity distribution of $\mathcal{F}$. This is because the popularity of videos is changing periodically, and can only be obtained statistically after these videos quit the market. It is clear that each VR may have more or less some statistical information on the popularity distribution of videos based on the MUs' downloading history. However, this information will be biased due to limited sampling. In this case, the VRs will uniformly assign the $F$ FGs to the SBSs with equal probability of $\frac{1}{F}$ for simplicity. We are interested in investigating the uniform assignment of video files for drawing a bottom line of the system performance. As the FGs are randomly assigned, the SBSs in $\Phi_{v}$ that cache the FG $\mathcal{G}_{f}$ can be further modeled as a "more thinned" HPPP $\Phi_{v, f}$ with an intensity of $\frac{1}{F} \tau_{v} \lambda$.

In the third stage, the MUs start to download videos. When an MU $\mathcal{M}$ requires a video of $\mathcal{G}_{f}$ from $\mathcal{V}_{v}$, it searches the SBSs in $\Phi_{v, f}$ and tries to connect to the nearest SBS that covers $\mathcal{M}$. Provided that such an SBS exists, the MU $\mathcal{M}$ will obtain this video directly from this SBS, and we thereby define this event by $\mathcal{E}_{v, f}$. By contrast, if such an SBS does not exist, $\mathcal{M}$ will be redirected to the central servers of $\mathcal{V}_{v}$ for downloading the requested file. Since the servers of $\mathcal{V}_{v}$ are located at the backbone network, this redirection of the demand will trigger a transmission via the back-haul channels of the NSP $\mathcal{L}$, hence leading to an extra cost.

\section{Profit Modeling}

We now focus on modeling the profit of the NSP and the VRs obtained from the small-cell caching system. The average profit is developed based on stochastically geometrical distributions of the network nodes in terms of per unit area times unit period (/UAP), e.g., /month $\cdot \mathrm{km}^{2}$.

\section{A. Average Profit of the NSP}

For the NSP $\mathcal{L}$, the revenue gained from the caching system consists of two parts: 1) the income gleaned from leasing SBSs to the VRs and 2) the cost reduction due to reduced usage of the SBSs' back-haul channels. First, the leasing income/U AP of $\mathcal{L}$ can be calculated as

$$
S^{R T}=\sum_{j=1}^{V} \tau_{j} \lambda s_{j},
$$

where $s_{j}$ is the price per unit period charged to $\mathcal{V}_{j}$ for renting an SBS. Then we formulate the saved cost/UAP due to reduced back-haul channel transmissions. When an MU demands a video in $\mathcal{G}_{f}$ from $\mathcal{V}_{v}$, we derive the probability $\operatorname{Pr}\left(\mathcal{E}_{v, f}\right)$ as follows.

Theorem 1: The probability of the event $\mathcal{E}_{v, f}, \forall v, f$, can be expressed as

$$
\operatorname{Pr}\left(\mathcal{E}_{v}, f\right)=\frac{\tau_{v}}{C(\delta, \alpha)\left(F-\tau_{v}\right)+A(\delta, \alpha) \tau_{v}+\tau_{v}},
$$

where we have $A(\delta, \alpha) \triangleq \frac{2 \delta}{\alpha-2} \quad{ }_{2} F_{1}\left(1,1-\frac{2}{\alpha} ; 2-\frac{2}{\alpha} ;-\delta\right)$ and $C(\delta, \alpha) \triangleq \frac{2}{\alpha} \delta^{\frac{2}{\alpha}} B\left(\frac{2}{\alpha}, 1-\frac{2}{\alpha}\right)$. Furthermore, ${ }_{2} F_{1}(\cdot)$ in the function $A(\delta, \alpha)$ is the hypergeometric function, while the Beta function in $C(\delta, \alpha)$ is formulated as $B(x, y)=$ $\int_{0}^{1} t^{x-1}(1-t)^{y-1} \mathrm{~d} t$

Proof: Please refer to Appendix A.

Remark 1: From Theorem 1, it is interesting to observe that the probability $\operatorname{Pr}\left(\mathcal{E}_{v}, f\right)$ is independent of both the transmit power $P$ and the intensity $\lambda$ of the SBSs. Furthermore, since $Q$ is inversely proportional to $F$, we can enhance $\operatorname{Pr}\left(\mathcal{E}_{v, f}\right)$ by increasing the storage size $Q$.

We assume that there are on average $K$ video requests from each MU within unit period, and that the average back-haul cost for a video transmission is $s^{b h}$. Based on $\operatorname{Pr}\left(\mathcal{E}_{v, f}\right)$ in Eq. (7), we obtain the cost reduction/UAP for the back-haul channels of $\mathcal{L}$ as

$$
S^{B H}=\sum_{j_{1}=1}^{F} \sum_{j_{2}=1}^{V} p_{j_{1}} q_{j_{2}} \zeta K \operatorname{Pr}\left(\mathcal{E}_{j_{2}, j_{1}}\right) s^{b h} .
$$

By combining the above two items, the overall profit/UAP for $\mathcal{L}$ can be expressed as

$$
S^{N S P}=S^{R T}+S^{B H} .
$$

\section{B. Average Profit of the VRs}

Note that the MUs can download the videos either from the memories of the SBSs directly or from the servers of the VRs at backbone networks via back-haul channels. In the first case, the MUs will be levied by the VRs an extra amount of money in addition to the videos' prices because of the higher-rate local streaming, namely, local downloading surcharge (LDS). We assume that the LDS of each video is set as $s^{l d}$. Then the revenue/UAP for a VR $\mathcal{V}_{v}$ gained from the LDS can be calculated as

$$
S_{v}^{L D}=\sum_{j=1}^{F} p_{j} q_{v} \zeta K \operatorname{Pr}\left(\mathcal{E}_{v, j}\right) s^{l d} .
$$


Additionally, $\mathcal{V}_{v}$ pays for renting the SBSs from $\mathcal{L}$. The related cost/UAP can be written as

$$
S_{v}^{R T}=\tau_{v} \lambda s_{v} .
$$

Upon combining the two items, the profit/UAP for $\mathcal{V}_{v}, \forall v$, can be expressed as

$$
S_{v}^{V R}=S_{v}^{L D}-S_{v}^{R T} .
$$

\section{Problem Formulation}

In this section, we first present the Stackelberg game formulation for our price-based SBS allocation scheme. Then the equilibrium of the proposed game is investigated.

\section{A. Stackelberg Game Formulation}

Again, Stackelberg game is a strategic game that consists of a leader and several followers competing with each other for certain resources [23]. The leader moves first and the followers move subsequently. In our small-cell caching system, we model the NSP $L$ as the leader, and the $V$ VRs as the followers. The NSP imposes a price vector $\mathbf{s}=\left[s_{1}, s_{2}, \cdots, s_{V}\right]$ for the lease of its SBSs, where $s_{v}, \forall v$, has been defined in the previous section as the price per unit period charged on $\mathcal{V}_{v}$ for renting an SBS. After the price vector $\mathbf{s}$ is set, the VRs update the fraction $\tau_{v}, \forall v$, that they tend to rent from $\mathcal{L}$.

1) Optimization Formulation of the Leader: Observe from the above game model that the NSP's objective is to maximize its profit $S^{N S P}$ formulated in Eq. (9). Note that for $\forall v$, the fraction $\tau_{v}$ is a function of the price $s_{v}$ under the Stackelberg game formulation. This means that the fraction of the SBSs that each VR is willing to rent depends on the specific price charged to them for renting an SBS. Consequently, the NSP has to find the optimal price vector $\mathbf{s}$ for maximizing its profit. This optimization problem can be summarized as follows.

Problem 1: The optimization problem of maximizing L's profit can be formulated as

$$
\begin{aligned}
& \max _{\mathbf{s} \geq \mathbf{0}} S^{N S P}(\mathbf{s}, \boldsymbol{\tau}), \\
& \text { s.t. } \sum_{j=1}^{V} \tau_{j} \leq 1 .
\end{aligned}
$$

2) Optimization Formulation of the Followers: The profit gained by the VR $\mathcal{V}_{v}$ in Eq. (12) can be further written as

$$
\begin{aligned}
S_{v}^{V R}\left(\tau_{v}, s_{v}\right)= & \sum_{j=1}^{F} p_{j} q_{v} \zeta K \operatorname{Pr}\left(\mathcal{E}_{v}, j\right) s^{l d}-\tau_{v} \lambda s_{v} \\
= & \sum_{j=1}^{F} \frac{p_{j} q_{v} \zeta K s^{l d} \tau_{v}}{(A(\delta, \alpha)-C(\delta, \alpha)+1) \tau_{v}+C(\delta, \alpha) F} \\
& -\lambda s_{v} \tau_{v} .
\end{aligned}
$$

We can see from Eq. (14) that once the price $s_{v}$ is fixed, the profit of $\mathcal{V}_{v}$ depends on $\tau_{v}$, i.e., the fraction of SBSs that are rented by $\mathcal{V}_{v}$. If $\mathcal{V}_{v}$ increases the fraction $\tau_{v}$, it will gain more revenue by levying surcharges from more MUs, while at the same time, $\mathcal{V}_{v}$ will have to pay for renting more SBSs.
Therefore, $\tau_{v}$ has to be optimized for maximizing the profit of $\mathcal{V}_{v}$. This optimization can be formulated as follows.

Problem 2: The optimization problem of maximizing $\mathcal{V}_{v}$ 's profit can be written as

$$
\max _{\tau_{v} \geq 0} S_{v}^{V R}\left(\tau_{v}, s_{v}\right) .
$$

Problem 1 and Problem 2 together form a Stackelberg game. The objective of this game is to find the Stackelberg Equilibrium (SE) points from which neither the leader (NSP) nor the followers (VRs) have incentives to deviate. In the following, we investigate the SE points for the proposed game.

\section{B. Stackelberg Equilibrium}

For our Stackelberg game, the SE is defined as follows.

Definition 1: Let $\mathbf{s}^{\star} \triangleq\left[s_{1}^{\star}, s_{2}^{\star}, \cdots, s_{V}^{\star}\right]$ be a solution for Problem 1, and $\tau_{v}^{\star}$ be a solution for Problem 2, $\forall v$. Define $\boldsymbol{\tau}^{\star} \triangleq\left[\tau_{1}^{\star}, \tau_{2}^{\star}, \cdots, \tau_{V}^{\star}\right]$. Then the point $\left(\mathbf{s}^{\star}, \boldsymbol{\tau}^{\star}\right)$ is an SE for the proposed Stackelberg game if for any $(\mathbf{s}, \boldsymbol{\tau})$ with $\mathbf{s} \succeq \mathbf{0}$ and $\boldsymbol{\tau} \succeq \mathbf{0}$, the following conditions are satisfied:

$$
\begin{aligned}
S^{N S P}\left(\mathbf{s}^{\star}, \boldsymbol{\tau}^{\star}\right) & \geq S^{N S P}\left(\mathbf{s}, \boldsymbol{\tau}^{\star}\right), \\
S_{v}^{V R}\left(s_{v}^{\star}, \tau_{v}^{\star}\right) & \geq S_{v}^{V R}\left(s_{v}^{\star}, \tau_{v}\right), \quad \forall v .
\end{aligned}
$$

Generally speaking, the SE of a Stackelberg game can be obtained by finding its perfect Nash Equilibrium (NE). In our proposed game, we can see that the VRs strictly compete in a non-cooperative fashion. Therefore, a non-cooperative subgame on controlling the fractions of rented SBSs is formulated at the VRs' side. For a non-cooperative game, the $\mathrm{NE}$ is defined as the operating points at which no players can improve utility by changing its strategy unilaterally. At the NSP's side, since there is only one player, the best response of the NSP is to solve Problem 1. To achieve this, we need to first find the best response functions of the followers, based on which, we solve the best response function for the leader.

Therefore, in our game, we first solve Problem 2 given a price vector $\mathbf{s}$. Then with the obtained best response function $\boldsymbol{\tau}^{\star}$ of the VRs, we solve Problem 1 for the optimal price $\mathbf{s}^{\star}$. In the following, we will have an in-depth investigation on this game theoretic optimization.

\section{GAME Theoretic Optimization}

In this section, we will solve the optimization problem in our game under the non-uniform pricing scheme, where the NSP $\mathcal{L}$ charges the VRs with different prices $s_{1}, \cdots, s_{V}$ for renting an SBS. In this scheme, we first solve Problem 2 at the VRs, and rewrite Eq. (14) as

$$
S_{v}^{V R}\left(\tau_{v}, s_{v}\right)=\frac{\Gamma_{v} s^{l d} \tau_{v}}{\Theta \tau_{v}+\Lambda}-\lambda s_{v} \tau_{v} .0
$$

where $\Gamma_{v} \triangleq \sum_{j=1}^{F} p_{j} q_{v} \zeta K, \Theta \triangleq A(\delta, \alpha)-C(\delta, \alpha)+1$, and $\Lambda \triangleq C(\delta, \alpha) F$. We observe that Eq. (17) is a concave function over the variable $\tau_{v}$. Thus, we can obtain the optimal solution by solving the Karush-Kuhn-Tucker (KKT) conditions, and we have the following lemma. 
Lemma 1: For a given price $s_{v}$, the optimal solution of Problem 2 is

$$
\tau_{v}^{\star}=\left(\sqrt{\frac{\Gamma_{v} \Lambda s^{l d}}{\Theta^{2} \lambda}} \sqrt{\frac{1}{s_{v}}}-\frac{\Lambda}{\Theta}\right)^{+},
$$

where $(\cdot)^{+} \triangleq \max (\cdot, 0)$.

Proof: The optimal solution $\tau_{v}^{\star}$ of $\mathcal{V}_{v}$ can be obtained by deriving $S_{v}^{V R}$ with respect to $\tau_{v}$ and solving $\frac{\mathrm{d} S_{v}^{V R}}{\mathrm{~d} \tau_{v}}=0$ under the constraint that $\tau_{v} \geq 0$.

We can see from Lemma 1 that if the price $s_{v}$ is set too high, i.e., $s_{v} \geq \frac{\Gamma_{v} s^{l d}}{\Lambda \lambda}$, the VR $\mathcal{V}_{v}$ will opt out for renting any SBS from $\mathcal{L}$ due the high price charged. Consequently, the VR $\mathcal{V}_{v}$ will not participate in the game.

In the following derivations, we assume that the LDS on each video $s^{l d}$ is set by the VRs to be the cost of a video transmission via back-haul channels $s^{b h}$. The rational behind this assumption is as follows. Since a local downloading reduce a back-haul transmission, this saved back-haul transmission can be potentially utilized to provide extra services (equivalent to the value of $s^{b h}$ ) for the MUs. In addition, the MUs enjoy the benefit from faster local video transmissions. In light of this, it is reasonable to assume that the MUs are willing to accept the price $s^{b h}$ for a local video transmission.

Substituting the optimal $\tau_{v}^{\star}$ of Eq. (18) into Eq. (9) and carry out some further manipulations, we arrive at

$$
\begin{array}{r}
S^{N S P}=\sum_{j=1}^{V} \lambda s_{j}\left(\sqrt{\frac{\Gamma_{j} \Lambda s^{b h}}{\Theta^{2} \lambda}} \sqrt{\frac{1}{s_{j}}}-\frac{\Lambda}{\Theta}\right)^{+} \\
+\frac{\sum_{i=1}^{F} p_{i} q_{j} \zeta K s^{b h}\left(\sqrt{\frac{\Gamma_{j} \Lambda s^{b h}}{\Theta^{2} \lambda}} \sqrt{\frac{1}{s_{j}}}-\frac{\Lambda}{\Theta}\right)^{+}}{\Theta\left(\sqrt{\frac{\Gamma_{j} \Lambda s^{b h}}{\Theta^{2} \lambda}} \sqrt{\frac{1}{s_{j}}}-\frac{\Lambda}{\Theta}\right)^{+}+\Lambda}
\end{array}
$$

$=\sum_{j=1}^{V} \frac{\xi_{i}}{\Theta}\left(-\Lambda \lambda s_{j}+\left(\sqrt{s^{b h}}-\frac{s^{b h}}{\sqrt{s^{b h}}}\right) \sqrt{\Gamma_{j} \Lambda \lambda s_{j}}+\Gamma_{j} s^{b h}\right)$

$=\sum_{j=1}^{V} \frac{\xi_{i}}{\Theta}\left(-\Lambda \lambda s_{j}+\Gamma_{j} s^{b h}\right)$,

where $\xi_{j}$ is the indicator function, with $\xi_{j}=1$ if $s_{j}<\frac{\Gamma_{j} s^{b h}}{\Lambda \lambda}$ and $\xi_{j}=0$ otherwise. Upon defining the binary vector $\xi \triangleq$ $\left[\xi_{1}, \xi_{2}, \cdots, \xi_{V}\right]$, we can rewrite Problem 1 as follows.

Problem 3: Given the optimal solutions $\tau_{v}^{\star}, \forall v$, gleaned from the followers, we can rewrite Problem 1 as

$$
\begin{aligned}
& \min _{\xi, \mathbf{s} \succeq \mathbf{0}} \sum_{j=1}^{V} \xi_{j}\left(\Lambda \lambda s_{j}-\Gamma_{j} s^{b h}\right), \\
& \text { s.t. } \sum_{j=1}^{V} \xi_{j}\left(\sqrt{\frac{\Gamma_{j} \Lambda s^{b h}}{\lambda s_{j}}}-\Lambda\right) \leq \Theta .
\end{aligned}
$$

Observe from Eq. (20) that Problem 3 is non-convex due to $\xi$. However, for a given $\xi$, this problem can be solved by satisfying the KKT conditions. In the following, we commence with the assumption that $\boldsymbol{\xi}=\mathbf{1}$, i.e., $\xi_{v}=1, \forall v$, and then we extend this result to the general case.

\section{A. Special Case: $\xi_{v}=1, \forall v$}

In this case, all the VRs are participating in the game, and we have the following optimization problem.

Problem 4: Assuming $\xi_{v}=1, \forall v$, we rewrite Problem 3 as

$$
\begin{aligned}
& \min _{\mathbf{s} \geq \mathbf{0}} \sum_{j=1}^{V} s_{j}, \\
& \text { s.t. } \sum_{j=1}^{V} \sqrt{\frac{\Gamma_{j}}{s_{j}}} \leq(V \Lambda+\Theta) \sqrt{\frac{\lambda}{\Lambda s^{b h}}} .
\end{aligned}
$$

The optimal solution of Problem 4 is derived and given in the following lemma.

Lemma 2: The optimal solution to Problem 4 can be derived as $\hat{\mathbf{s}} \triangleq\left[\hat{s}_{1}, \cdots, \hat{s}_{V}\right]$, where

$$
\hat{s}_{v}=\frac{\Lambda s^{b h}\left(\sum_{j=1}^{V} \sqrt[3]{\Gamma_{j}}\right)^{2} \sqrt[3]{\Gamma_{v}}}{\lambda(V \Lambda+\Theta)^{2}}, \quad \forall v .
$$

Proof: Please refer to Appendix B.

Note that the solution given in Lemma 2 is found under the assumption that $\xi_{v}=1, \forall v$. That is, $\hat{s}_{v}$ given in Eq. (22) should ensure that $\tau_{v}^{\star}>0, \forall v$, in Eq. (18), i.e.,

$$
\frac{\Lambda s^{b h}\left(\sum_{j=1}^{V} \sqrt[3]{\Gamma_{j}}\right)^{2} \sqrt[3]{\Gamma_{v}}}{\lambda(V \Lambda+\Theta)^{2}}<\frac{\Gamma_{v} s^{b h}}{\Lambda \lambda} .
$$

Given the definitions of $\Gamma_{v}, \Lambda$, and $\Theta$, it is interesting to find that the inequality (23) can be finally converted to a constraint on the storage size $Q$ of each SBS, which is formulated as

$$
Q>\max \left\{\frac{N C(\delta, \alpha)\left(\sum_{j=1}^{V} \sqrt[3]{\frac{q_{j}}{q_{v}}}-V\right)}{A(\delta, \alpha)-C(\delta, \alpha)+1}, \forall v\right\} .
$$

The constraint imposed on $Q$ can be expressed in a concise manner in the following theorem.

Theorem 2: To make sure that $\hat{s}_{v}$ in Eq. (22) does become the optimal solution of Problem 4 when $\xi_{v}=1, \forall v$, the sufficient and necessary condition to be satisfied is

$$
Q>Q_{\text {min }} \triangleq \frac{N C(\delta, \alpha)\left(\sum_{j=1}^{V} \sqrt[3]{\frac{q_{j}}{q_{V}}}-V\right)}{A(\delta, \alpha)-C(\delta, \alpha)+1},
$$

where $q_{V}$ is the minimum value in $\mathbf{q}$ according to Eq. (5).

Proof: Please refer to Appendix C.

Remark 2: Observe from Eq. (25) that since $\frac{q_{j}}{q_{V}}$ increases exponentially with $\gamma$ according to Eq. (5), the value of $Q_{\min }$ ensuring $\xi_{v}=1, \forall v$, will increase exponentially with $\gamma / 3$.

Note that we have $Q \leq N$. In the case that $Q_{\min }$ in Eq. (25) is larger than $N$ for a high VR popularity exponent $\gamma$, some VRs with the least popularity will be excluded from the game.

\section{B. Further Discussion on $Q$}

We define a series of variables $U_{v}, \forall v$, as follows:

$$
U_{v} \triangleq \frac{N C(\delta, \alpha)\left(\sum_{j=1}^{v} \sqrt[3]{\frac{q_{j}}{q_{v}}}-v\right)}{A(\delta, \alpha)-C(\delta, \alpha)+1},
$$

and formulate the following lemma. 
Lemma 3: $U_{v}$ is a strictly monotonically-increasing function of $v$, i.e., we have $U_{V}>U_{V-1}>\cdots>U_{1}$.

Proof: Please refer to Appendix D.

For the special case of the previous subsection, the optimal solution for $\xi_{v}=1, \forall v$, is found under the condition that the storage size obeys $Q>U_{V}$. In other words, $Q$ should be large enough such that every VR can participate in the game. However, when $Q$ reduces, some VRs have to leave the game as a result of the increased competition. Then we have the following lemma.

Lemma 4: When $U_{v}<Q \leq U_{v+1}$, the NSP can only retain at most the $v$ VRs of $\mathcal{V}_{1}, \mathcal{V}_{2}, \cdots, \mathcal{V}_{v}$ in the game for achieving its optimal solution.

Proof: Please refer to Appendix E.

From Lemma 4, when we have $U_{v}<Q \leq U_{v+1}$, and given that there are $u$ VRs, $u \leq v$, in the game, we can have an optimal solution for $\mathbf{s}$.

Problem 5: When $U_{v}<Q \leq U_{v+1}$ is satisfied, and given that there are $u, u \leq v$, VRs in the game, we can formulate the following optimization problem as

(1)

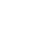

$$
\begin{aligned}
& \min _{\mathbf{s} \succeq \mathbf{0}} \sum_{j=1}^{u} s_{j}, \\
& \text { s.t. } \sum_{j=1}^{u} \sqrt{\frac{\Gamma_{j}}{s_{j}}} \leq(u \Lambda+\Theta) \sqrt{\frac{\lambda}{\Lambda s^{b h}}} .
\end{aligned}
$$

Similar to the solution of Problem 4, we arrive at the optimal solution for the above problem as $\hat{\mathbf{s}}_{u} \triangleq$ $\left[\hat{s}_{1, u}, \cdots, \hat{s}_{i, u}, \cdots, \hat{s}_{V, u}\right]$, where

$$
\hat{s}_{i, u}= \begin{cases}\frac{\Lambda s^{b h}\left(\sum_{j=1}^{u} \sqrt[3]{\Gamma_{j}}\right)^{2} \sqrt[3]{\Gamma_{i}}}{\lambda(u \Lambda+\Theta)^{2}}, & i=1, \cdots, u, \\ \infty, & i=u+1, \cdots, V .\end{cases}
$$

\section{General Case}

Let us now focus our attention on the general solution of the original optimization problem, i.e., of Problem 3. Without loss of generality, we consider the case of $U_{v}<Q \leq U_{v+1}$. Then Problem 3 is equivalent to the following problem.

Problem 6: When $U_{v}<Q \leq U_{v+1}$, there are at most $v$ VRs in the game. Then Problem 3 can be converted to

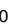

$$
\begin{aligned}
& \min _{\xi, \mathbf{s} \geq \mathbf{0}} \sum_{j=1}^{v} \xi_{j}\left(\Lambda \lambda s_{j}-\Gamma_{j} s^{b h}\right), \\
& \text { s.t. } \sum_{j=1}^{v} \xi_{j}\left(\sqrt{\frac{\Gamma_{j} \Lambda s^{b h}}{\lambda s_{j}}}-\Lambda\right) \leq \Theta .
\end{aligned}
$$

The problem in Eq. (29) is again non-convex due to the uncertainty of $\xi_{u}, u=1, \cdots, v$. We have to consider the cases, where there are $u, \forall u$, most popular VRs in the game. We observe that for a given $u$, Problem 6 converts to Problem 5. Therefore, to solve Problem 6, we first solve Problem 5 with a given $u$ and obtain $\hat{\mathbf{s}}_{u}$ according to Eq. (28).
TABLE I

The Centralized Algorithm AT THE NSP FOR OBTAINING THE OPTIMAL SOLUTION $\mathbf{S}^{\star}$

\section{Algorithm 1 :}

Input: Storage size $Q$, number of videos $N$, VRs' preference distribution q, channel exponent $\alpha$, and pre-set threshold $\delta$.

Output: Optimal pricing vector $\mathbf{s}^{\star}$.

Steps:

1: Based on $N, \mathbf{q}, \alpha$, and $\delta$, the NSP calculates $U_{v}, \forall v$, according to Eq. (26);

2: By comparing $Q$ to $U_{v}$, the NSP obtains the value of the integer $T$ in Eq. (33);

3: Calculate $S_{u}, u=1,2, \cdots, T$, according to Eq. (33);

4: Compare among $S_{1}, \cdots, S_{T}$ for finding the index $\hat{u}$ of the minimum $S_{\hat{u}}$;

5: Based on $\hat{u}, N, \mathbf{q}, \alpha$, and $\delta$, the NSP obtains the optimal solution $\mathbf{s}^{\star}$ according to Eq. (31).

Then we choose the optimal solution, denoted by $\mathbf{s}_{v}^{\star}$, among $\hat{\mathbf{s}}_{1}, \cdots, \hat{\mathbf{s}}_{v}$ as the solution to Problem 6 , which is formulated as $\mathbf{s}_{i}^{\star}$

$$
=\arg \min _{\hat{\mathbf{s}}_{u}}\left\{\min \left(\sum_{j=1}^{u}\left(\Lambda \lambda s_{j}-\Gamma_{j} s^{b h}\right)\right), u=1, \cdots, v\right\} .
$$

Based on the above discussions, we can see that the optimal solution $\mathbf{s}^{\star}$ of Problem 3 is a piece-wise function of $Q$, i.e., $\mathbf{s}^{\star}=\mathbf{s}_{v}^{\star}$ when $U_{v}<Q \leq U_{v+1}$. Now, we formulate the solution $\mathbf{s}^{\star}=\left[s_{1}^{\star}, \cdots, s_{V}^{\star}\right]$ to Problem 3 in a general manner as follows.

$$
s_{v}^{\star}=\left\{\begin{array}{cl}
\frac{\Lambda s^{b h}\left(\sum_{j=1}^{\hat{u}} \sqrt[3]{\Gamma_{j}}\right)^{2} \sqrt[3]{\Gamma_{v}}}{\lambda(\hat{u} \Lambda+\Theta)^{2}}, & v=1, \cdots, \hat{u}, \\
\infty, & v=\hat{u}+1, \cdots, V,
\end{array}\right.
$$

where regarding $\hat{u}$, we have

$$
\hat{u}=\arg \min _{u}\left\{S_{u}: u=1,2, \cdots, T\right\},
$$

with $S_{u}$ formulated as

$$
\begin{aligned}
& S_{u}=\sum_{j_{1}=1}^{u}\left(\frac{\Lambda^{2} s^{b h}\left(\sum_{j_{2}=1}^{u} \sqrt[3]{\Gamma_{j_{2}}}\right)^{2} \sqrt[3]{\Gamma_{j_{1}}}}{(u \Lambda+\Theta)^{2}}-\Gamma_{j_{1}} s^{b h}\right), \\
& T= \begin{cases}1, & U_{1}<Q \leq U_{2}, \\
\cdots, & \\
v, & U_{v}<Q \leq U_{v+1}, \\
\cdots, & \\
V, & U_{V}<Q .\end{cases}
\end{aligned}
$$

To gain a better understanding of the optimal solution in Eq. (31), we propose a centralized algorithm at $\mathcal{L}$ in Table I for obtaining $\mathbf{s}^{\star}$.

Remark 3: The optimal solution $\mathbf{s}^{\star}$ in Eq. (31), combined with the solution of $\boldsymbol{\tau}^{\star}$ given by Eq. (18) in Lemma 1, constitutes the SE for the Stackelberg game. 
Furthermore, by substituting the optimal $\mathbf{s}^{\star}$ into the expression of $S^{N S P}$ in Eq. (19), we get

$$
\begin{aligned}
& S^{N S P}\left(\mathbf{s}^{\star}, \boldsymbol{\tau}^{\star}\right) \\
& =\frac{1}{\Theta} \sum_{j_{1}=1}^{\hat{u}}\left(\Gamma_{j_{1}} s^{b h}-\frac{\Lambda^{2} s^{b h}\left(\sum_{j_{2}=1}^{\hat{u}} \sqrt[3]{\Gamma_{j_{2}}}\right)^{2} \sqrt[3]{\Gamma_{j_{1}}}}{(\hat{u} \Lambda+\Theta)^{2}}\right) .
\end{aligned}
$$

Remark 4: Since we have $\Gamma_{v} \propto q_{v}, \forall v$, and $q_{v}$ increases exponentially with the VR preference parameter $\gamma$ according to Eq. (5), $S^{N S P}\left(\mathbf{s}^{\star}, \boldsymbol{\tau}^{\star}\right)$ also increases exponentially with $\gamma$.

\section{Discussions OF OTHER SCHEMES}

Let us now consider two other schemes, namely, an uniform pricing scheme and a global optimization scheme.

\section{A. Uniform Pricing Scheme}

In contrast to the non-uniform pricing scheme of the previous section, the uniform pricing scheme deliberately imposes the same price on the VRs in the game. We denote the fixed price by $s$. In this case, similar to Lemma 1, Problem 2 can be solved by

$$
\tau_{v}^{\star}=\left(\sqrt{\frac{\Gamma_{v} \Lambda s^{b h}}{\Theta^{2} \lambda}} \sqrt{\frac{1}{s}}-\frac{\Lambda}{\Theta}\right)^{+} .
$$

We first focus our attention on the special case of $\xi_{v}=1, \forall v$. Then Problem 4 can be converted to that of minimizing $s$ subject to the constraint $\sum_{j=1}^{V} \sqrt{\frac{\Gamma_{j}}{s}} \leq(V \Lambda+$ $\Theta) \sqrt{\frac{\lambda}{\Lambda s^{b h}}}$. We then obtain the optimal $\hat{s}$ for this special case as

$$
\hat{s}=\frac{\Lambda s^{b h}\left(\sum_{j=1}^{V} \sqrt{\Gamma_{j}}\right)^{2}}{\lambda(V \Lambda+\Theta)^{2}} .
$$

To guarantee that all the VRs are capable of participating in the game, i.e., $\xi_{v}=1, \forall v$, with the optimal price $\hat{s}$, we let $\hat{s}<\frac{\Gamma_{v} S^{b h}}{\Lambda \lambda}$. Then we have the following constraint on the storage $Q$ as

$$
Q>Q_{\text {min }}^{\prime} \triangleq \frac{N C(\delta, \alpha)\left(\sum_{j=1}^{V} \sqrt{\frac{q_{j}}{q_{V}}}-V\right)}{A(\delta, \alpha)-C(\delta, \alpha)+1} .
$$

We can see that the we require a larger storage size $Q$ in Eq. (37) than that in Eq. (25) under the non-uniform pricing scheme to accommodate all the VRs, since we have $\sum_{j=1}^{V} \sqrt{\frac{q_{j}}{q_{V}}}>\sum_{j=1}^{V} \sqrt[3]{\frac{q_{j}}{q_{V}}}$. Following Remark 2, we conclude that $Q_{\min }^{\prime}$ of the uniform pricing scheme will increase exponentially with $\gamma / 2$.

Then based on this special case, the optimal $\mathbf{s}^{\star}=$ $\left[s_{1}^{\star}, \cdots, s_{V}^{\star}\right]$ in the uniform pricing scheme can be readily obtained by following a similar method to that in the previous section. That is,

$$
s_{v}^{\star}= \begin{cases}\frac{\Lambda s^{b h}\left(\sum_{j=1}^{\hat{u}} \sqrt{\Gamma_{j}}\right)^{2}}{\lambda(\hat{u} \Lambda+\Theta)^{2}}, & v=1, \cdots, \hat{u}, \\ \infty, & v=\hat{u}+1, \cdots, V,\end{cases}
$$

where regarding $\hat{u}$, we have

$$
\hat{u}=\arg \min _{u}\left\{S_{u}: u=1,2, \cdots, T\right\},
$$

with

$$
\begin{aligned}
& S_{u}= \frac{u \Lambda^{2} s^{b h}\left(\sum_{j=1}^{u} \sqrt{\Gamma_{j}}\right)^{2}}{(u \Lambda+\Theta)^{2}}-\sum_{j=1}^{u} \Gamma_{j} s^{b h}, \\
& T= \begin{cases}1, & \bar{U}_{1}<Q \leq \bar{U}_{2}, \\
\cdots, & \bar{U}_{v}<Q \leq \bar{U}_{v+1}, \\
v, & \\
\cdots, & \bar{U}_{V}<Q .\end{cases}
\end{aligned}
$$

Note that $\bar{U}_{v}$ in Eq. (40) is defined as

$$
\bar{U}_{v} \triangleq \frac{N C(\delta, \alpha)\left(\sum_{j=1}^{v} \sqrt{\frac{q_{j}}{q_{v}}}-v\right)}{A(\delta, \alpha)-C(\delta, \alpha)+1} .
$$

It is clear that the uniform pricing scheme is inferior to the non-uniform pricing scheme in terms of maximizing $S^{N S P}$. However, we will show in the following problem that the uniform pricing scheme offers the optimal solution to maximizing the back-haul cost reduction $S^{B H}$ at the NSP in conjunction with $\tau_{v}^{\star}, \forall v$, from the followers.

Problem 7: With the aid of the optimal solutions $\tau_{0}^{\star}, \forall v$, from the followers, the maximization on $S^{B H}$ is achieved by solving the following problem:

$$
\begin{aligned}
& \min _{\boldsymbol{\xi}, \mathbf{s} \geq \mathbf{0}} \sum_{j=1}^{V} \xi_{j}\left(\sqrt{s^{b h}} \sqrt{\Gamma_{j} \Lambda \lambda} \sqrt{s_{j}}-\Gamma_{j} s^{b h}\right), \\
& \text { s.t. } \sum_{j=1}^{V} \xi_{j}\left(\sqrt{\frac{\Gamma_{j} \Lambda s^{b h}}{\lambda s_{j}}}-\Lambda\right) \leq \Theta .
\end{aligned}
$$

The optimal solution to Problem 7 can be readily shown to be $\mathbf{s}^{\star}$ given in Eq. (38). This proof follows the similar procedure of the optimization method presented in the previous section. Thus it is skipped for brevity. In this sense, the uniform pricing scheme is superior to the non-uniform scheme in terms of reducing more cost on back-haul channel transmissions.

\section{B. Global Optimization Scheme}

In the global optimization scheme, we are interested in the sum profit of the NSP and VRs, which can be expressed as

$$
\begin{aligned}
S^{G L B} & =S^{N S P}+\sum_{j=1}^{V} S_{j}^{V R} \\
& =\sum_{j_{1}=1}^{V} \sum_{j_{2}=1}^{F} \frac{2 p_{j_{2}} q_{j_{1}} \zeta K s^{b h} \tau_{j_{1}}}{(A(\delta, \alpha)-C(\delta, \alpha)+1) \tau_{j_{1}}+C(\delta, \alpha) F} \\
& =2 S^{B H} .
\end{aligned}
$$

Observe from Eq. (43), we can see that the sum profit $S^{G L B}$ is twice the back-haul cost reduction $S^{B H}$, where the vector $\tau$ is the only variable of this maximization problem. 67 
Problem 8: The optimization of the sum profit $S^{G L B}$ can be formulated as

$$
\begin{aligned}
& \max _{\boldsymbol{\tau} \geq \mathbf{0}} \sum_{j_{1}=1}^{V} \frac{\tau_{j_{1}} \sum_{j_{2}=1}^{F} p_{j_{2}} q_{j_{1}} \zeta K s^{b h}}{(A(\delta, \alpha)-C(\delta, \alpha)+1) \tau_{j_{1}}+C(\delta, \alpha) F}, \\
& \text { s.t. } \sum_{j=1}^{V} \tau_{j} \leq 1 .
\end{aligned}
$$

Problem 8 is a typical water-filling optimization problem. By relying on the classic Lagrangian multiplier, we arrive at the optimal solution as

$$
\hat{\tau}_{v}=\left(\frac{\frac{\sqrt{q_{v}}}{\eta}-C(\delta, \alpha) F}{A(\delta, \alpha)-C(\delta, \alpha)+1}\right)^{+}, \quad \forall v,
$$

where we have $\eta=\frac{\sum_{j=1}^{\bar{v}} \sqrt{q_{j}}}{\bar{v} C(\delta, \alpha) F+A(\delta, \alpha)-C(\delta, \alpha)+1}$, and $\bar{v}$ satisfies the constraint of $\hat{\tau}_{v}>0$.

\section{Comparisons}

Let us now compare the optimal SBS allocation variable $\tau_{v}$ in the context of the above two schemes. First, we investigate $\tau_{v}^{\star}$ in the uniform pricing scheme. By substituting Eq. (38) into Eq. (35), we have

$$
\begin{aligned}
\tau_{v}^{\star} & =\left(\sqrt{\frac{\Gamma_{v} \Lambda s^{b h}}{\Theta^{2} \lambda}} \sqrt{\frac{1}{s_{v}^{\star}}}-\frac{\Lambda}{\Theta}\right)^{+} \\
& = \begin{cases}\frac{\frac{\sqrt{q_{v}}}{\eta^{\prime}}-C(\delta, \alpha) F}{A(\delta, \alpha)-C(\delta, \alpha)+1}, & v=1, \cdots, \hat{u} \\
0, & v=\hat{u}+1, \cdots, V,\end{cases}
\end{aligned}
$$

where $\eta^{\prime}=\frac{\sum_{j=1}^{\hat{u}} \sqrt{q_{j}}}{\hat{u} C(\delta, \alpha) F+A(\delta, \alpha)-C(\delta, \alpha)+1}$, and $\hat{u}$ ensures $\tau_{v}^{\star}>0$.

Then, comparing $\tau_{v}^{\star}$ given in Eq. (46) to the optimal solution $\hat{\tau}$ of the global optimization scheme given by Eq. (45), we can see that these two solutions are the same. In other words, the uniform pricing scheme in fact represents the global optimization scheme in terms of maximizing the sum profit $S^{G L B}$ and maximizing the back-haul cost reduction $S^{B H}$.

\section{NUMERICAL RESULTS}

In this section, we provide both numerical as well as Monte-Carlo simulation results for evaluating the performance of the proposed schemes. The physical layer parameters of our simulations, such as the path-loss exponent $\alpha$, transmit power $P$ of the SBSs and the noise power $\sigma^{2}$ are similar to those of the 3GPP standards. The unit of noise power and transmit power is Watt, while the SBS and MU intensities are expressed in terms of the numbers of the nodes per square kilometer.

Explicitly, we set the path-loss exponent to $\alpha=4$, the SBS transmit power to $P=2$ Watt, the noise power to $\sigma^{2}=10^{-10}$ Watt, and the pre-set SINR threshold to $\delta=0.01$. For the file caching system, we set the number of files in $\mathcal{F}$ to $N=500$ and set the number of VRs to $V=15$. For the network deployments, we set the intensity of the

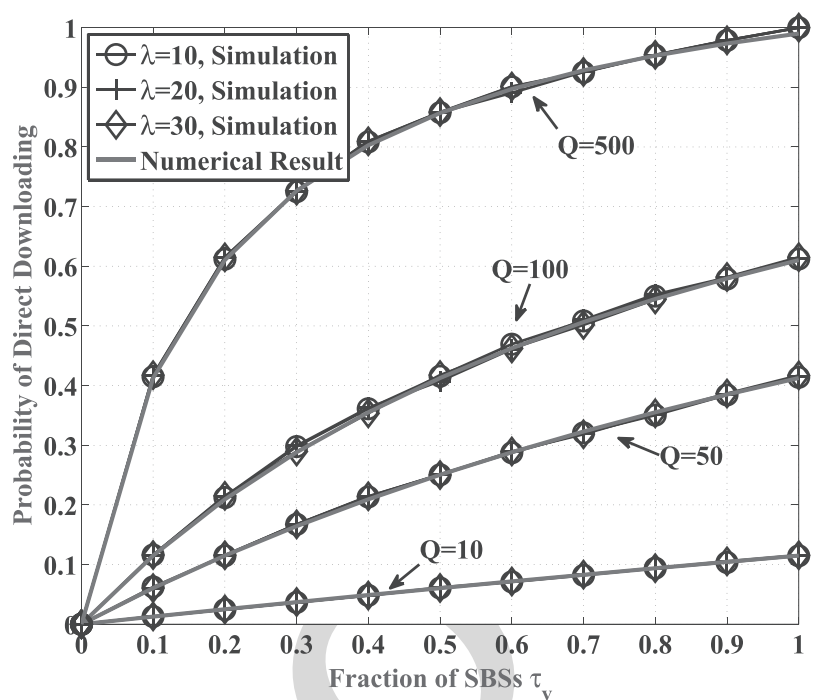

Fig. 2. Comparisons between the simulations and analytical results on $\operatorname{Pr}\left(\mathcal{E}_{v}, f\right)$. We consider four kinds of storage size $Q$ in each SBS, i.e., $Q=10,50,100,500$, and three kinds of SBS intensity, i.e., $\lambda=10,20,30$.

MUs to $\zeta=50 / \mathrm{km}^{2}$, and investigate three cases of the SBS deployments as $\lambda=10 / \mathrm{km}^{2}, 20 / \mathrm{km}^{2}$ and $30 / \mathrm{km}^{2}$.

For the pricing system, the profit/UAP is considered to be the profit gained per month within an area of one square kilometer, i.e., /month $\cdot \mathrm{km}^{2}$. We note that the profits gained by the NSP and by the VRs are proportional to the cost $s^{b h}$ of back-haul channels for transmitting a video. Hence, without loss of generality, we set $s^{b h}=1$ for simplicity. Additionally, we set $K=10 /$ month, which is the average number of video requests from an MU per month.

We first verify our derivation of $\operatorname{Pr}\left(\mathcal{E}_{v, f}\right)$ by comparing the analytical results of Theorem 1 to the Monte-Carlo simulation results. Upon verifying $\operatorname{Pr}\left(\mathcal{E}_{v, f}\right)$, we will investigate the optimization results within the framework of the proposed Stackelberg game by providing numerical results.

\section{A. Performance Evaluation on $\operatorname{Pr}\left(\mathcal{E}_{v, f}\right)$}

For the Monte-Carlo simulations of this subsection, all the average performances are evaluated over a thousand network scenarios, where the distributions of the SBSs and the MUs change from case to case according the PPPs characterized by $\Phi$ and $\Psi$, respectively.

Note that $\operatorname{Pr}\left(\mathcal{E}_{v, f}\right)$ in Theorem 1 is the probability that an MU can obtain its requested video directly from the memory of an SBS rented by $\mathcal{V}_{v}$. We can see from the expression of $\operatorname{Pr}\left(\mathcal{E}_{v}, f\right)$ in Eq. (7) that it is a function of the fraction $\tau_{v}$ of the SBSs that are rented by $\mathcal{V}_{v}$. Although $\tau_{v}$ should be optimized according to the price charged by the NSP, here we investigate a variety of $\tau_{v}$ values, varying from 0 to 1 , to verify the derivation of $\operatorname{Pr}\left(\mathcal{E}_{v, f}\right)$.

Fig. 2 shows our comparisons between the simulations and analytical results on $\operatorname{Pr}\left(\mathcal{E}_{v, f}\right)$. We consider four different storage sizes $Q$ in each SBS by setting $Q=10,50,100,500$. Correspondingly, we have four values for the number of file groups, i.e., $F=50,10,5,1$. Furthermore, we consider the SBS intensities of $\lambda=10,20,30$. From Fig. 2, we can 


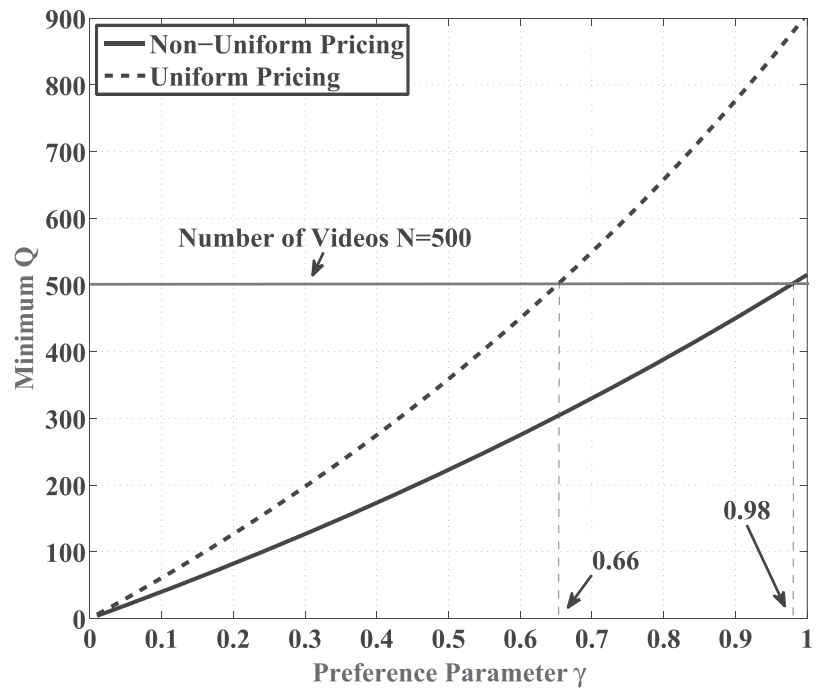

Fig. 3. The minimum number of $Q$ that allows all the VRs to participate in the game under different preference parameter $\gamma$. In the case that the minimum $Q$ is larger than $N$, it means that some VRs will be inevitable excluded from the game.

see that the simulations results closely match the analytical results derived in Theorem 1. Our simulations show that the intensity $\lambda$ does not affect $\operatorname{Pr}\left(\mathcal{E}_{v}, f\right)$, which is consistent with our analytical results. Furthermore, a larger $Q$ leads to a higher value of $\operatorname{Pr}\left(\mathcal{E}_{v}, f\right)$. Hence, enlarging the storage size is helpful for achieving a higher probability of direct downloading.

\section{B. Impact of the VR Preference Parameter $\gamma$}

The preference distribution $\mathbf{q}$ of the VRs defined in Eq. (5) is an important factor in predetermining the system performance. Indeed, we can see from Eq. (5) that this distribution depends on the parameter $\gamma$. Generally, we have $0<\gamma \leq 1$, with a larger $\gamma$ representing a more uneven popularity among the VRs. First, we find the minimum $Q$ that can keep all the VRs in the game. This minimum $Q$ for the non-uniform pricing scheme (NUPS) is given by Eq. (25), while the minimum $Q$ for the uniform-pricing scheme (UPS) is given by Eq. (37). From the two equations, this minimum $Q$ increases exponentially with $\gamma / 3$ in the NUPS, while it also increases exponentially with a higher exponent of $\gamma / 2$ in the UPS. Fig. 3 shows this minimum $Q$ for different values of the VR preference parameter $\gamma$.

We can see that the UPS needs a larger $Q$ than the NUPS for keeping all the VRs. This gap increases rapidly with the growth of $\gamma$. For example, for $\gamma=0.3$, the uniform pricing scheme requires almost 80 more storages, while for $\gamma=0.6$, it needs 200 more. We can also observe in Fig. 3 that for $\gamma>0.66$ in the UPS and for $\gamma>0.98$ in the NUPS, the minimum $Q$ becomes larger than the overall number of videos $N$. In both cases, since we have $Q \leq N$ ( $Q>N$ results in the same performance as $Q=N$ ), some unpopular VRs will be excluded from the game.

Next, we study the number of VR participants that stay in the game for the two schemes upon increasing $\gamma$. We can see from Fig. 4 that the number of VR participants keeps going down upon increasing $\gamma$ in the both schemes. The NUPS

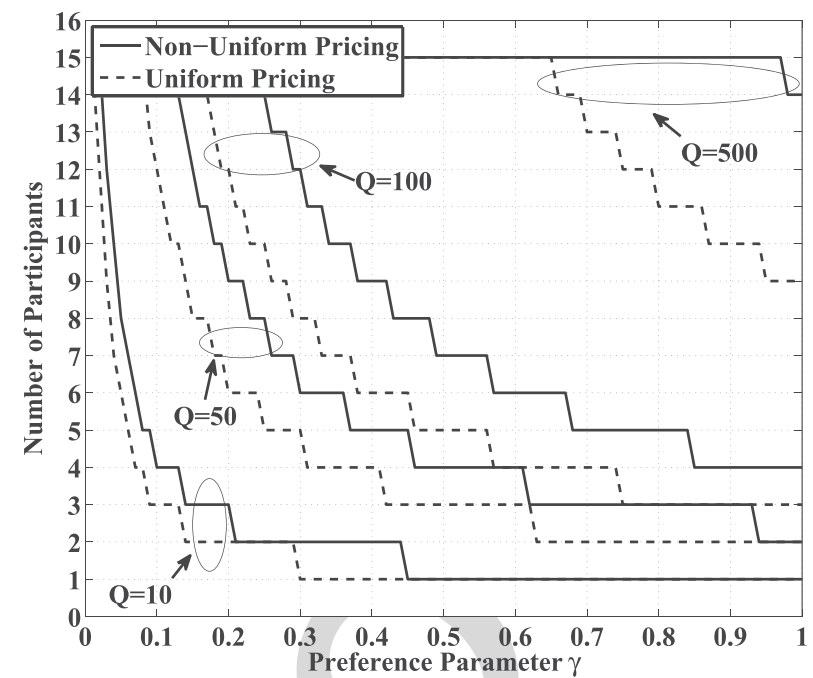

Fig. 4. Number of participants, i.e., the VRs that are in the game, vs. the preference parameter $\gamma$, under the two schemes. We also consider four different values of the storage size $Q$, i.e., $10,50,100,500$.

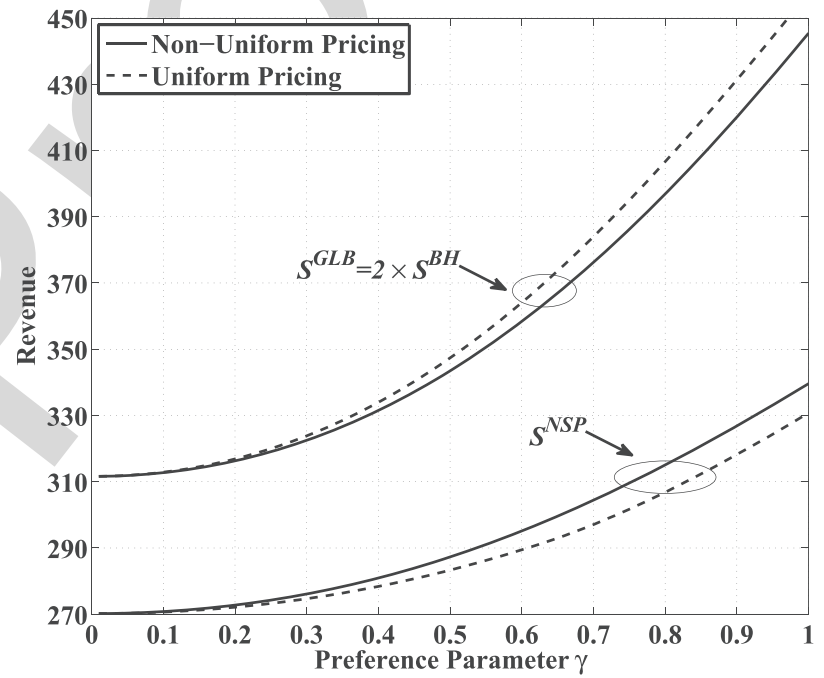

Fig. 5. Various revenues, including $S^{N S P}$ and $S^{G L B}$, vs. the preference parameter $\gamma$, under the two schemes.

always keeps more VRs in the game than the UPS under the same $\gamma$. At the same time, by considering $Q=$ $10,50,100,500$, it is shown that for a given $\gamma$, a higher $Q$ will keep more VRs in the game.

Fig. 5 shows two kinds of revenues gained by the two schemes for a given storage of $Q=500$, namely, the global profit $S^{G L B}$ defined in Eq. (43) and the profit of the NSP $S^{N S P}$ defined in Eq. (9). Recall that we have $S^{G L B}=2 S^{B H}$ according to Eq. (43). We can see that the revenues of both schemes increase exponentially upon increasing $\gamma$, as stated in Remark 4. As our analytical result shows, the profit $S^{N S P}$ gained by the NUPS is optimal and thus it is higher than that gained by the UPS, while the UPS maximizes both $S^{G L B}$ and $S^{B H}$. Fig. 5 verifies the accuracy of our derivations.

\section{Impact of the Storage Size $Q$}

Since $\gamma$ is a network parameter that is relatively fixed, the NSP can adapt the storage size $Q$ for controlling 


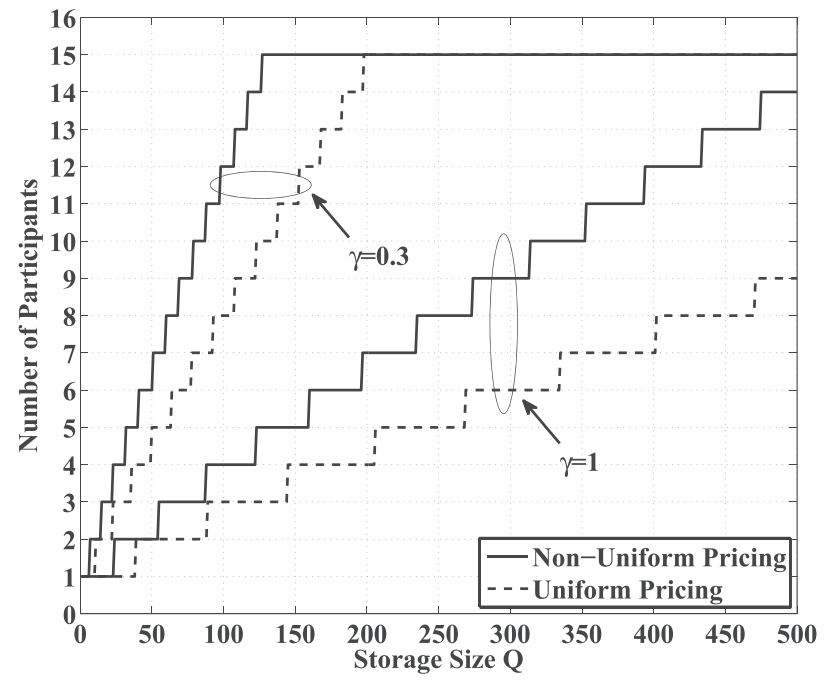

Fig. 6. Number of participants vs. the storage size $Q$, under the two schemes. We also consider two different values of $\gamma$, i.e., $\gamma=0.3,1$.

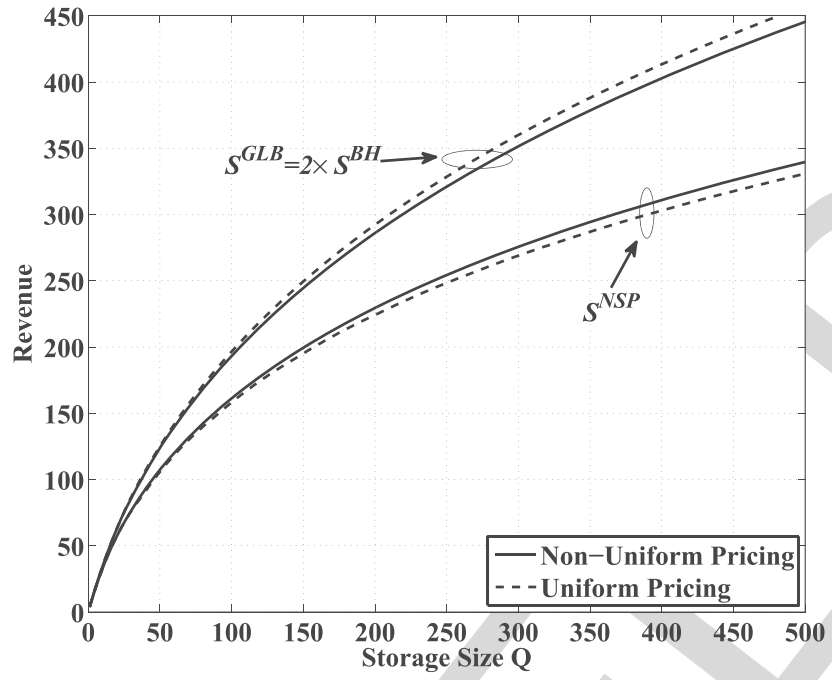

Fig. 7. Various revenues, including $S^{N S P}$ and $S^{G L B}$, vs. the storage size $Q$, under the two schemes. its performance. In this subsection, we investigate the performance as a function of $Q$. Fig. 6 shows the number of participants in the game versus $Q$, where $\gamma=0.3$ and 1 are considered. It is shown that for a larger $Q$, more VRs are able to participate in the game. Again, the NUPS outperforms the UPS owing to its capability of accommodating more VRs for a given $Q$. By comparing the scenarios of $\gamma=0.3$ and 1 , we find that for $\gamma=0.3$, a given increase of $Q$ can accommodate more VRs in the game than $\gamma=1$.

Fig. 7 shows both $S^{N S P}$ and $S^{G L B}$ versus $Q$ for the two schemes for a given $\gamma=1$. We can see that the revenues of both schemes increase with the growth of $Q$. It is shown that the profit $S^{N S P}$ gained by the NUPS is higher than the one gained by the UPS, while the UPS outperforms the NUPS in terms of both $S^{G L B}$ and $S^{B H}$.

\section{Individual VR Performance}

In this subsection, we investigate the performance of each individual VR, including the price charged to them for renting

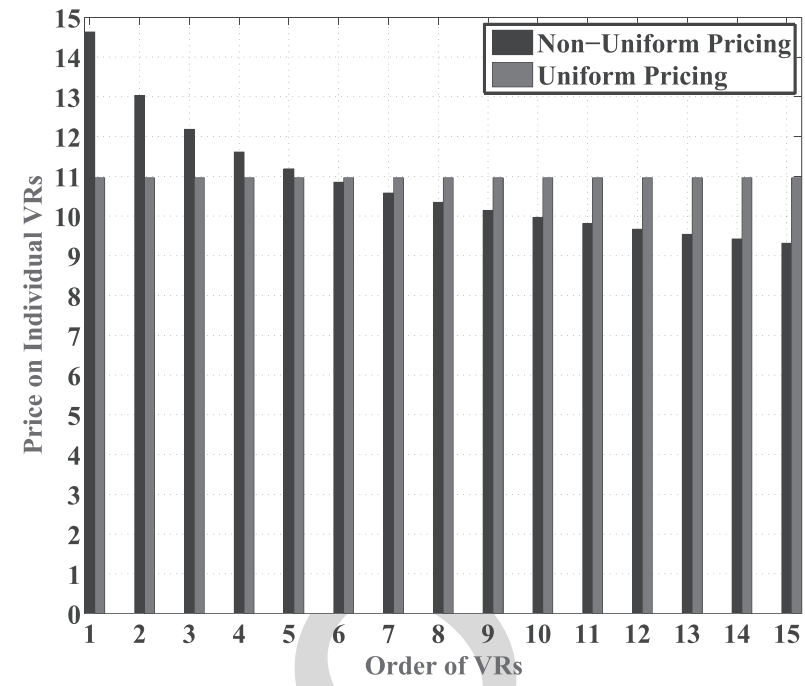

Fig. 8. Price charged on each VR for renting an SBS per month.

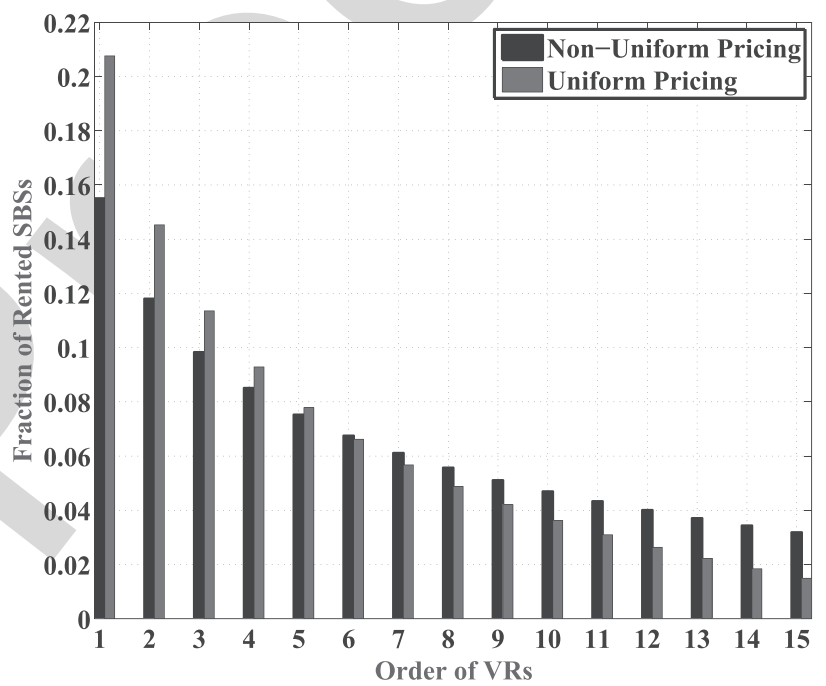

Fig. 9. The fraction of SBSs that are rented by each VR.

an SBS per month, and the fractions of the SBSs they rent from the NSP. We fix $\gamma=0.5$ and choose a large storage size of $Q=500$ for ensuring that all the VRs can be included. Fig. 8 shows the price charged to each VR for renting an SBS. The VRs are arranged according to their popularity order, ranging from $\mathcal{V}_{1}$ to $\mathcal{V}_{15}$, with $\mathcal{V}_{1}$ having the highest popularity and $\mathcal{V}_{15}$ the lowest one. We can see from the figure that in the NUPS, the price for renting an SBS is higher for the VRs having a higher popularity than those with a lower popularity. By contrast, in the UPS, this price is fixed for all the VRs. Fig. 9 shows the specific fraction of the rented SBSs at each VR. In both schemes, the VRs associated with a high popularity tend to rent more SBSs. The UPS in fact represents an instance of the water-filling algorithm. Furthermore, the UPS seems more aggressive than the NUPS, since the less popular VRs of the UPS are more difficult to rent an SBS, and thus these VRs are likely to be excluded from the game with a higher probability.

\section{CONCLUSIONS}

In this paper, we considered a commercial small-cell caching system consisting of an NSP and multiple VRs, where 
the NSP leases its SBSs to the VRs for gaining profits and for reducing the costs of back-haul channel transmissions, while the VRs, after storing popular videos to the rented SBSs, can provide faster transmissions to the MUs, hence gaining more profits. We proposed a Stackelberg game theoretic framework by viewing the SBSs as a type of resources. We first modeled the MUs and SBSs using two independent PPPs with the aid of stochastic geometry, and developed the probability expression of direct downloading. Then, based on the probability derived, we formulated a Stackelberg game for maximizing the average profit of the NSP as well as individual VRs. Next, we investigate the Stackelberg equilibrium by solving the associated nonconvex optimization problem. We considered a non-uniform pricing scheme and an uniform pricing scheme. In the former scheme, the prices charged to each VR for renting an SBS are different, while the latter imposes the same price for each VR. We proved that the non-uniform pricing scheme can effectively maximize the profit of the NSP, while the uniform one maximizes the sum profit of the NSP and the VRs. Furthermore, we derived a relationship between the optimal pricing of renting an SBS, the fraction of SBSs rented by each VR, the storage size of each SBS and the popularity of the VRs. We verified by Monte-Carlo simulations that the direct downloading probability under our PPP model is consistent with our derived results. Then we provided several numerical results for showing that the proposed schemes are effective in both pricing and SBSs allocation.

\section{APPENDIX A}

PROOF OF THEOREM 1

Recall that the SBSs allocated to the VR $\mathcal{V}_{v}$ and cache $\mathcal{G}_{f}$ are modeled as a "thinned" HPPP $\Phi_{v, f}$ having the intensity of $\frac{1}{F} \tau_{v} \lambda$. We consider a typical MU $\mathcal{M}$ who wishes to connect to the nearest $\mathrm{SBS} \mathcal{B}$ in $\Phi_{v, f}$. The event $\mathcal{E}_{v, f}$ represents that this SBS can support $\mathcal{M}$ with an SINR no lower than $\delta$, and thus $\mathcal{M}$ can obtain the desired file from the cache of $\mathcal{B}$.

We carry out the analysis on $\operatorname{Pr}\left(\mathcal{E}_{v}, f\right)$ for the typical MU $\mathcal{M}$ located at the origin. Since the network is interference dominant, we neglect the noise in the following. We denote by $z$ the distance between $\mathcal{M}$ and $\mathcal{B}$, by $x_{Z}$ the location of $\mathcal{B}$, and by $\rho\left(x_{Z}\right)$ the received SINR at $\mathcal{M}$ from $\mathcal{B}$. Then the average probability that $\mathcal{M}$ can download the desired video from $\mathcal{B}$ is

$\operatorname{Pr}\left(\rho\left(x_{Z}\right) \geq \delta\right)$

$=\int_{0}^{\infty} \operatorname{Pr}\left(\frac{h_{x_{Z} z^{-\alpha}}}{\sum_{x \in \Phi \backslash\left\{x_{Z}\right\}} h_{x}\|x\|^{-\alpha}} \geq \delta \mid z\right) f_{Z}(z) \mathrm{d} z$

$=\int_{0}^{\infty} \operatorname{Pr}\left(h_{x_{Z}} \geq \frac{\delta\left(\sum_{x \in \Phi \backslash\left\{x_{Z}\right\}} h_{x}\|x\|^{-\alpha}\right)}{z^{-\alpha}} \mid z\right)$

$$
\begin{gathered}
2 \pi \frac{1}{F} \tau_{v} \lambda z \exp \left(-\pi \frac{1}{F} \tau_{v} \lambda z^{2}\right) \mathrm{d} z \\
=\int_{0}^{\infty} \mathbb{E}_{I}\left(\exp \left(-z^{\alpha} \delta I\right)\right) 2 \pi \frac{1}{F} \tau_{v} \lambda z \exp \left(-\pi \frac{1}{F} \tau_{v} \lambda z^{2}\right) \mathrm{d} z,
\end{gathered}
$$

where we have $I \triangleq \sum_{x \in \Phi \backslash\left\{x_{Z}\right\}} h_{x}\|x\|^{-\alpha}$, and the PDF of $z$, i.e., $\quad 907$ $f_{Z}(z)$, is derived by the null probability of the HPPP $\Phi_{v, f} \quad 908$ with the intensity of $\frac{1}{F} \tau_{v} \lambda$. More specifically in $\Phi_{v, f}$, since $\quad 909$ the number of the SBSs $k$ in an area of $A$ follows the Poisson distribution, the probability of the event that there is no SBS in the area with the radius of $z$ can be calculated as [17]

$$
\operatorname{Pr}\left(k=0 \mid A=\pi z^{2}\right)=e^{-A \frac{1}{F} \tau_{v} \lambda} \frac{\left(A \frac{1}{F} \tau_{v} \lambda\right)^{k}}{k !}=e^{-\pi z^{2} \frac{1}{F} \tau_{v} \lambda} .
$$

By using the above expression, we arrive at $f_{Z}(z)=$ $2 \pi \frac{1}{F} \tau_{v} \lambda z \exp \left(-\pi \frac{1}{F} \tau_{v} \lambda z^{2}\right)$. Note that the interference $I$ consists of $I_{1}$ and $I_{2}$, where $I_{1}$ emanates from the SBSs in $\Phi$ excluding $\Phi_{v, f}$, while $I_{2}$ is from the SBSs in $\Phi_{v, f}$ excluding $\mathcal{B}$. The SBSs contributing to $I_{1}$, denoted by $\Phi_{\bar{v}, f}$, have the intensity of $\left(1-\frac{1}{F} \tau_{v}\right) \lambda$, while those contributing to $I_{2}$ have the intensity of $\frac{1}{F} \tau_{v} \lambda$.

Correspondingly, the calculation of $\mathbb{E}_{I}\left(\exp \left(-z^{\alpha} \delta I\right)\right)$ will be split into the product of two expectations over $I_{1}$ and $I_{2}$. The expectation over $I_{1}$ is calculated as

$$
\begin{aligned}
& \mathbb{E}_{I_{1}}\left(\exp \left(-z^{\alpha} \delta I_{1}\right)\right) \\
& \stackrel{(a)}{=} \mathbb{E}_{\Phi_{\overline{v, f}}}\left(\prod_{x \in \Phi_{\bar{v}}} \int_{0}^{\infty} \exp \left(-z^{\alpha} \delta h_{x}\|x\|^{-\alpha}\right) \exp \left(-h_{x}\right) \mathrm{d} h_{x}\right) \\
& \stackrel{(b)}{=} \exp \left(-\left(1-\frac{1}{F} \tau_{v}\right) \lambda \int_{\mathbb{R}^{2}}\left(1-\frac{1}{1+z^{\alpha} \delta\left\|x_{k}\right\|^{-\alpha}}\right) \mathrm{d} x_{k}\right) \\
& =\exp \left(-2 \pi\left(1-\frac{1}{F} \tau_{v}\right) \lambda \frac{1}{\alpha} z^{2} \delta^{\frac{2}{\alpha}} B\left(\frac{2}{\alpha}, 1-\frac{2}{\alpha}\right)\right) \\
& =\exp \left(-\pi\left(1-\frac{1}{F} \tau_{v}\right) \lambda C(\delta, \alpha) z^{2}\right)
\end{aligned}
$$

where $(a)$ is based on the independence of channel fading, while $(b)$ follows from $\mathbb{E}\left(\prod_{x} u(x)\right)=$ $\exp \left(-\lambda \int_{\mathbb{R}^{2}}(1-u(x)) \mathrm{d} x\right)$, where $x \in \Phi$ and $\Phi$ is an PPP in $\mathbb{R}^{2}$ with the intensity $\lambda[24]$, and $C(\delta, \alpha)$ has been defined as $\frac{2}{\alpha} \delta^{\frac{2}{\alpha}} B\left(\frac{2}{\alpha}, 1-\frac{2}{\alpha}\right)$.

The expectation over $I_{2}$ has to take into account $z$ as the distance from the nearest interfering SBS. Then we have

$$
\begin{aligned}
& \mathbb{E}_{I_{2}}\left(\exp \left(-z^{\alpha} \delta I_{2}\right)\right) \\
& =\exp \left(-\frac{1}{F} \tau_{v} \lambda 2 \pi \int_{z}^{\infty}\left(1-\frac{1}{1+z^{\alpha} \delta r^{-\alpha}}\right) r \mathrm{~d} r\right) \\
& \stackrel{(a)}{=} \exp \left(-\frac{1}{F} \tau_{v} \lambda \pi \delta^{\frac{2}{\alpha}} z^{2} \frac{2}{\alpha} \int_{\delta^{-1}}^{\infty} \frac{\kappa^{\frac{2}{\alpha}-1}}{1+\kappa} \mathrm{d} x\right) \\
& \stackrel{(b)}{=} \exp \left(-\frac{1}{F} \tau_{v} \lambda \pi \delta z^{2} \frac{2}{\alpha-2}{ }_{2} F_{1}\left(1,1-\frac{2}{\alpha} ; 2-\frac{2}{\alpha} ;-\delta\right)\right)
\end{aligned}
$$

where $(a)$ defines $\kappa \triangleq \delta^{-1} z^{-\alpha} r^{\alpha}$, and ${ }_{2} F_{1}(\cdot)$ in $(b)$ is the hypergeometric function. As we defined $A(\delta, \alpha)=\frac{2 \delta}{\alpha-2} \quad 2 F_{1}\left(1,1-\frac{2}{\alpha} ; 2-\frac{2}{\alpha} ;-\delta\right)$, by

915 916 917 918 919 920 921 922 923 924

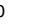

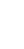

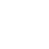


substituting (49) and (50) into (47), we have

$$
\begin{aligned}
& \operatorname{Pr}\left(\rho\left(x_{Z}\right) \geq \delta\right) \\
& =\int_{0}^{\infty} \exp \left(-\pi\left(1-\frac{1}{F} \tau_{v}\right) \lambda C(\delta, \alpha) z^{2}\right) \\
& \quad \exp \left(-\pi \frac{1}{F} \tau_{v} \lambda z^{2} A(\delta, \alpha)\right) 2 \pi \frac{1}{F} \tau_{v} \lambda z \exp \left(-\pi \frac{1}{F} \tau_{v} \lambda z^{2}\right) \mathrm{d} z \\
& =\frac{\frac{1}{F} \tau_{v}}{C(\delta, \alpha)\left(1-\frac{1}{F} \tau_{v}\right)+A(\delta, \alpha) \frac{1}{F} \tau_{v}+\frac{1}{F} \tau_{v}}
\end{aligned}
$$

This completes the proof.

\section{APPENDiX B}

\section{ProOf OF LEMMA 2}

By applying Lagrangian multipliers to the objective function, we have

$L(\mathbf{s}, \mu, \boldsymbol{v})$

$=\sum_{j=1}^{V} s_{j}+\mu\left(\sum_{j=1}^{V} \sqrt{\frac{\Gamma_{j}}{s_{j}}}-(V \Lambda+\Theta) \sqrt{\frac{\lambda}{\Lambda s^{b h}}}\right)-\sum_{j=1}^{V} v_{j} s_{j}$,

where $\mu$ and $v_{j}$ are non-negative multipliers associated with the constraints $\sum_{j=1}^{V} \sqrt{\frac{\Gamma_{j}}{s_{j}}}-(V \Lambda+\Theta) \sqrt{\frac{\lambda}{\Lambda s^{b h}}} \leq 0$ and $s_{j} \geq 0$, respectively. Then the KKT conditions can be written as

$$
\begin{aligned}
\frac{\partial L(\mathbf{s}, \mu, \boldsymbol{v})}{\partial s_{j}} & =0, \quad \forall j=1, \cdots, V, \\
\mu\left(\sum_{j=1}^{V} \sqrt{\frac{\Gamma_{j}}{s_{j}}}-(V \Lambda+\Theta) \sqrt{\frac{\lambda}{\Lambda s^{b h}}}\right) & =0, \text { and } v_{j} s_{j}=0, \quad \forall j .
\end{aligned}
$$

From the first line of Eq. (53), we have

$$
s_{j}=\sqrt[3]{\frac{\mu^{2} \Gamma_{j}}{4\left(1-v_{j}\right)^{2}}} .
$$

Obviously, we have $s_{j} \neq 0, \forall j$, otherwise the constraint $\sum_{j=1}^{V} \sqrt{\frac{\Gamma_{j}}{s_{j}}}-(V \Lambda+\Theta) \sqrt{\frac{\lambda}{\Lambda s^{b h}}} \leq 0$ cannot be satisfied. Thus, we have $v_{j}=0, \forall j$. Furthermore, we have $\mu \neq 0$ according to Eq. (54) since $s_{j}$ is non-zero. This means that $\sum_{j=1}^{V} \sqrt{\frac{\Gamma_{j}}{s_{j}}}-(V \Lambda+\Theta) \sqrt{\frac{\lambda}{\Lambda s^{b h}}}=0$.

By substituting Eq. (54) into this constraint, we have

$$
\sqrt[3]{\mu}=\frac{\sqrt{\Lambda s^{b h}} \sum_{j=1}^{V} \sqrt[3]{2 \Gamma_{j}}}{\sqrt{\lambda}(V \Lambda+\Theta)}
$$

Then it follows that

$$
s_{j}=\frac{\Lambda s^{b h}\left(\sum_{v=1}^{V} \sqrt[3]{\Gamma_{v}}\right)^{2} \sqrt[3]{\Gamma_{j}}}{\lambda(V \Lambda+\Theta)^{2}} .
$$

This completes the proof.

APPENDIX C Proof of THEOREM 2

As discussed in Eq. (23) and Eq. (24), we have proved that $Q>\frac{N C(\delta, \alpha)\left(\sum_{j=1}^{V} \sqrt[3]{\frac{q_{j}}{q_{V}}}-V\right)}{A(\delta, \alpha)-C(\delta, \alpha)+1}$ is a sufficient condition for the optimal solution in Eq. (22). In other words, as long as $Q$ is satisfied, we have the conclusion that the solution in Eq. (22) is optimal and $\xi_{p}=1, \forall v$. generality, we assume that

$$
\begin{gathered}
\frac{N C(\delta, \alpha)\left(\sum_{j=1}^{V-1} \sqrt[3]{\frac{q_{j}}{q_{V-1}}}-V+1\right)}{A(\delta, \alpha)-C(\delta, \alpha)+1}<Q \\
\leq \frac{N C(\delta, \alpha)\left(\sum_{j=1}^{V} \sqrt[3]{\frac{q_{j}}{q_{V}}}-V\right)}{A(\delta, \alpha)-C(\delta, \alpha)+1} .
\end{gathered}
$$

This leads to $s_{V} \geq \frac{\Gamma_{v} s^{b h}}{\Lambda \lambda}$, and the VR $\mathcal{V}_{V}$ will be excluded from the game. In this case, we have $\xi_{j}=1, j=1, \cdots, V-1$, and Problem 4 will be rewritten as follows.

Problem 9: We rewrite Problem 4 as

$$
\begin{aligned}
& \min _{\mathbf{s} \geq \mathbf{0}} \sum_{j=1}^{V-1} s_{j}, \\
& \text { s.t. } \sum_{j=1}^{V-1} \sqrt{\frac{\Gamma_{j}}{s_{j}}} \leq((V-1) \Lambda+\Theta) \sqrt{\frac{\lambda}{\Lambda s^{b h}}} .
\end{aligned}
$$

Similar to the proof of Lemma 2, and combined with the 993 constraint of $Q$ in Eq. (57), the optimal solution of Problem 9 is given by

$$
\hat{s}_{v}= \begin{cases}\frac{\Lambda s^{b h}\left(\sum_{j=1}^{V-1} \sqrt[3]{\Gamma_{j}}\right)^{2} \sqrt[3]{\Gamma_{v}}}{\lambda((V-1) \Lambda+\Theta)^{2}}, & v=1, \cdots, V-1, \\ \infty, & v=V .\end{cases}
$$

We can see that the optimal solution given in Eq. (59) contradicts to the optimal solution of Problem 4 given in Eq. (22). Hence, $Q>\frac{N C(\delta, \alpha)\left(\sum_{j=1}^{V} \sqrt[3]{\frac{q_{j}}{q_{V}}}-V\right)}{A(\delta, \alpha)-C(\delta, \alpha)+1}$ is a necessary condition for finding the optimal solution in Eq. (22). This completes the proof.

\section{APPENDIX D}

PROOF OF LEMMA 3

Consider $v_{1}, v_{2}=1, \cdots, V$ and $v_{1}=v_{2}+1$. Then we prove that $U_{v_{1}}>U_{v_{2}}$. We have

$$
\begin{aligned}
& U_{v_{1}}=\frac{N C(\delta, \alpha)\left(\sum_{j=1}^{v_{1}} \sqrt[3]{\frac{q_{j}}{q_{v_{1}}}}-v_{1}\right)}{A(\delta, \alpha)-C(\delta, \alpha)+1} \\
& =\frac{N C(\delta, \alpha)\left(\sum_{j=1}^{v_{2}} \sqrt[3]{\frac{q_{j}}{q_{v_{1}}}}-v_{2}+\sum_{j=v_{2}+1}^{v_{1}} \sqrt[3]{\frac{q_{j}}{q_{v_{1}}}}-\left(v_{1}-v_{2}\right)\right)}{A(\delta, \alpha)-C(\delta, \alpha)+1} \\
& =\frac{N C(\delta, \alpha)\left(\sum_{j=1}^{v_{2}} \sqrt[3]{\frac{q_{j}}{q_{v_{1}}}}-v_{2}\right)}{A(\delta, \alpha)-C(\delta, \alpha)+1} \\
& \stackrel{\text { (a) }}{>} \frac{N C(\delta, \alpha)\left(\sum_{j=1}^{v_{2}} \sqrt[3]{\frac{q_{j}}{q_{v_{2}}}}-v_{2}\right)}{A(\delta, \alpha)-C(\delta, \alpha)+1}=U_{v_{2}},
\end{aligned}
$$

992 
where $(a)$ comes from the fact that $q_{v_{1}}<q_{v_{2}}$. This completes the proof.

\section{APPENDIX E \\ PROOF OF LEMMA 4}

It is plausible that if $\mathcal{L}$ can only keep at most $v$ VRs, it has to retain the $v$ most popular VRs to maximize its profit. Let us now prove that if $\mathcal{L}$ keeps $(v+w)$ VRs, $w=1, \cdots, V-v$, in the game, it cannot achieve the optimal solution for $U_{v}<Q \leq U_{v+1}$.

Problem 10: In the case that $\mathcal{L}$ keeps $(v+w)$ VRs, we have the optimization problem of

2

$$
\begin{aligned}
& \min _{\mathbf{s} \succeq \mathbf{0}} \sum_{j=1}^{v+w} s_{j}, \\
& \text { s.t. } \sum_{j=1}^{v+w} \sqrt{\frac{\Gamma_{j}}{s_{j}}} \leq((v+w) \Lambda+\Theta) \sqrt{\frac{\lambda}{\Lambda s^{b h}}} .
\end{aligned}
$$

Similar to the proof of Theorem 2, we obtain that $Q>$ $\frac{N C(\delta, \alpha)\left(\sum_{j=1}^{v+w} \sqrt[3]{\frac{q_{j}}{q_{v+w}}}-(v+w)\right)}{A(\delta, \alpha)-C(\delta, \alpha)+1}=U_{v+w}$ is the necessary condition for the $(v+w)$ VRs to participate in the game. This contradicts to the premise $U_{v}<Q \leq U_{v+1}$, since we have $Q>U_{v+1}$ according to Lemma 3. Let us now consider the cases of $w^{\prime}=0,-1, \cdots, 1-v$. To ensure there are $\left(v+w^{\prime}\right)$ VRs in the game, $Q$ has to satisfy the condition that $Q>U_{v+w^{\prime}}$. Since $Q>U_{v} \geq U_{v+w^{\prime}}$, this implies that given $\left(v+w^{\prime}\right)$ VRs in the game, the NSP can achieve an optimal solution. This completes the proof.

\section{REFERENCES}

[1] N. Golrezaei, A. F. Molisch, A. G. Dimakis, and G. Caire, "Femtocaching and device-to-device collaboration: A new architecture for wireless video distribution," IEEE Commun. Mag., vol. 51, no. 4, pp. 142-149, Apr. 2013.

[2] X. Wang, M. Chen, T. Taleb, A. Ksentini, and V. C. M. Leung, "Cache in the air: Exploiting content caching and delivery techniques for $5 \mathrm{G}$ systems," IEEE Commun. Mag., vol. 52, no. 2, pp. 131-139, Feb. 2014.

[3] M. A. Maddah-Ali and U. Niesen, "Decentralized coded caching attains order-optimal memory-rate tradeoff," in Proc. 51st Annu. Allerton Conf. Commun., Control, Comput. (Allerton), Oct. 2013, pp. 421-427.

[4] N. Golrezaei, P. Mansourifard, A. F. Molisch, and A. G. Dimakis, "Basestation assisted device-to-device communications for high-throughput wireless video networks," IEEE Trans. Wireless Commun., vol. 13, no. 7, pp. 3665-3676, Jul. 2014.

[5] M. Ji, G. Caire, and A. F. Molisch. (May 2013). "Wireless device-todevice caching networks: Basic principles and system performance." [Online]. Available: http://arxiv.org/abs/1305.5216

[6] M. Ji, G. Caire, and A. F. Molisch, "Optimal throughput-outage tradeoff in wireless one-hop caching networks," in Proc. IEEE Int. Symp. Inf. Theory (ISIT), Jul. 2013, pp. 1461-1465.

[7] P. Gupta and P. R. Kumar, "The capacity of wireless networks," IEEE Trans. Inf. Theory, vol. 46, no. 2, pp. 388-404, Mar. 2000.

[8] F. Boccardi, R. W. Heath, A. Lozano, T. L. Marzetta, and P. Popovski, "Five disruptive technology directions for 5G," IEEE Commun. Mag., vol. 52, no. 2, pp. 74-80, Feb. 2014

[9] A. Damnjanovic et al., "A survey on 3GPP heterogeneous networks," IEEE Wireless Commun., vol. 18, no. 3, pp. 10-21, Jun. 2011.

[10] J. Akhtman and L. Hanzo, "Heterogeneous networking: An enabling paradigm for ubiquitous wireless communications," Proc. IEEE, vol. 98, no. 2, pp. 135-138, Feb. 2010.
[11] S. Bayat, R. H. Y. Louie, Z. Han, B. Vucetic, and Y. Li, "Distributed user association and femtocell allocation in heterogeneous wireless networks," IEEE Trans. Commun., vol. 62, no. 8, pp. 3027-3043, Aug. 2014.

[12] M. Mirahmadi, A. Al-Dweik, and A. Shami, "Interference modeling and performance evaluation of heterogeneous cellular networks," IEEE Trans. Commun., vol. 62, no. 6, pp. 2132-2144, Jun. 2014.

[13] A. K. Gupta, H. S. Dhillon, S. Vishwanath, and J. G. Andrews, "Downlink multi-antenna heterogeneous cellular network with load balancing," IEEE Trans. Commun., vol. 62, no. 11, pp. 4052-4067, Nov. 2014.

[14] M. Liebsch, S. Schmid, and J. Awano, "Reducing backhaul costs for mobile content delivery-An analytical study," in Proc. IEEE Int. Conf. Commun. (ICC), Jun. 2012, pp. 2895-2900.

[15] K. Shanmugam, N. Golrezaei, A. G. Dimakis, A. F. Molisch, and G. Caire, "FemtoCaching: Wireless content delivery through distributed caching helpers," IEEE Trans. Inf. Theory, vol. 59, no. 12, pp. 8402-8413, Dec. 2013.

[16] E. Baştuğ, M. Bennis, and M. Debbah, "Cache-enabled small cell networks: Modeling and tradeoffs," in Proc. 11th Int. Symp. Wireles. Commun. Syst. (ISWCS), Aug. 2014, pp. 649-653.

[17] D. Stoyan, W. S. Kendall, and M. Mecke, Stochastic Geometry and Its Applications. 2nd ed. New York, NY, USA: Wiley, 2003.

[18] M. Haenggi, J. G. Andrews, F. Baccelli, O. Dousse, and M. Franceschetti, "Stochastic geometry and random graphs for the analysis and design of wireless networks," IEEE J. Sel. Areas Commun. vol. 27, no. 7, pp. 1029-1046, Sep. 2009.

[19] G. Vazquez-Vilar, C. Mosquera, and S. K. Jayaweera, "Primary user enters the game: Performance of dynamic spectrum leasing in cognitive radio networks," IEEE Trans. Wireless Commun., vol. 9, no. 12, pp. 3625-3629, Dec. 2010.

[20] X. Kang, R. Zhang, and M. Motani, "Price-based resource allocation for spectrum-sharing femtocell networks: A Stackelberg game approach," IEEE J. Sel. Areas Commun., vol. 30, no. 3, pp. 538-549, Apr. 2012.

[21] D. Niyato and E. Hossain, "Competitive spectrum sharing in cognitive radio networks: A dynamic game approach," IEEE Trans. Wireless Commun., vol. 7, no. 7, pp. 2651-2660, Jul. 2008

[22] D. Niyato, E. Hossain, and Z. Han, "Dynamics of multiple-seller and multiple-buyer spectrum trading in cognitive radio networks: A gametheoretic modeling approach," IEEE Trans. Mobile Comput., vol. 8, no. 8, pp. 1009-1022, Aug. 2009.

[23] D. Fudenberg and J. Tirole, Game Theory. Cambridge, MA, USA: MIT Press, 1993.

[24] D. J. Daley and D. Vere-Jones, An Introduction to the Theory of Point Processes: Elementary Theory and Methods, vol. 1. Springer, 1996.

[25] M. Cha, H. Kwak, P. Rodriguez, Y.-Y. Ahn, and S. Moon, "iTube, You Tube, everybody tubes: Analyzing the world's largest user generated content video system," in Proc. 7th ACM SIGCOMM Conf. Internet Meas., 2007, pp. 1-14.

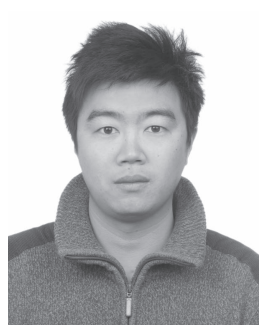

Jun Li (M'09) received the Ph.D. degree in electronics engineering from Shanghai Jiao Tong University, Shanghai, China, in 2009. In 2009, he was with the Department of Research and Innovation, Alcatel Lucent Shanghai Bell, as a Research Scientist. From 2009 to 2012, he was a Post-Doctoral Fellow with the School of Electrical Engineering and Telecommunications, University of New South Wales, Australia. From 2012 to 2015, he was a Research Fellow with the School of Electrical Engineering, The University of Sydney, Australia. Since 2015, he has been a Professor with the School of Electronic and Optical Engineering, Nanjing University of Science and Technology, Nanjing, China. His research interests include network information theory, channel coding theory, wireless network coding, and cooperative communications. 

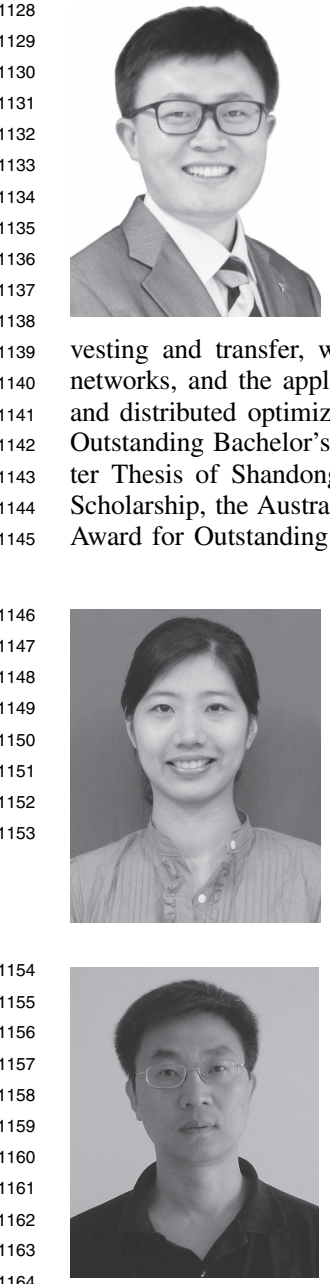

He (Henry) Chen (S'10-M'16) received the B.E. degree in communication engineering and the M.E. degree in communication and information systems from Shandong University, Jinan, China, in 2008 and 2011, respectively, and the Ph.D. degree in electrical engineering from The University of Sydney, Sydney, Australia, in 2015. He is currently a Research Fellow with the School of Electrical and Information Engineering, The University of Sydney. His current research interests include millimeterwave wireless communications, wireless energy harvesting and transfer, wireless network virtualization, cooperative and relay networks, and the applications of game theory, variational inequality theory, and distributed optimization theory in these areas. He was a recipient of the Outstanding Bachelor's Thesis of Shandong University, the Outstanding Master Thesis of Shandong Province, the International Post-Graduate Research Scholarship, the Australian Postgraduate Award, and the Chinese Government Award for Outstanding Self-Financed Students Abroad.

Youjia Chen received the B.S. and M.S. degrees in communication engineering from Nanjing University, Nanjing, China, in 2005 and 2008, respectively. She is currently pursuing the Ph.D. degree in wireless engineering with The University of Sydney, Sydney, Australia. Her current research interests include resource management, load balancing, and caching strategy in heterogeneous cellular networks. modulation, MIMO, OFDMA, SCFDMA, radio resource management, cooperative communications, small-cell networks, and 5G cellular systems.

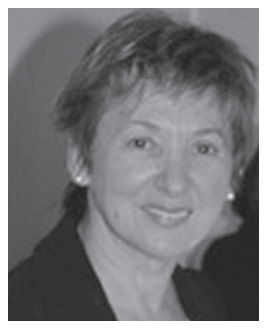

Branka Vucetic (M'83-SM'00-F'03) has held various research and academic positions in Yugoslavia, Australia, U.K., and China. During her career, she co-authored 4 books and more than 400 papers in telecommunications journals and conference proceedings. She currently holds the Peter Nicol Russe Chair of Telecommunications Engineering with The University of Sydney. Her research interest include wireless communications, coding, digita communication theory, and machine-to-machine communications.
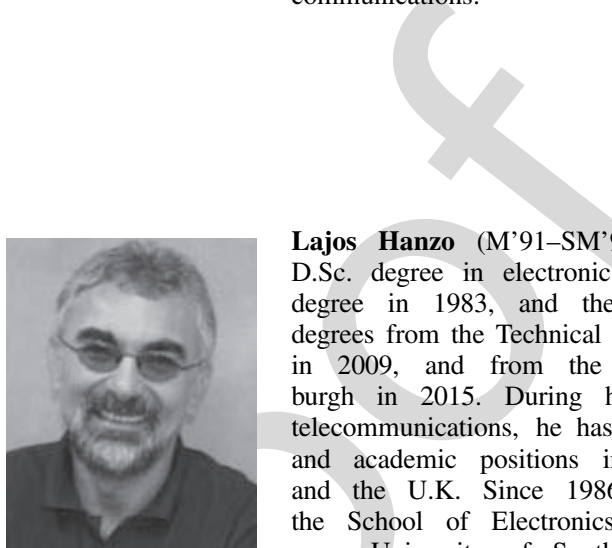

Lajos Hanzo (M'91-SM'92-F'04) received the D.Sc. degree in electronics in 1976, the Ph.D. degree in 1983, and the Honorary Doctorate degrees from the Technical University of Budapes in 2009, and from the University of Edinburgh in 2015. During his 38-year career in telecommunications, he has held various research and academic positions in Hungary, Germany, and the U.K. Since 1986, he has been with the School of Electronics and Computer Science, University of Southampton, U.K., where he holds the Chair in Telecommunications. He has successfully supervised about 100 Ph.D. students, co-authored 20 John Wiley/IEEE Press books on mobile radio communications totaling in excess of 10000 pages, published over 1500 research entries at the IEEE Xplore, acted both as a TPC and General Chair of the IEEE conferences, presented keynote lectures, and has received a number of distinctions. He is currently directing 60 strong academic research teams, working on a range of research projects in the field of wireless multimedia communications sponsored by industry, the Engineering and Physical Sciences Research Council, U.K., the European Research Council's Advanced Fellow Grant, and the Royal Society's Wolfson Research Merit Award. He has 24000 citations. $\mathrm{He}$ is an enthusiastic supporter of industrial and academic liaison. He offers a range of industrial courses. He is also a Governor of the IEEE VTS. From 2008 to 2012, he was the Editor-in-Chief of the IEEE Press and a Chaired Professor with Tsinghua University, Beijing. He is a fellow of REng, IET, and EURASIP.
1179 AQ:6 


\section{AUTHOR QUERIES}

\section{AUTHOR PLEASE ANSWER ALL QUERIES}

PLEASE NOTE: We cannot accept new source files as corrections for your paper. If possible, please annotate the PDF proof we have sent you with your corrections and upload it via the Author Gateway. Alternatively, you may send us your corrections in list format. You may also upload revised graphics via the Author Gateway.

AQ:1 = Please be advised that per instructions from the Communications Society this proof was formatted in Times Roman font and therefore some of the fonts will appear different from the fonts in your originally submitted manuscript. For instance, the math calligraphy font may appear different due to usage of the usepackage[mathcal] euscript. We are no longer permitted to use Computer Modern fonts.

AQ:2 = Please confirm the postal codes for "The University of Sydney and University of Southampton."

AQ:3 = Note that if you require corrections/changes to tables or figures, you must supply the revised files, as these items are not edited for you.

AQ:4 = Please provide the publisher location for ref. [24].

AQ:5 = Please confirm the article title for ref. [25].

AQ:6 = Please confirm whether the edits made in the sentence "Lajos Hanzo received $\ldots$ of Edinburgh in 2015." are OK. 